\title{
CuI-Catalyzed Decarboxylative Thiolation of Propargylic Cyclic Carbonates/Carbamates to Access Allenyl Thioethers
}

Wen-Ya Lu, ${ }^{+, \neq, \S}$ Yong You, ${ }^{\ddagger, *}$ Ting-Ting Li, ${ }^{+, \neq, \S}$ Zhen-Hua Wang, ${ }^{\ddagger}$ Jian-Qiang Zhao, ${ }^{\ddagger}$ and WeiCheng Yuan ${ }^{+, \neq, \S, *}$

${ }^{+}$National Engineering Research Center of Chiral Drugs, Chengdu Institute of Organic Chemistry, Chinese Academy of Sciences, Chengdu, 610041, China

${ }^{\ddagger}$ Institute for Advanced Study, Chengdu University, Chengdu 610106, China

${ }^{\S}$ University of Chinese Academy of Sciences, Beijing, 100049, China

yuanwc@cioc.ac.cn

youyong@cdu.edu.cn

\section{Supporting Information}

\section{Contents}

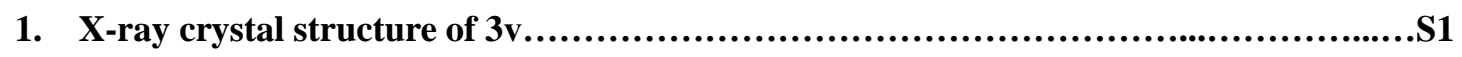

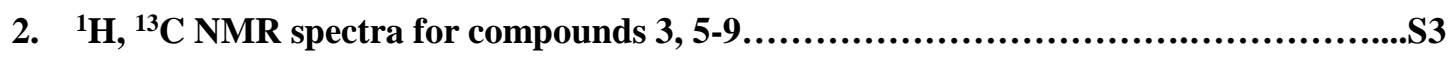




\section{X-ray crystal structure of $3 v$}

Single crystals of compound $\mathbf{3 v}$ were prepared through dissolving the sample in mixture solvent of EtOH/DCM (10/1) at room temperature and crystalizing by slow evaporation of solvent. A suitable crystal was selected for structure determination on an Xcalibur, Eos, Gemini diffractometer. The crystal was kept at 293(2) K during data collection. Using Olex $2^{[1]}$, the structure was solved with the ShelXS ${ }^{[2]}$ structure solution program using Direct Methods and refined with the ShelXL ${ }^{[3]}$ refinement package using Least Squares minimisation.
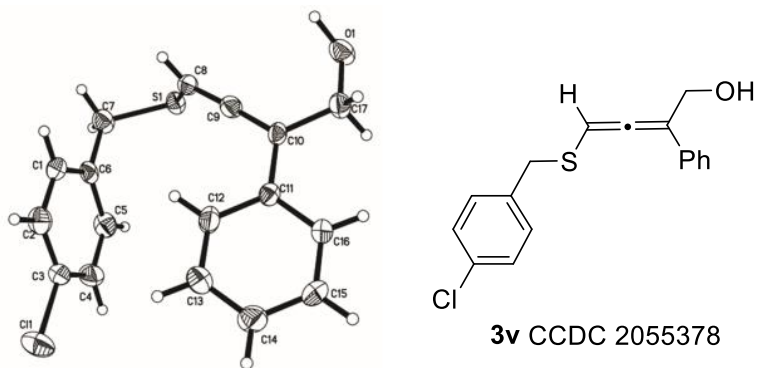

ORTEP of 3v (at $50 \%$ level)

\begin{tabular}{|l|l|}
\hline Identification code & $\mathbf{3 v}$ \\
\hline Empirical formula & $\mathrm{C}_{17} \mathrm{H}_{15} \mathrm{ClOS}$ \\
\hline Formula weight & 302.80 \\
\hline Temperature/K & $293(2)$ \\
\hline Crystal system & monoclinic \\
\hline Space group & $\mathrm{P} 2{ }_{1} / \mathrm{n}$ \\
\hline $\mathrm{a} / \AA$ & $7.9734(4)$ \\
\hline $\mathrm{b} / \AA$ & $5.4274(3)$ \\
\hline $\mathrm{c} / \AA$ & $34.8670(15)$ \\
\hline$\alpha /$ & 90 \\
\hline$\beta /$ & $92.539(4)$ \\
\hline$\gamma /$ & 90 \\
\hline Volume/ $\AA^{3}$ & $1507.39(13)$ \\
\hline $\mathrm{Z}$ & 4 \\
\hline$\rho_{\text {calcg/cm }}^{3}$ & 1.334 \\
\hline$\mu / \mathrm{mm}^{-1}$ & 3.463 \\
\hline & \\
\hline
\end{tabular}




\begin{tabular}{|l|l|}
\hline $\mathrm{F}(000)$ & 632.0 \\
\hline Crystal size/mm ${ }^{3}$ & $0.21 \times 0.1 \times 0.05$ \\
\hline Radiation & $\mathrm{CuK} \alpha(\lambda=1.54184)$ \\
\hline $2 \Theta$ range for data collection/ & 10.158 to 134.23 \\
\hline Index ranges & $-9 \leq \mathrm{h} \leq 9,-6 \leq \mathrm{k} \leq 6,-2 \leq 1 \leq 41$ \\
\hline Reflections collected & 2697 \\
\hline Independent reflections & $2697\left[\mathrm{R}_{\text {int }}=?, \mathrm{R}_{\text {sigma }}=0.0519\right]$ \\
\hline Data/restraints/parameters & $2697 / 1 / 186$ \\
\hline Goodness-of-fit on $\mathrm{F}^{2}$ & 1.098 \\
\hline Final $\mathrm{R}$ indexes [I $>=2 \sigma(\mathrm{I})]$ & $\mathrm{R}_{1}=0.0962, \mathrm{wR}_{2}=0.2523$ \\
\hline Final $\mathrm{R}$ indexes [all data] & $\mathrm{R}_{1}=0.1079, \mathrm{wR}_{2}=0.2596$ \\
\hline Largest diff. peak/hole / e $\AA^{-3}$ & $0.43 /-0.39$ \\
\hline
\end{tabular}

[1] Dolomanov, O. V.; Bourhis, L. J.; Gildea, R. J, Howard, J. A. K.; Puschmann, H. J. Appl.

Cryst.2009, 42, 339-341.

[2] Sheldrick, G. M. Acta Cryst.2008, A64, 112-122.

[3] Sheldrick, G. M. Acta Cryst.2015, C71, 3-8. 
2. ${ }^{1} \mathrm{H},{ }^{13} \mathrm{C}$ NMR spectra for compounds $3,5-9$

${ }^{1} \mathrm{H}$ NMR (300 MHz, DMSO- $\left.d_{6}\right)$ of $\mathbf{3 a}$

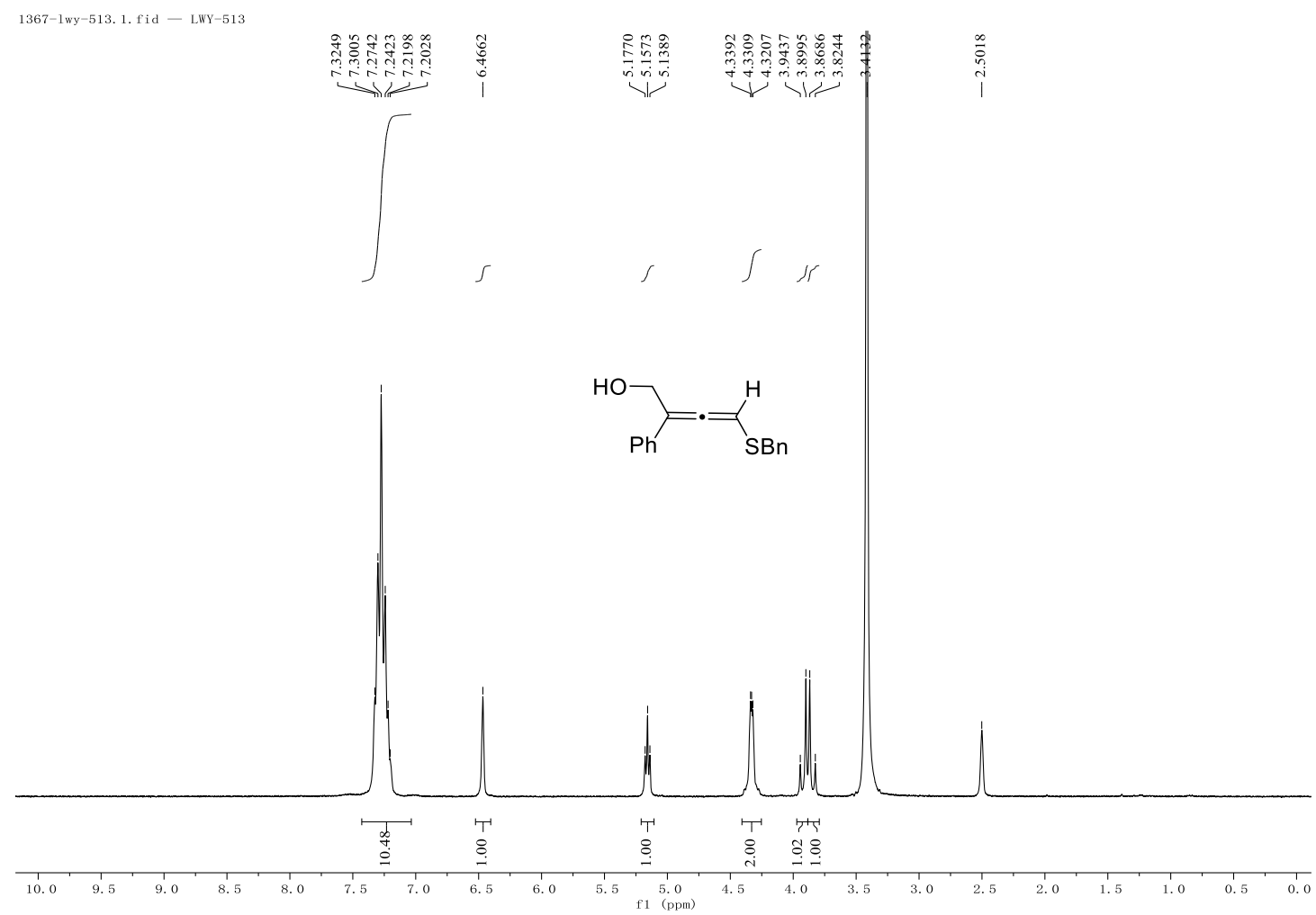

${ }^{13} \mathrm{C}\left\{{ }^{1} \mathrm{H}\right\}$ NMR (151 MHz, DMSO- $\left.d_{6}\right)$ of $\mathbf{3 a}$

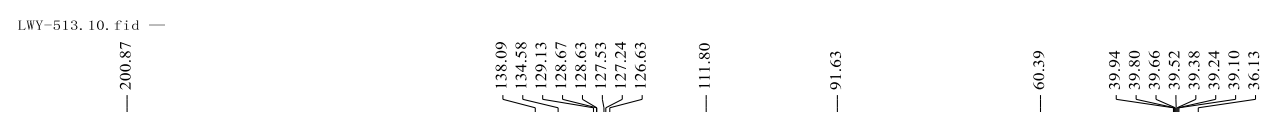
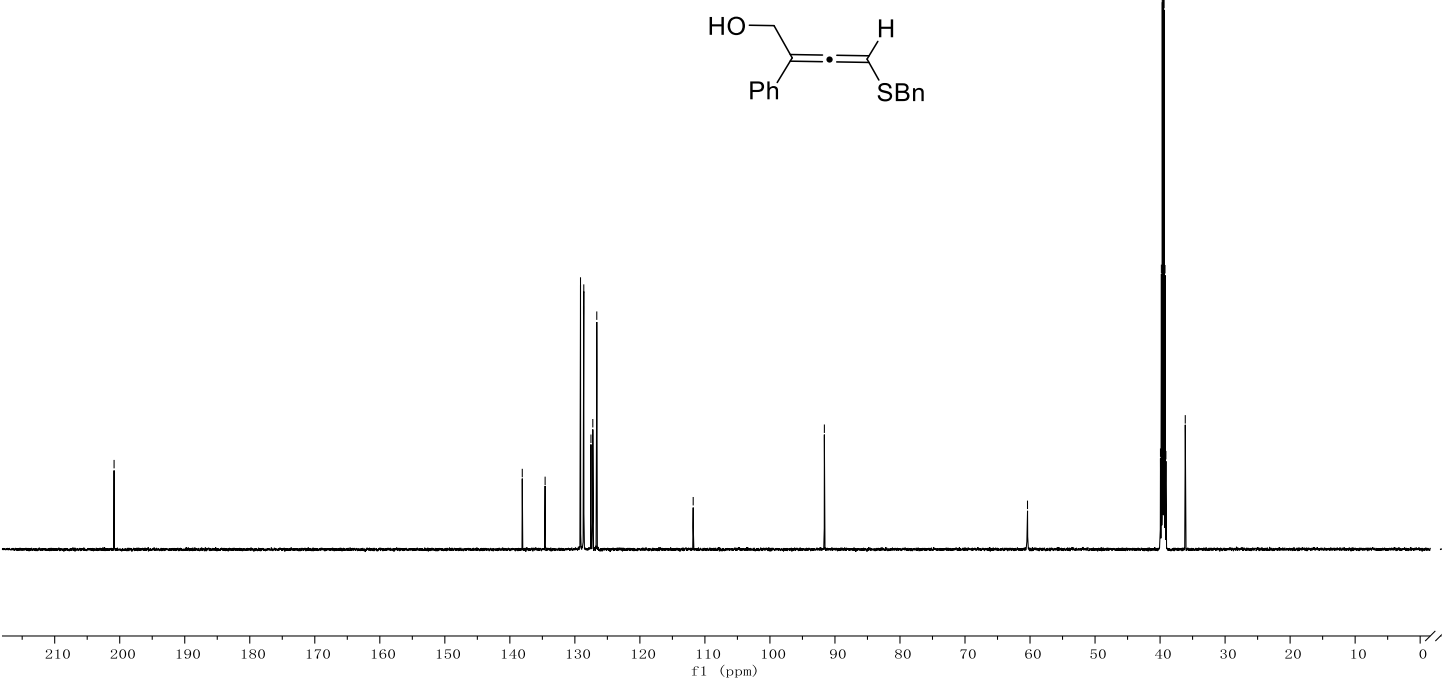
${ }^{1} \mathrm{H}$ NMR $\left(300 \mathrm{MHz}, \mathrm{DMSO}-d_{6}\right)$ of $\mathbf{3 b}$

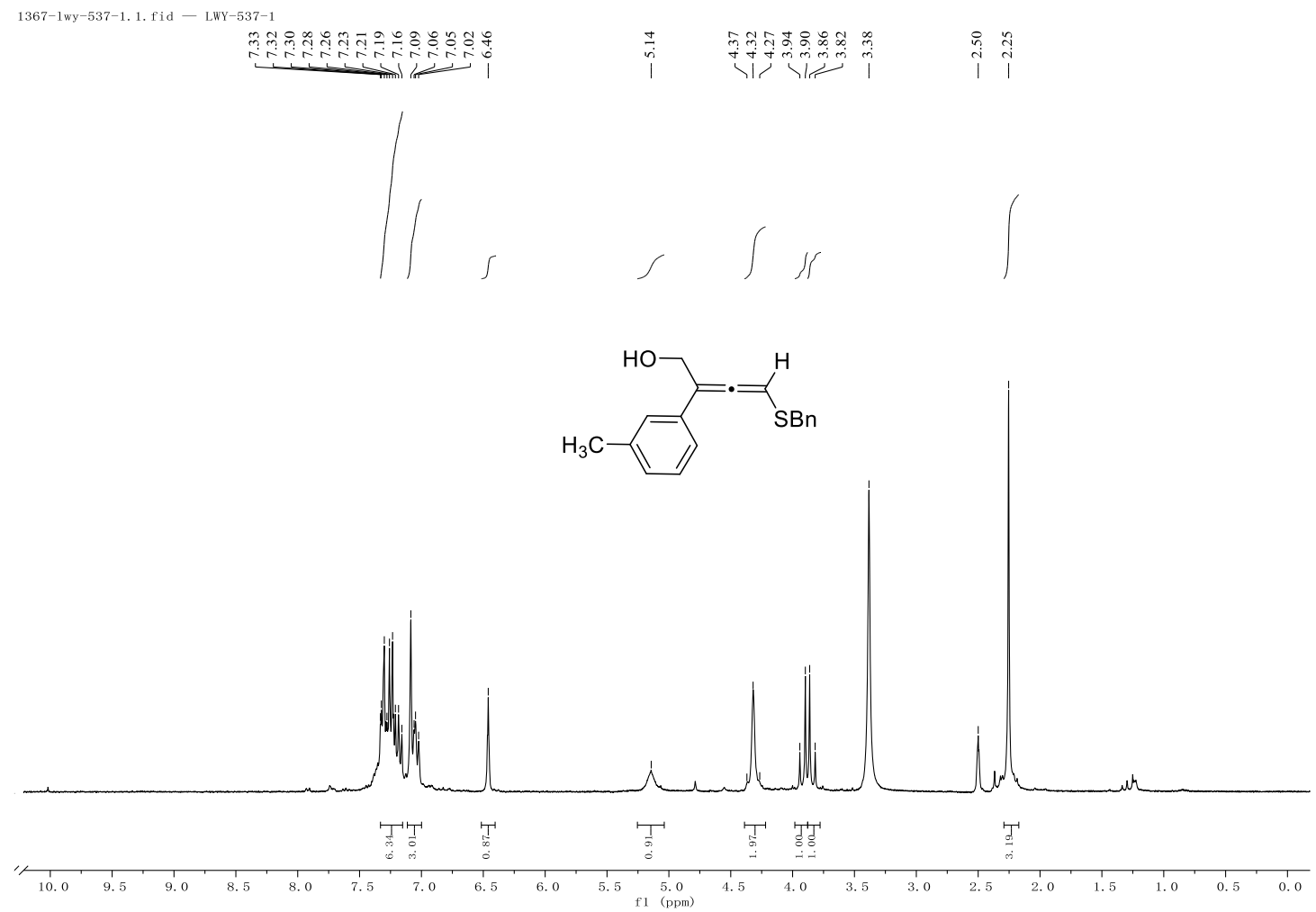

${ }^{13} \mathrm{C}\left\{{ }^{1} \mathrm{H}\right\}$ NMR (101 MHz, DMSO-d $)$ of $\mathbf{3 b}$
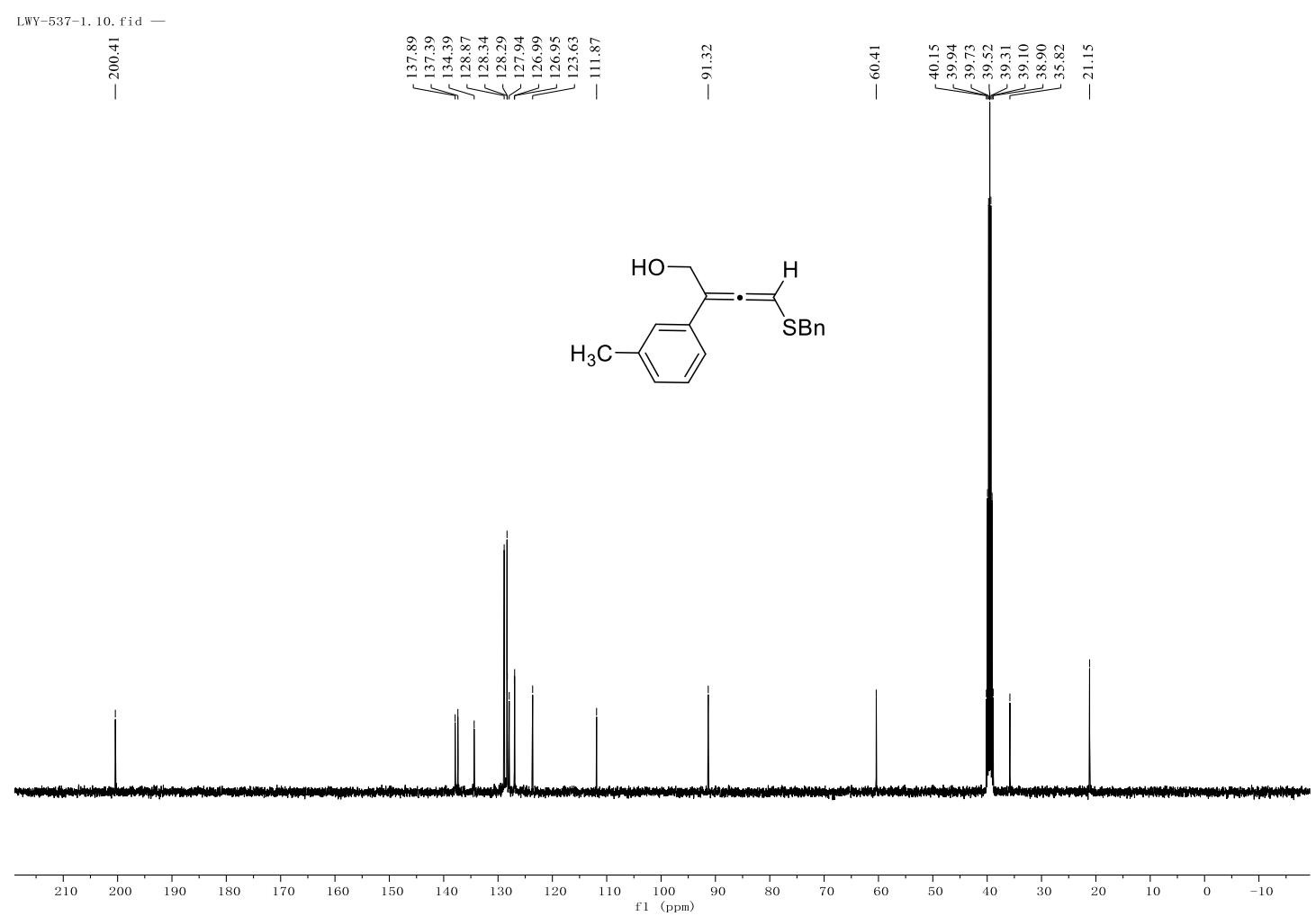
${ }^{1} \mathrm{H}$ NMR (300 MHz, DMSO- $d_{6}$ ) of $\mathbf{3 c}$

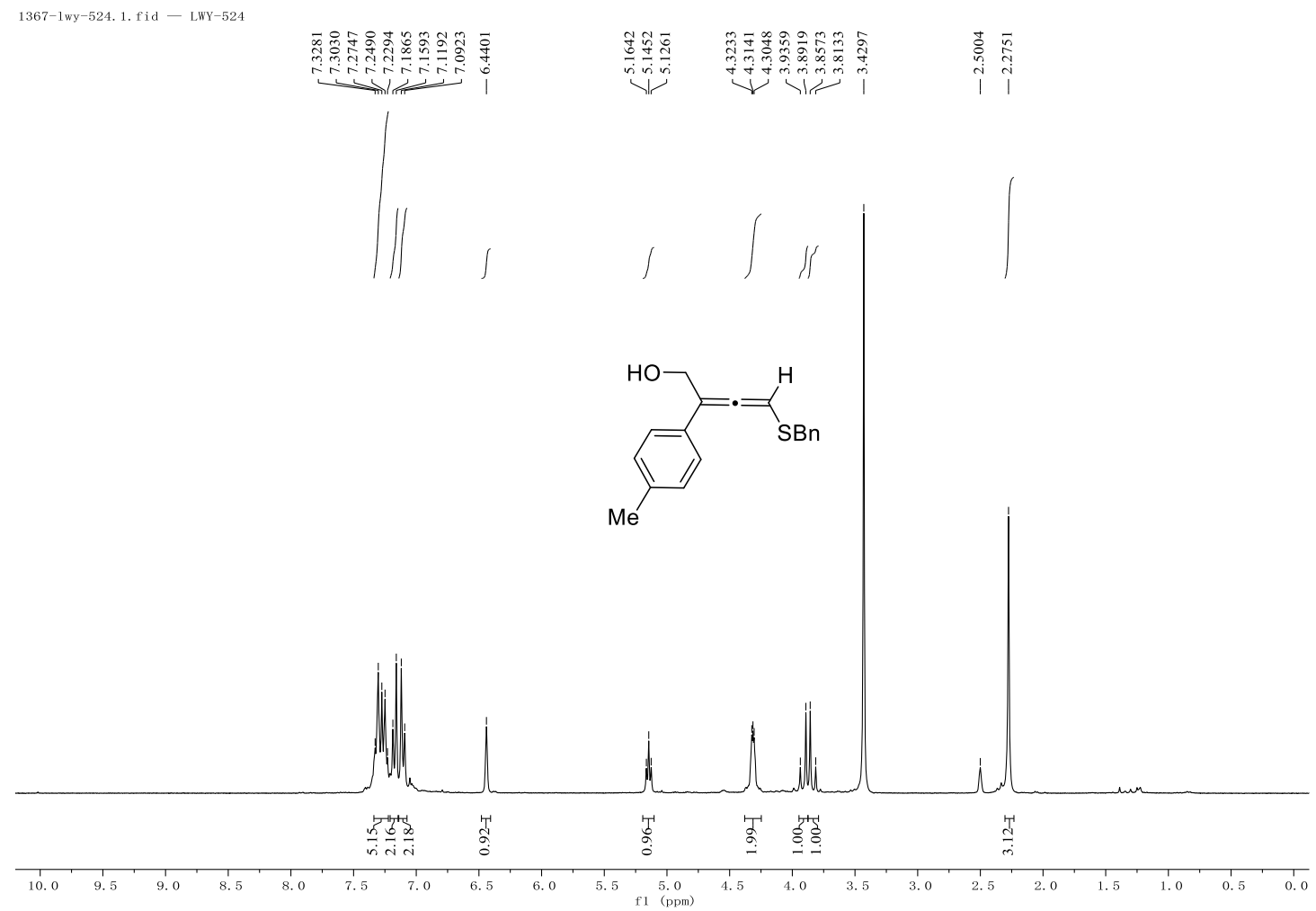

${ }^{13} \mathrm{C}\left\{{ }^{1} \mathrm{H}\right\}$ NMR (101 MHz, DMSO- $\left.d_{6}\right)$ of $\mathbf{3 c}$
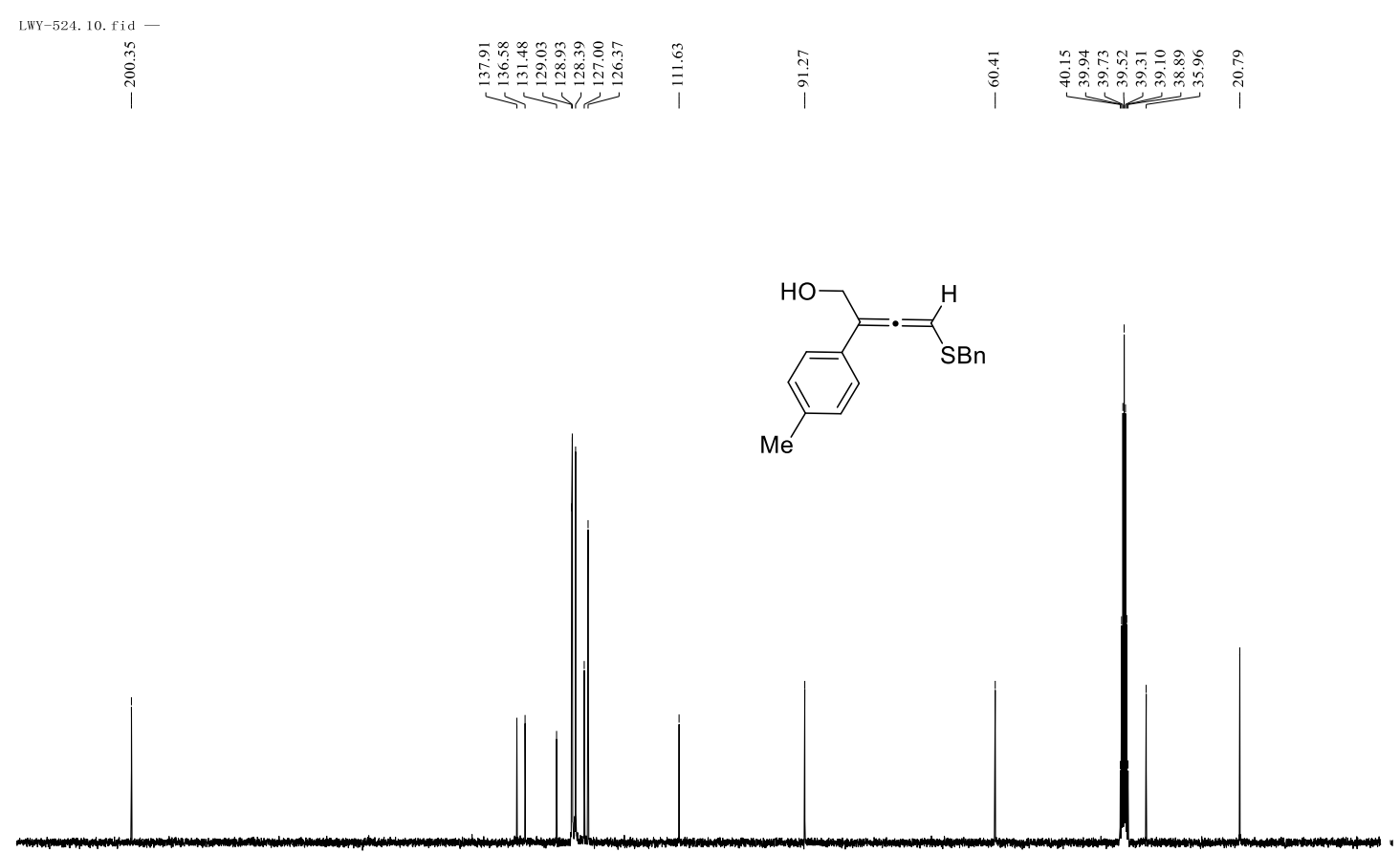
${ }^{1} \mathrm{H}$ NMR (300 MHz, DMSO- $d_{6}$ ) of $\mathbf{3 d}$

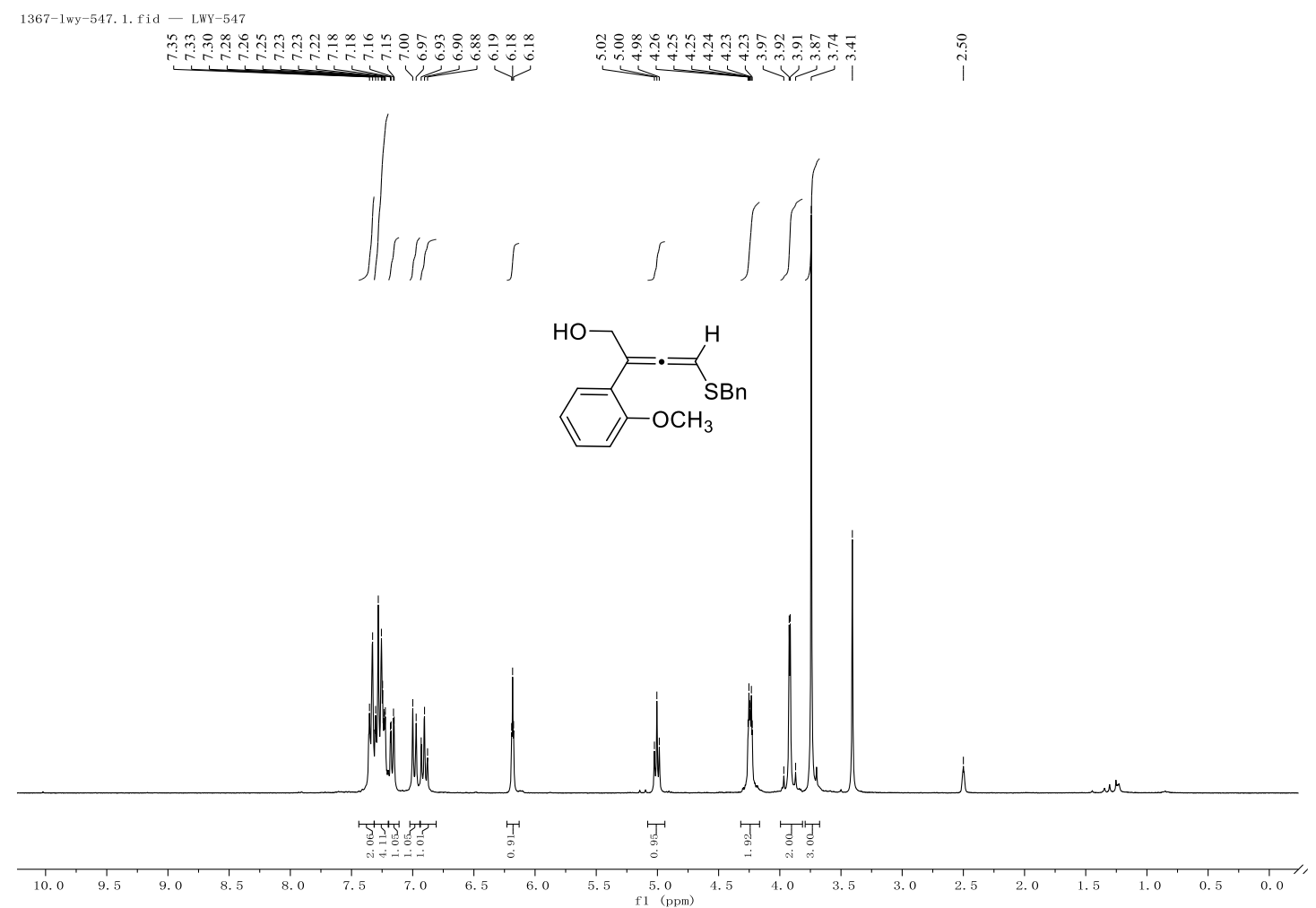

${ }^{13} \mathrm{C}\left\{{ }^{1} \mathrm{H}\right\}$ NMR $\left(101 \mathrm{MHz}\right.$, DMSO- $\left.d_{6}\right)$ of $\mathbf{3 d}$
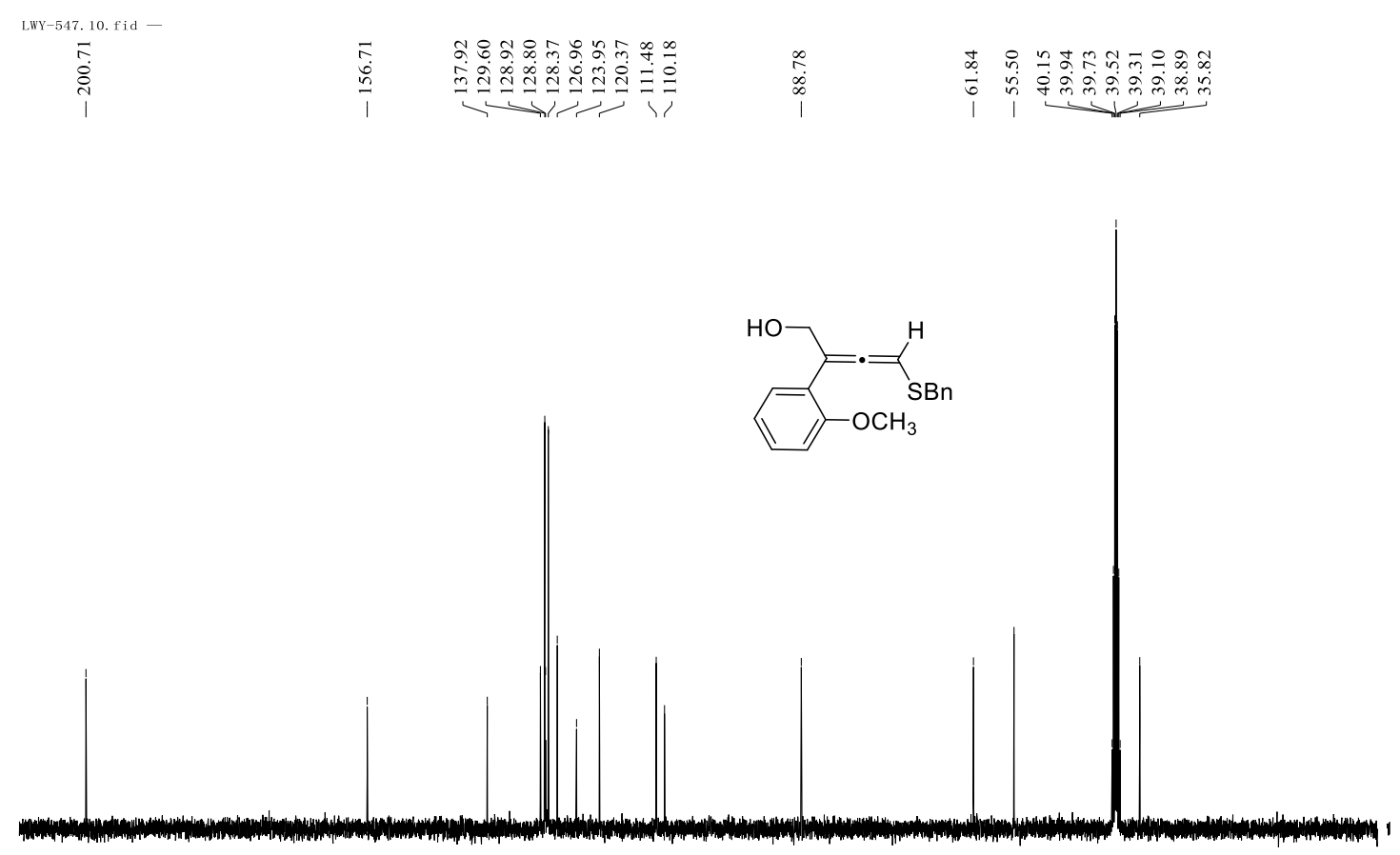

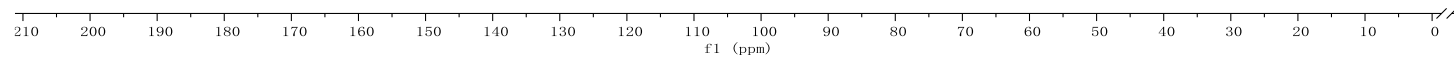


${ }^{1} \mathrm{H}$ NMR (300 MHz, DMSO- $d_{6}$ ) of $\mathbf{3 e}$

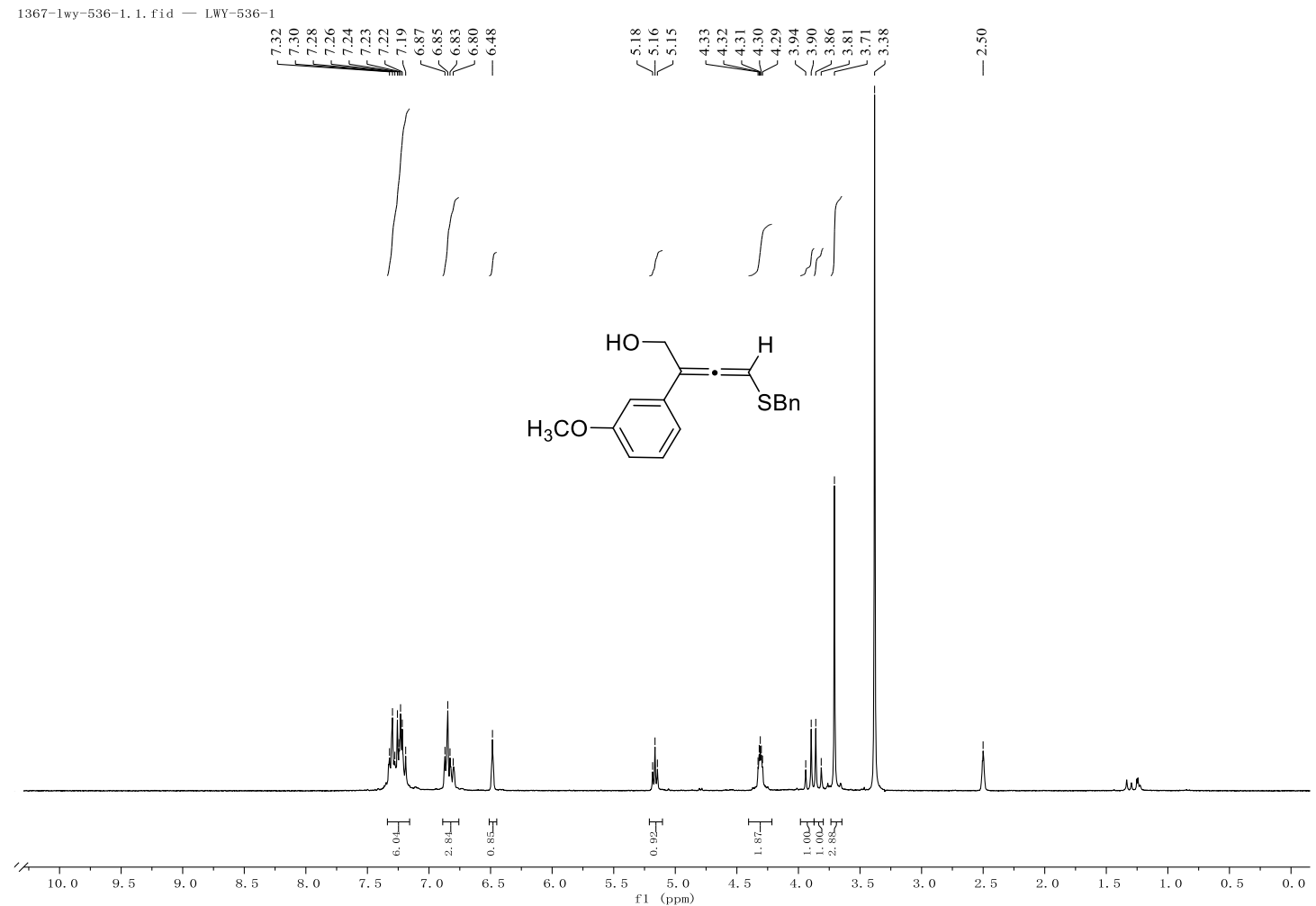

${ }^{13} \mathrm{C}\left\{{ }^{1} \mathrm{H}\right\}$ NMR (101 MHz, DMSO- $\left.d_{6}\right)$ of $\mathbf{3 e}$

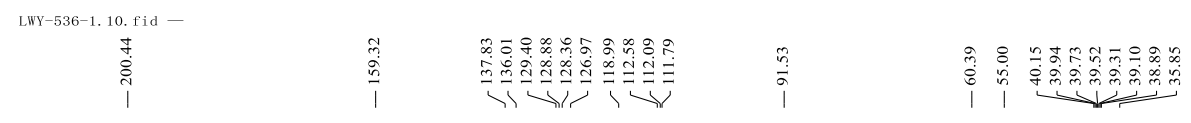
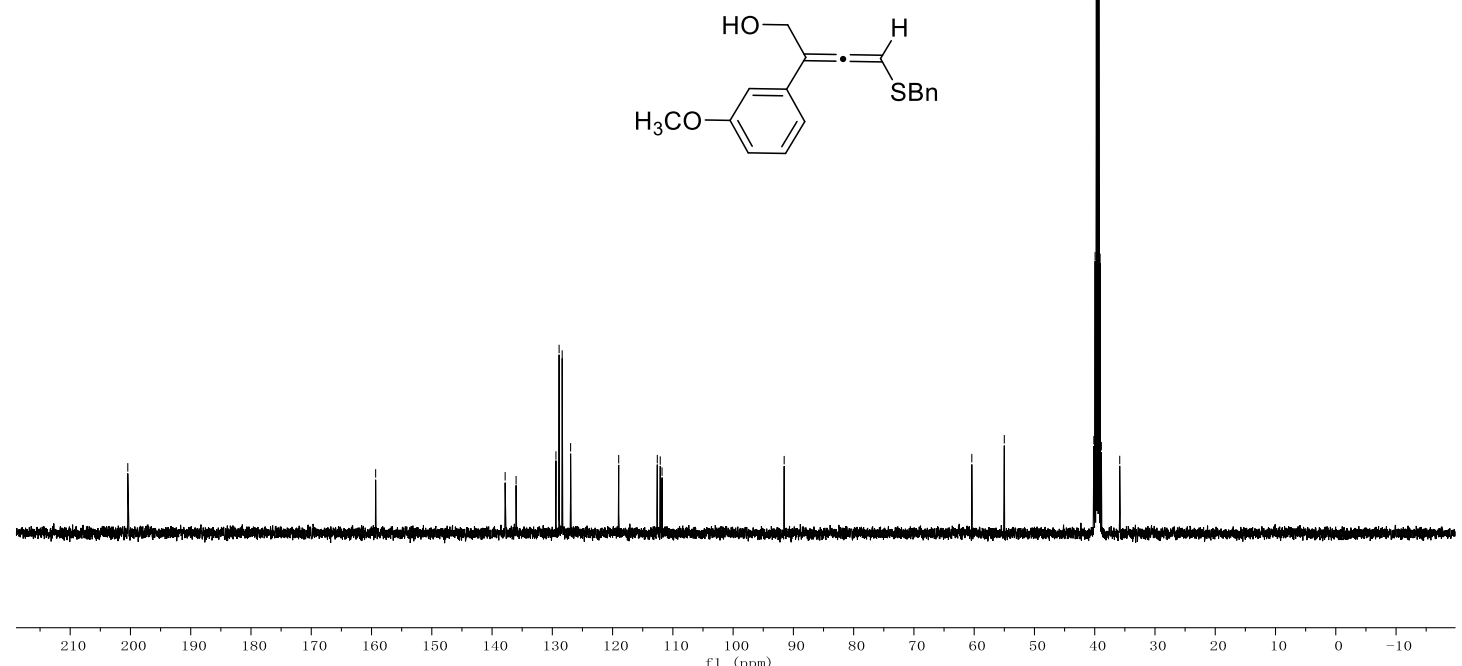
${ }^{1} \mathrm{H}$ NMR (300 MHz, DMSO- $\left.d_{6}\right)$ of $\mathbf{3 f}$

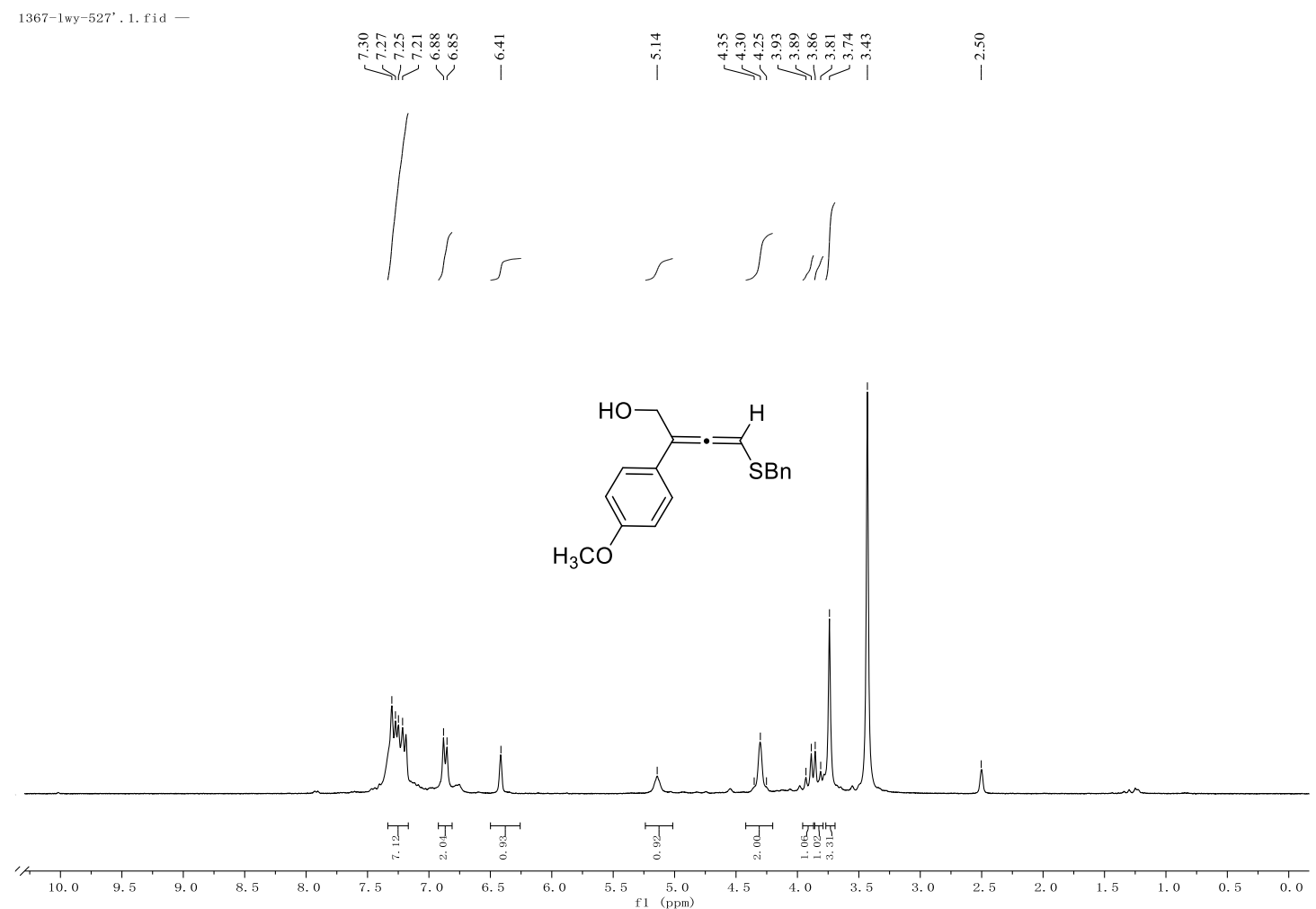

${ }^{13} \mathrm{C}\left\{{ }^{1} \mathrm{H}\right\}$ NMR (151 MHz, DMSO- $\left.d_{6}\right)$ of $\mathbf{3 f}$

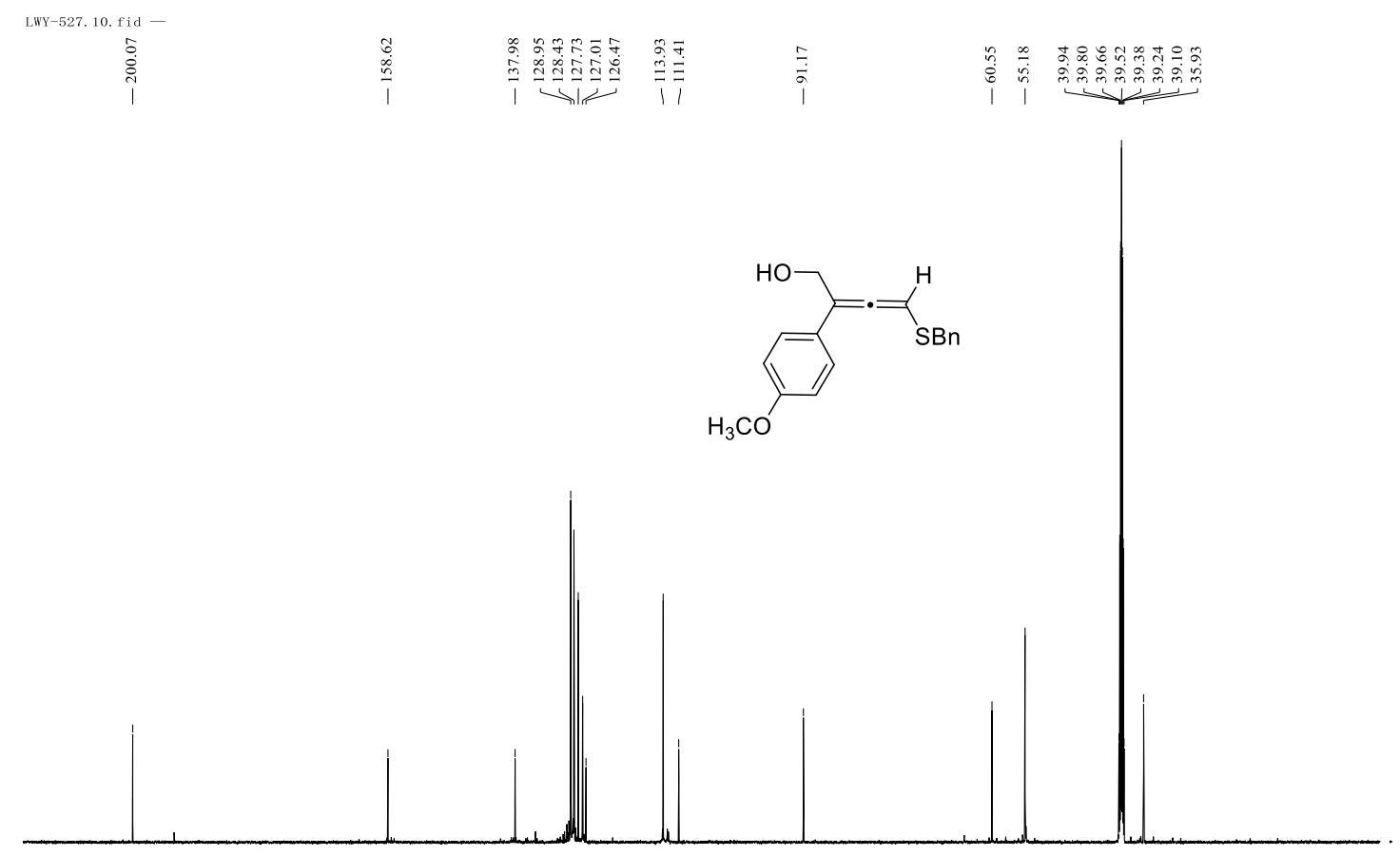

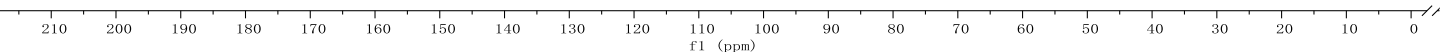


${ }^{1} \mathrm{H}$ NMR (300 MHz, DMSO- $d_{6}$ ) of $\mathbf{3 g}$

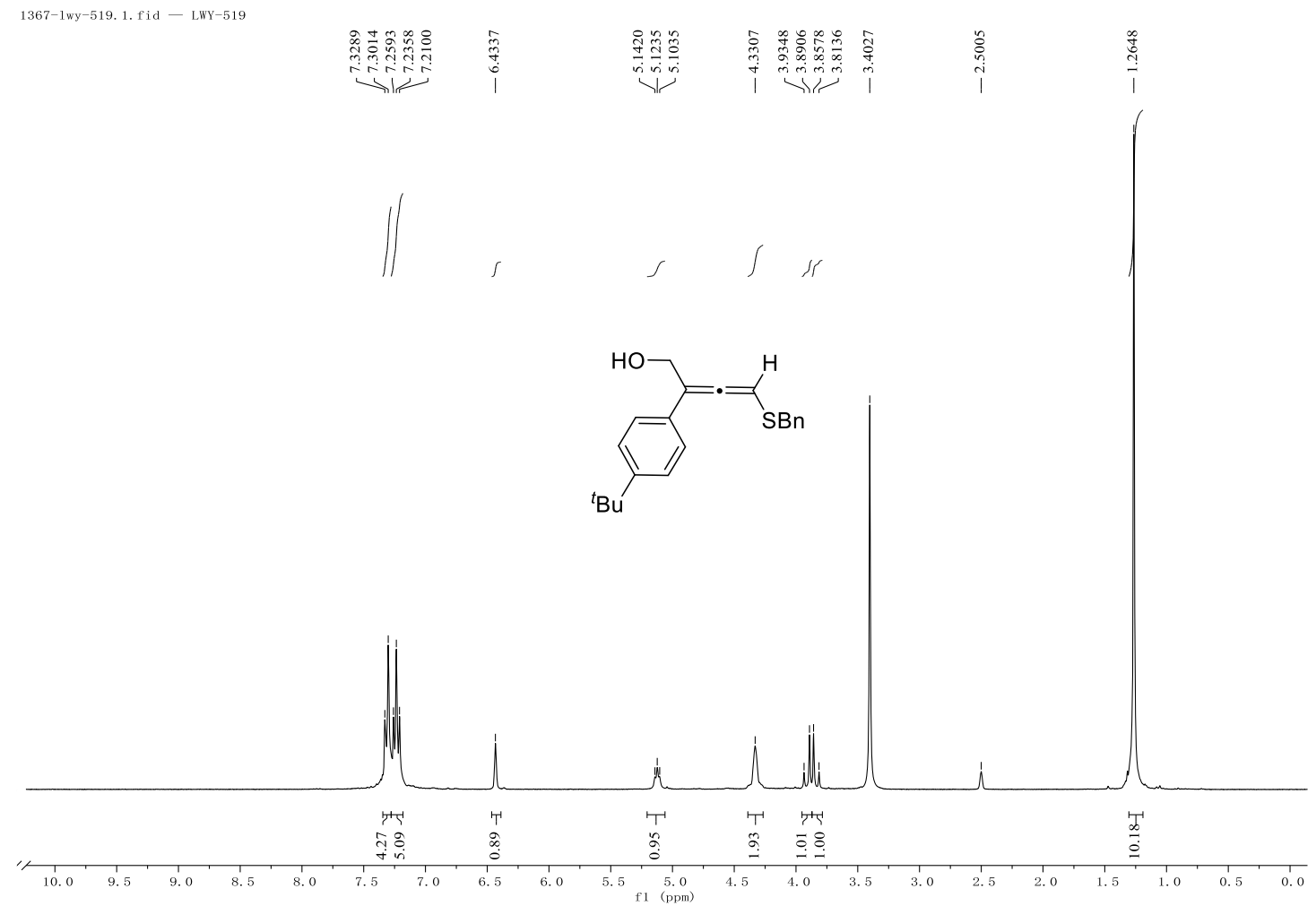

${ }^{13} \mathrm{C}\left\{{ }^{1} \mathrm{H}\right\}$ NMR (151 MHz, DMSO- $\left.d_{6}\right)$ of $\mathbf{3 g}$

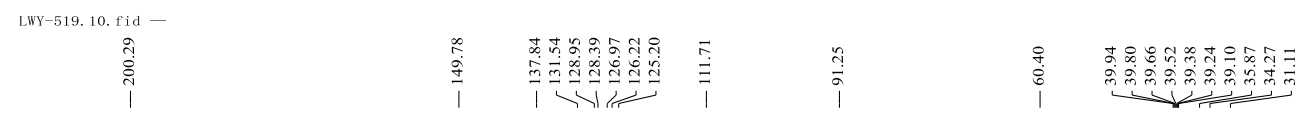
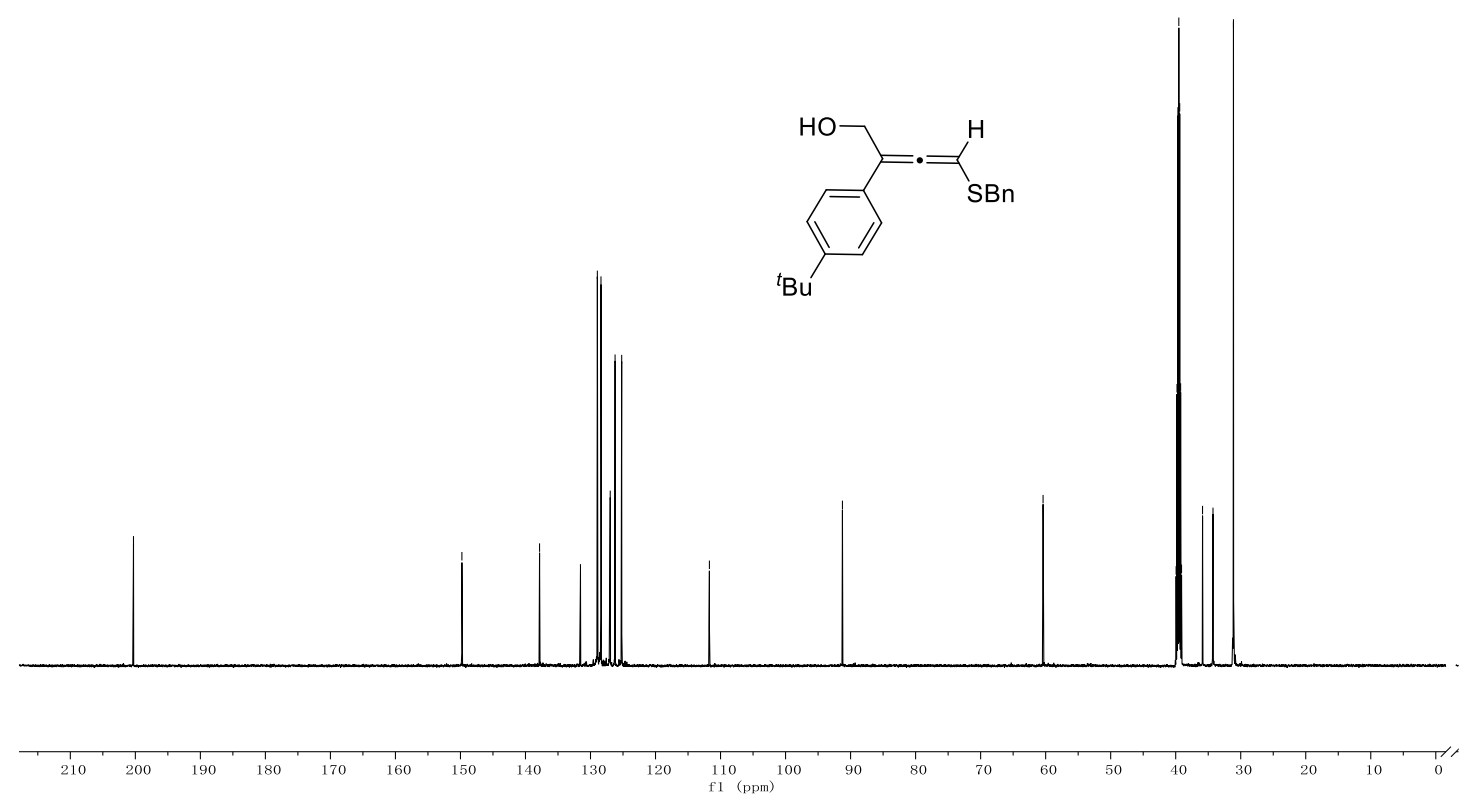
${ }^{1} \mathrm{H}$ NMR (300 MHz, DMSO- $d_{6}$ ) of $\mathbf{3 h}$

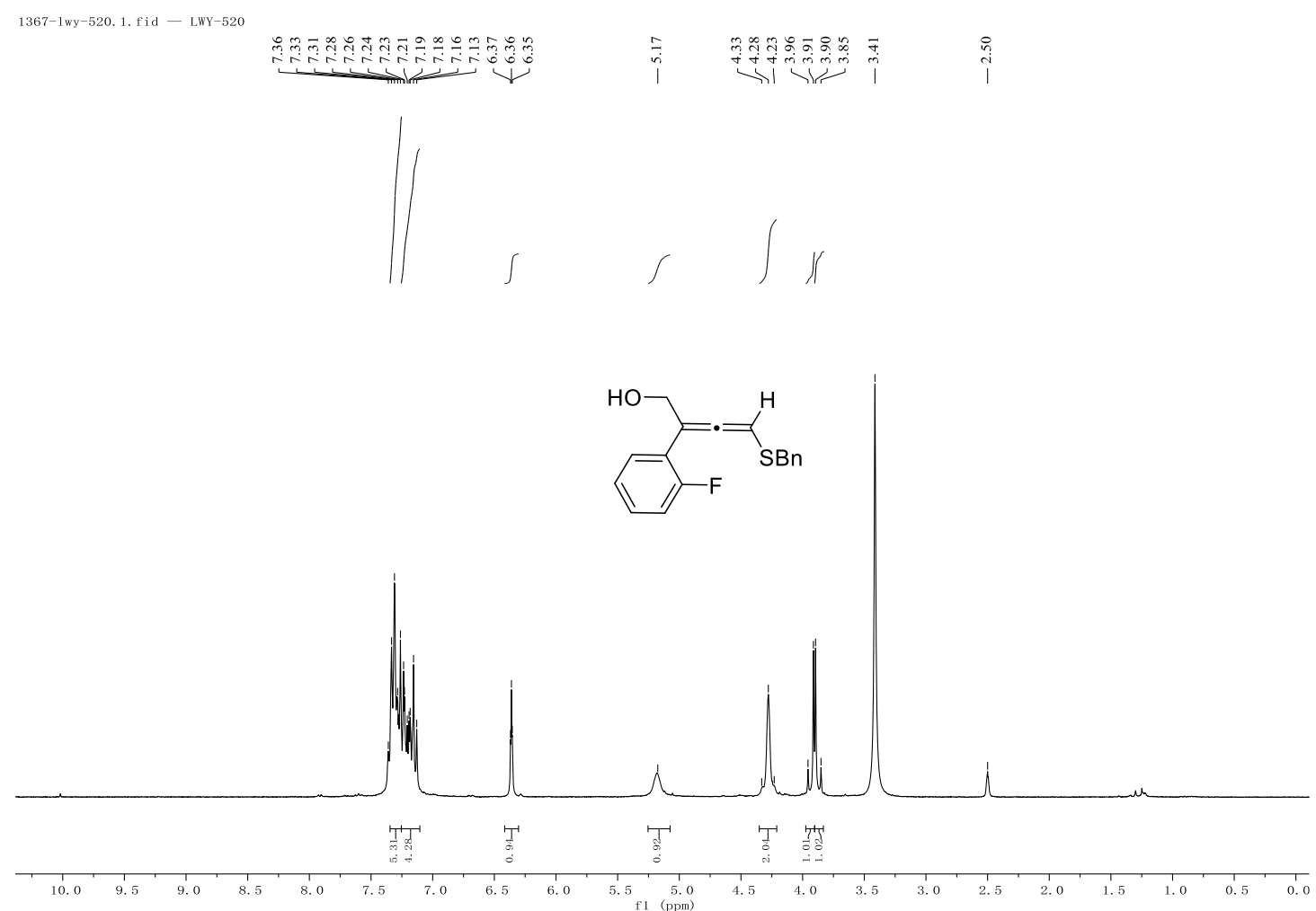

${ }^{13} \mathrm{C}\left\{{ }^{1} \mathrm{H}\right\}$ NMR $\left(151 \mathrm{MHz}\right.$, DMSO- $\left.d_{6}\right)$ of $\mathbf{3 h}$

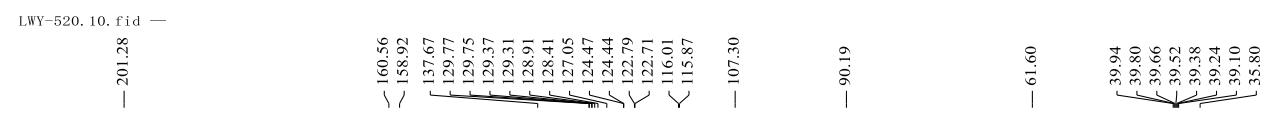
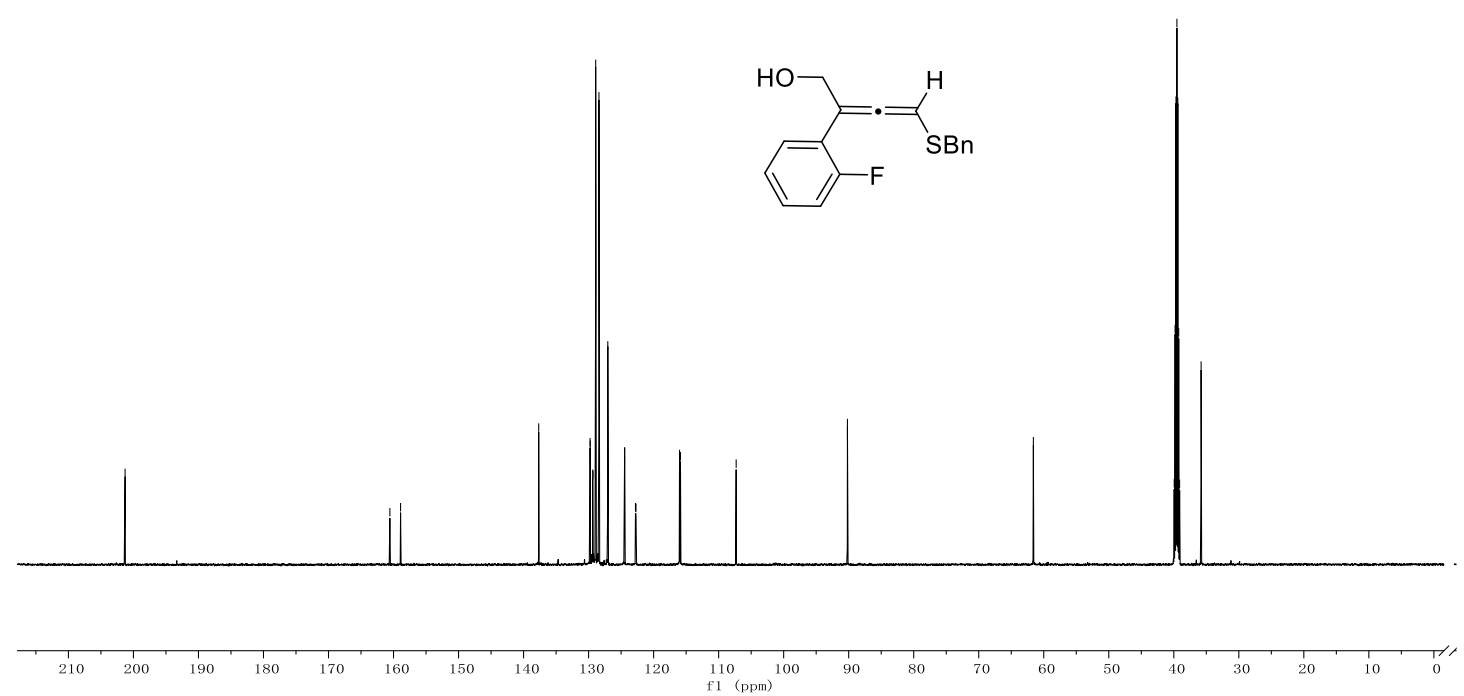
${ }^{1} \mathrm{H}$ NMR (300 MHz, DMSO- $\left.d_{6}\right)$ of $\mathbf{3 i}$

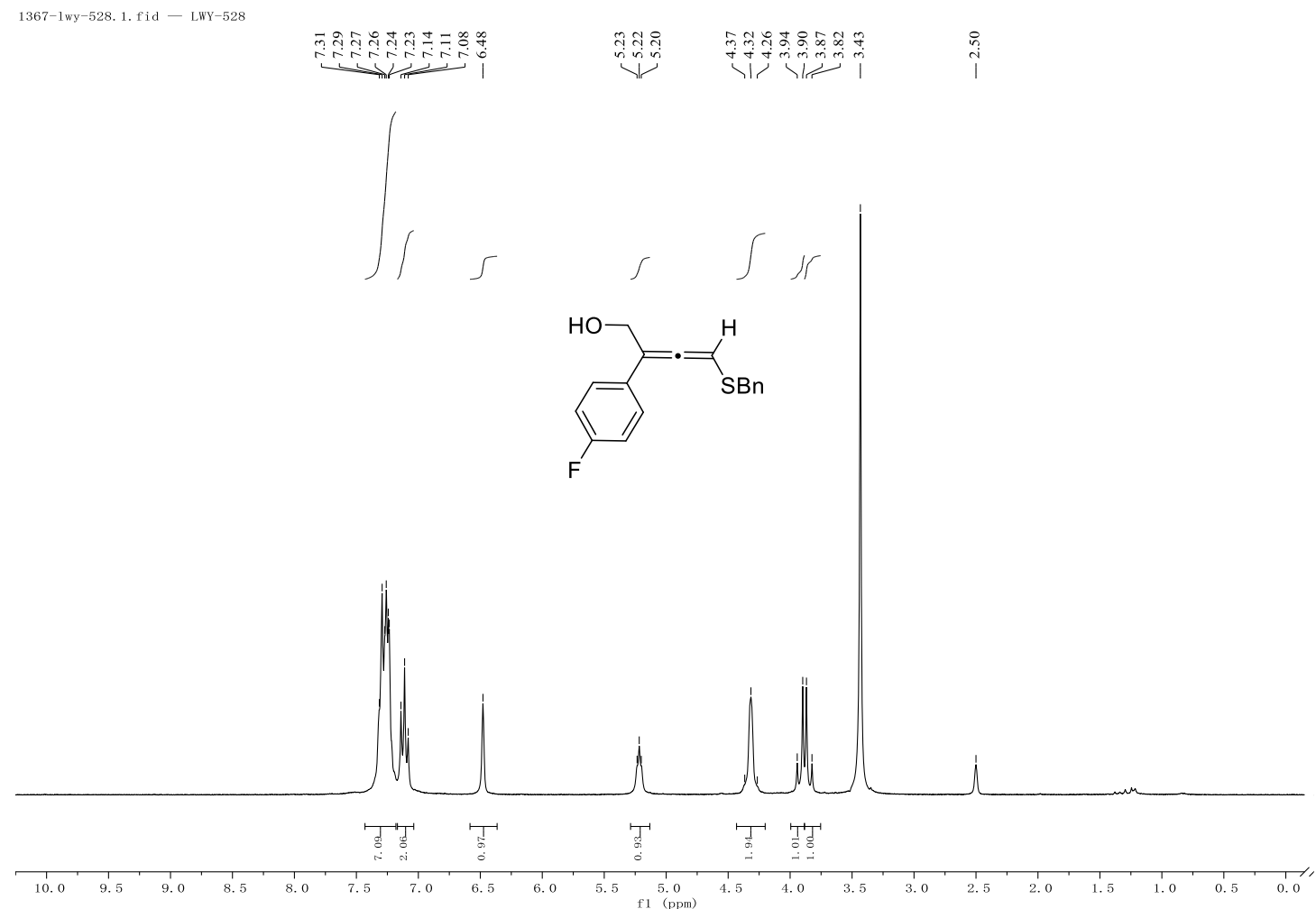

${ }^{13} \mathrm{C}\left\{{ }^{1} \mathrm{H}\right\}$ NMR (151 MHz, DMSO- $\left.d_{6}\right)$ of $\mathbf{3 i}$

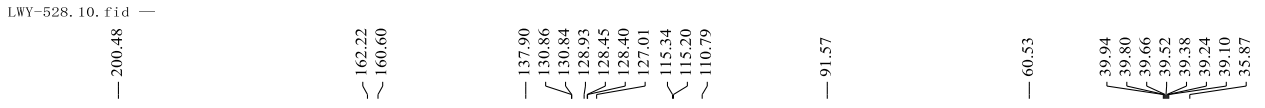
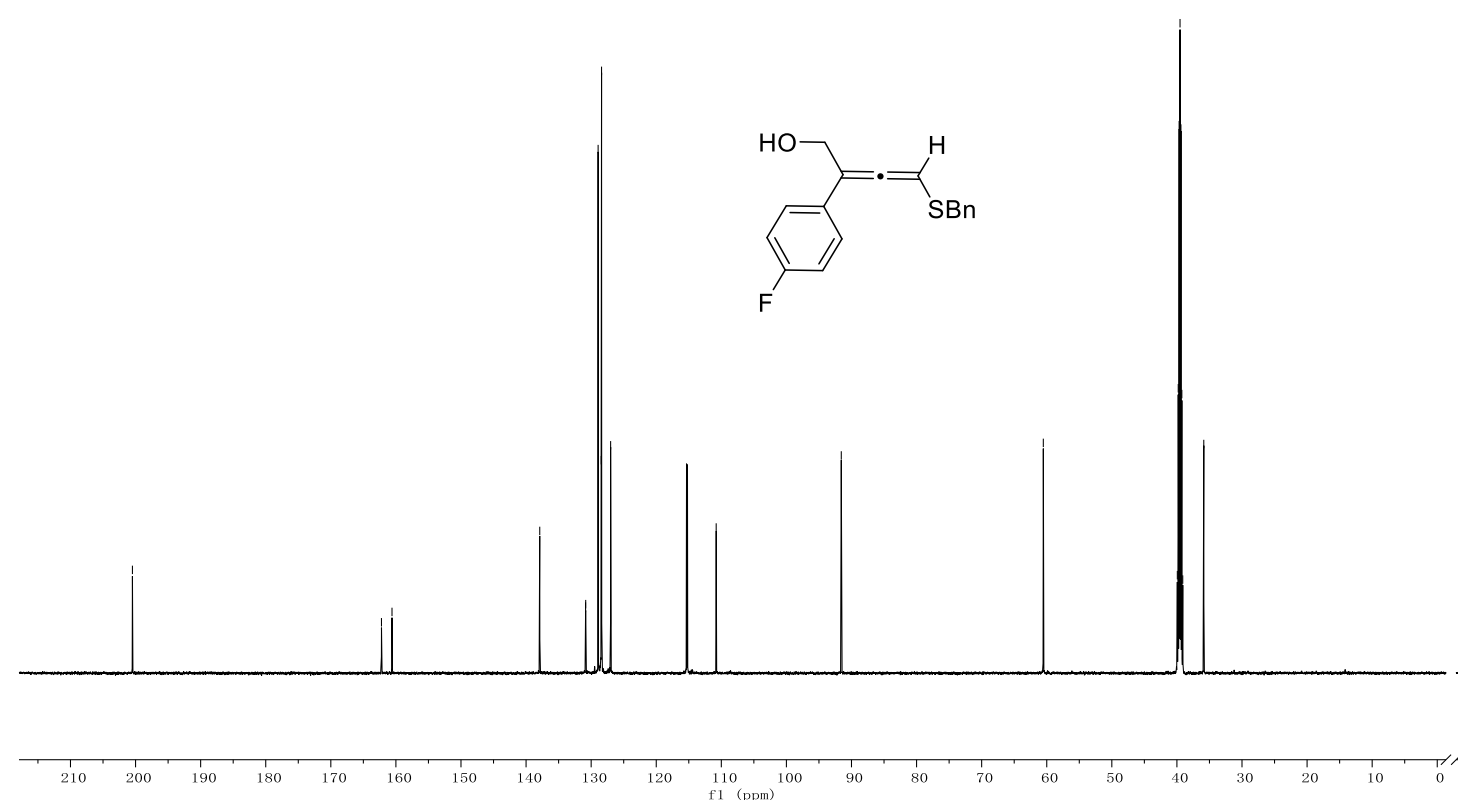
${ }^{1} \mathrm{H}$ NMR (300 MHz, DMSO- $d_{6}$ ) of $\mathbf{3 j}$

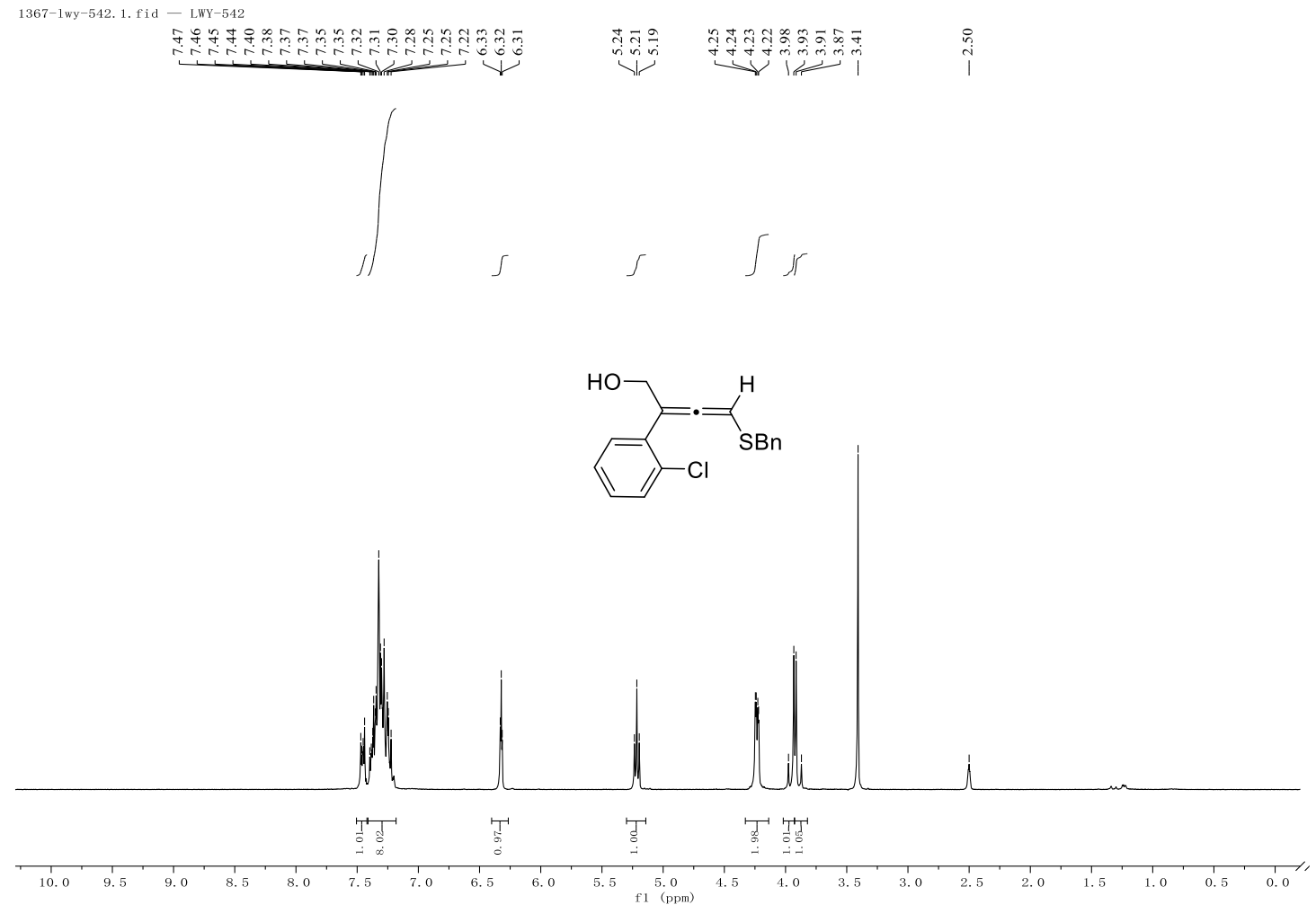

${ }^{13} \mathrm{C}\left\{{ }^{1} \mathrm{H}\right\}$ NMR (101 MHz, DMSO- $\left.d_{6}\right)$ of $\mathbf{3 j}$

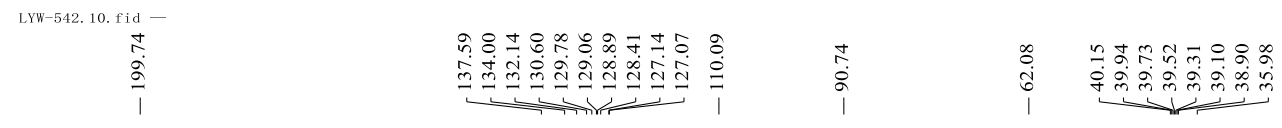

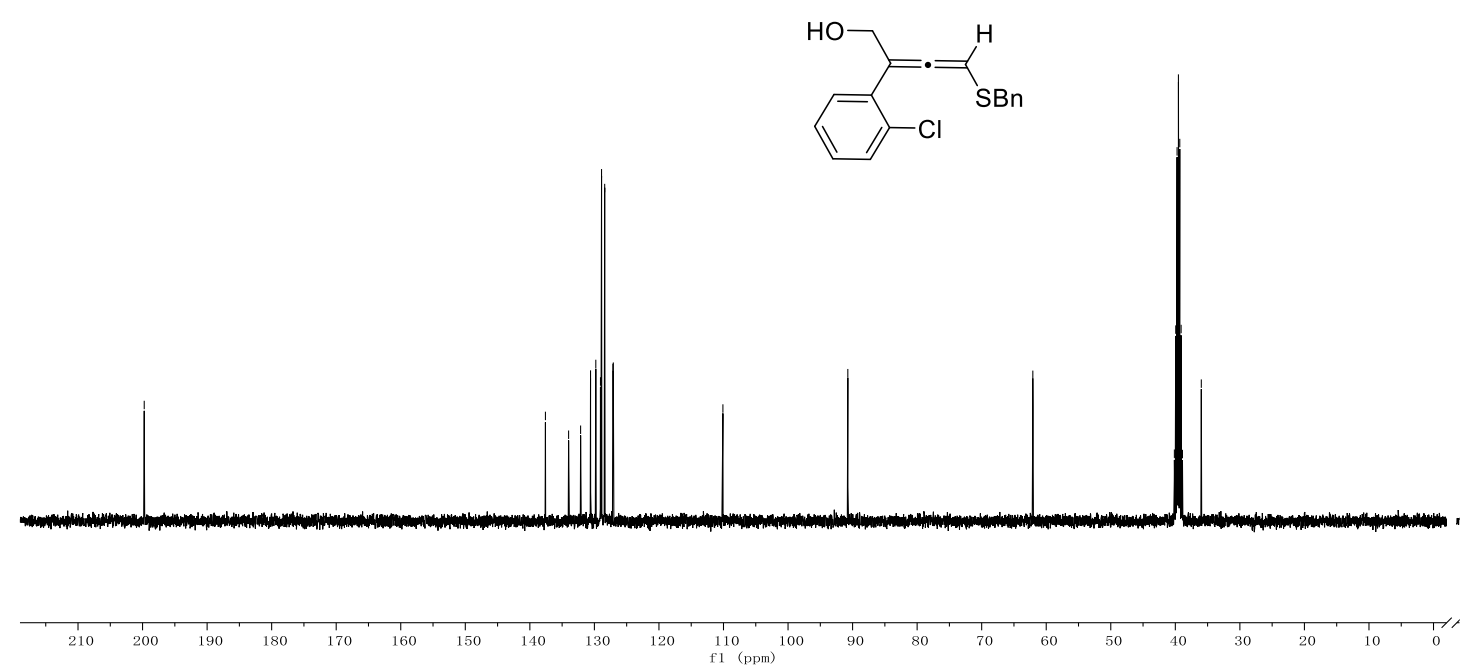


${ }^{1} \mathrm{H}$ NMR (400 MHz, DMSO- $d_{6}$ ) of $\mathbf{3 k}$

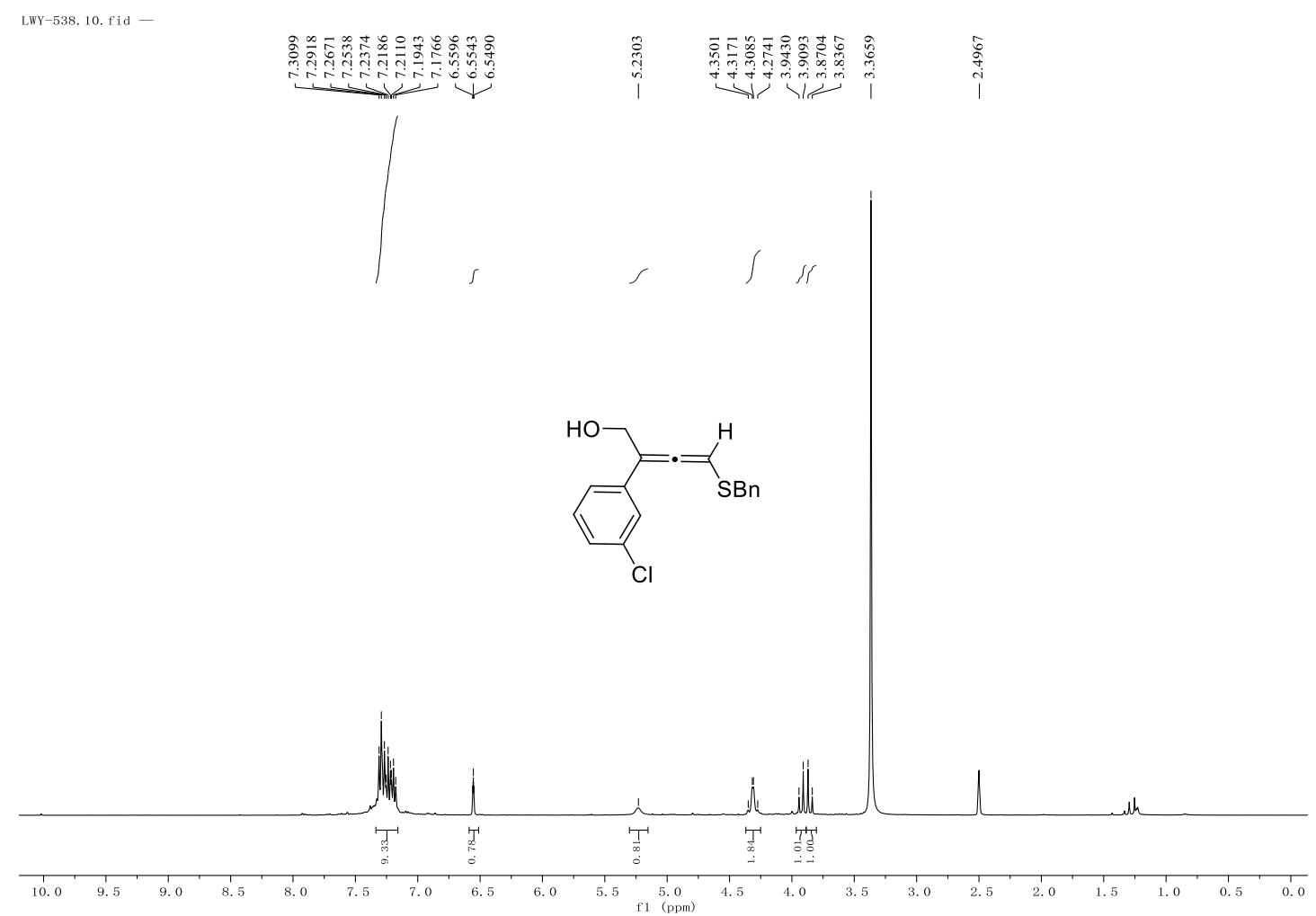

${ }^{13} \mathrm{C}\left\{{ }^{1} \mathrm{H}\right\}$ NMR (101 MHz, DMSO- $\left.d_{6}\right)$ of $\mathbf{3 k}$
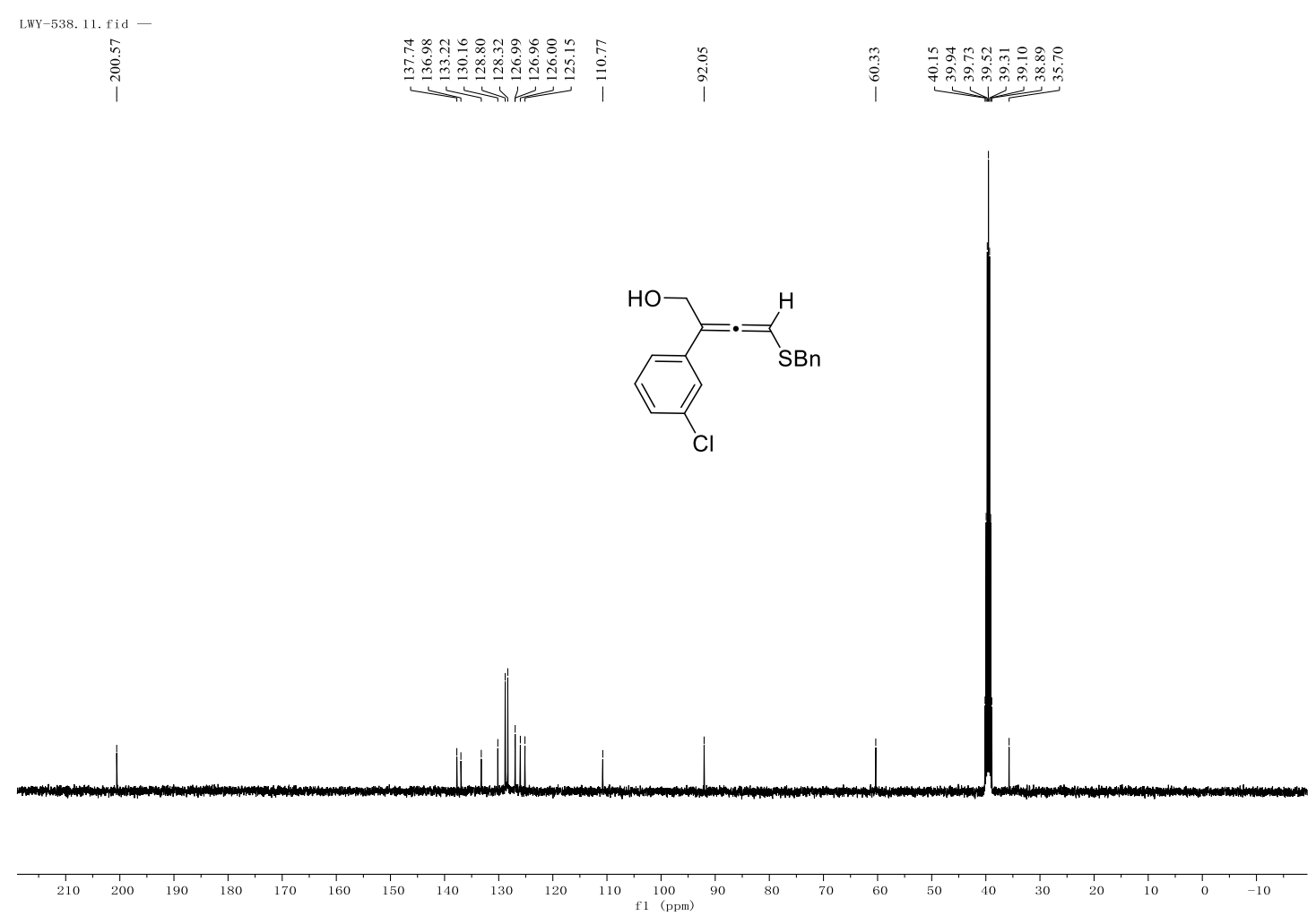
${ }^{1} \mathrm{H}$ NMR (300 MHz, DMSO- $d_{6}$ ) of $\mathbf{3 I}$

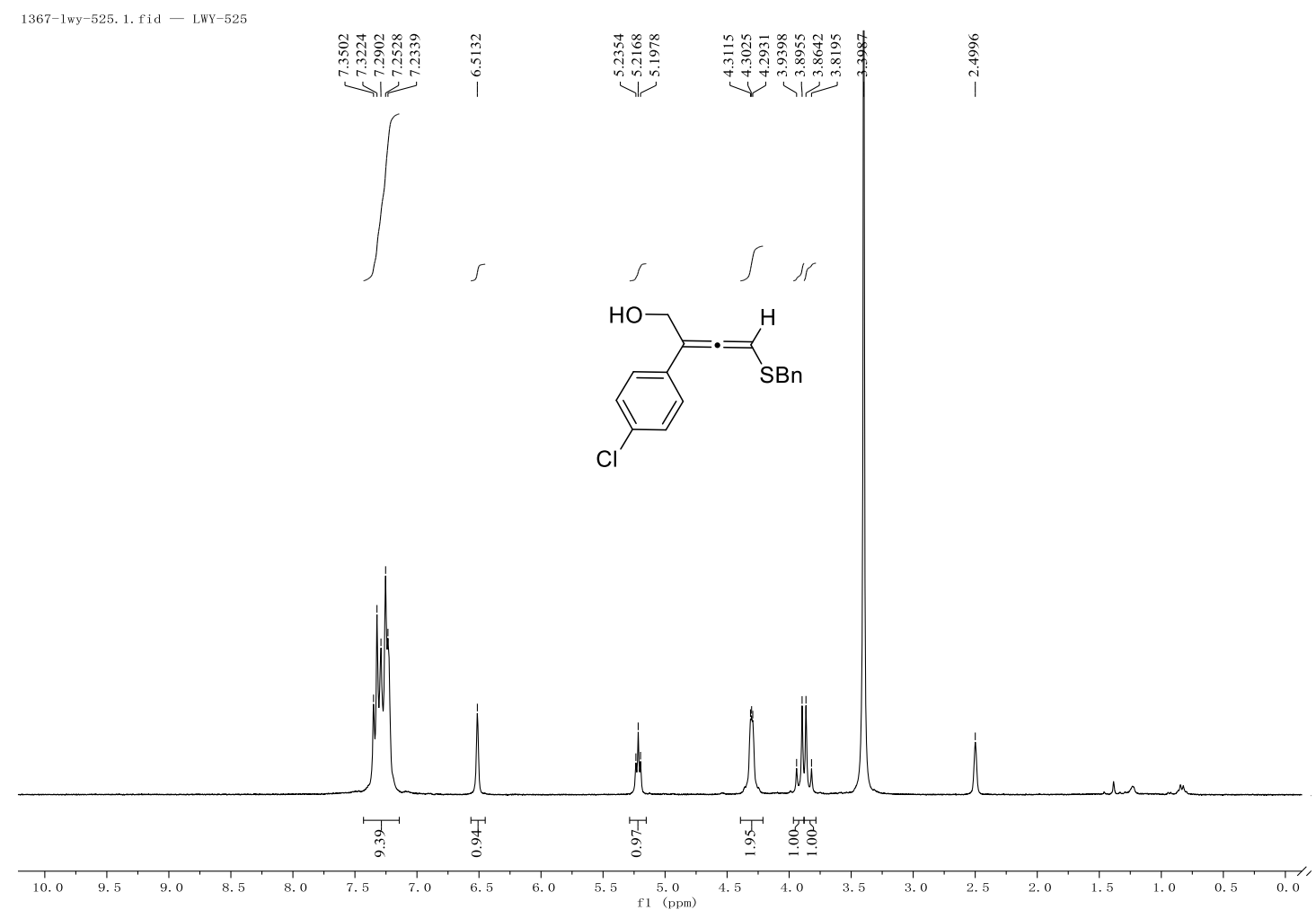

${ }^{13} \mathrm{C}\left\{{ }^{1} \mathrm{H}\right\}$ NMR (101 MHz, DMSO- $d_{6}$ ) of 31

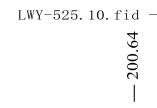

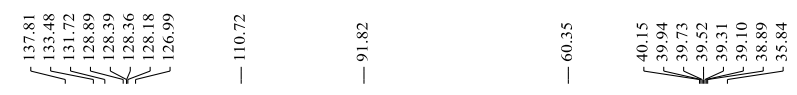

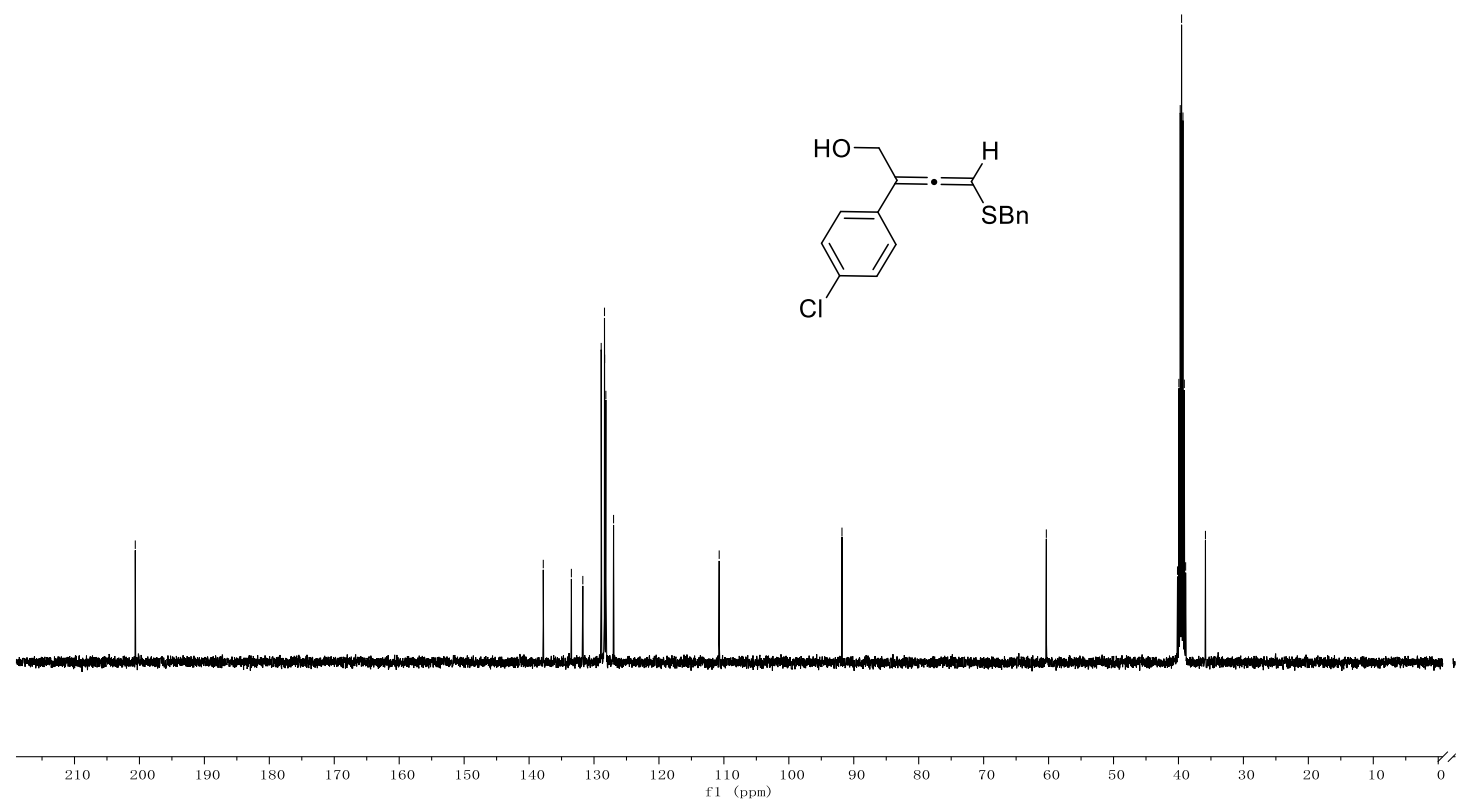


${ }^{1} \mathrm{H}$ NMR (300 MHz, DMSO- $d_{6}$ ) of 3m

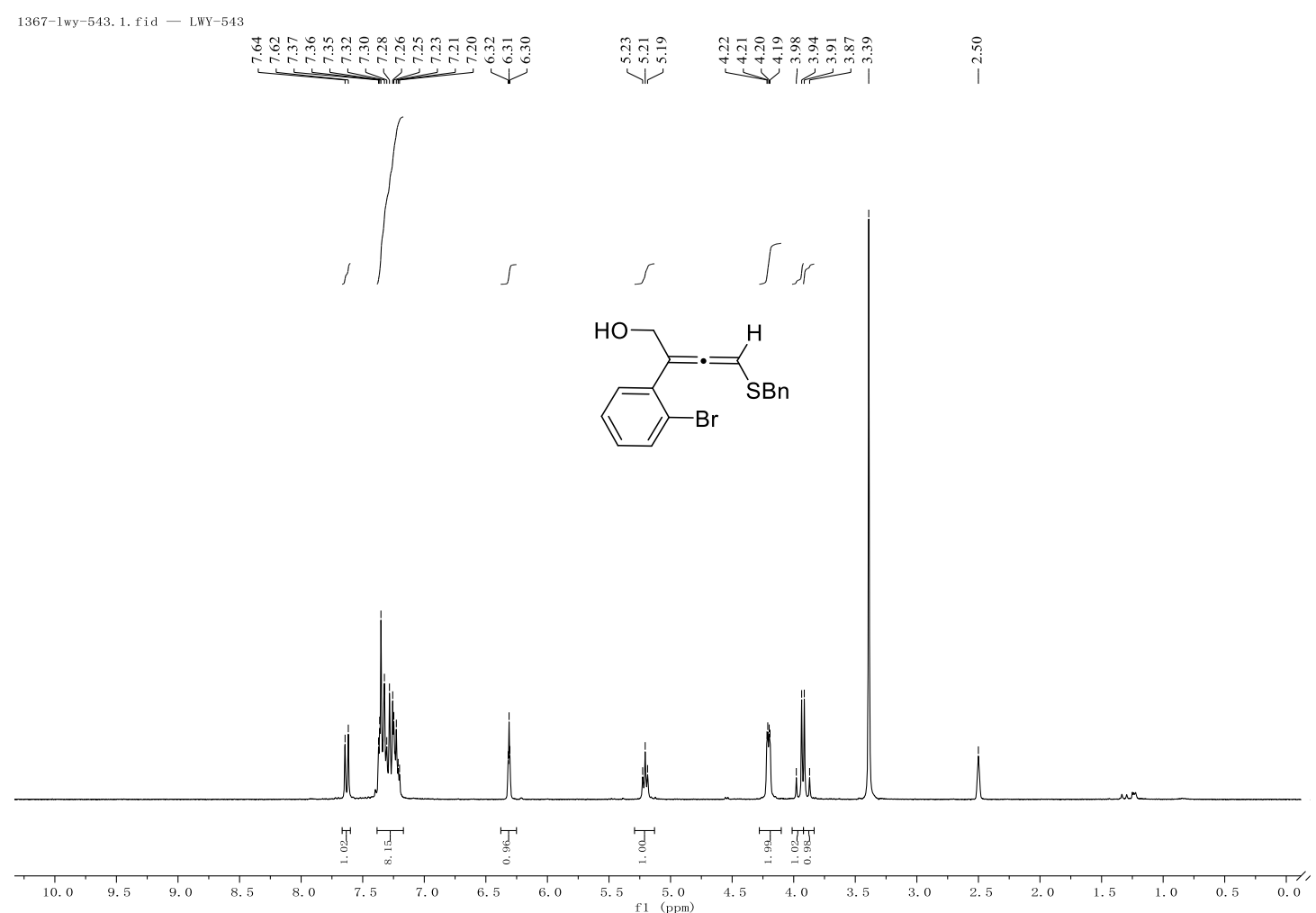

${ }^{13} \mathrm{C}\left\{{ }^{1} \mathrm{H}\right\}$ NMR (101 MHz, DMSO- $\left.d_{6}\right)$ of $\mathbf{3 m}$

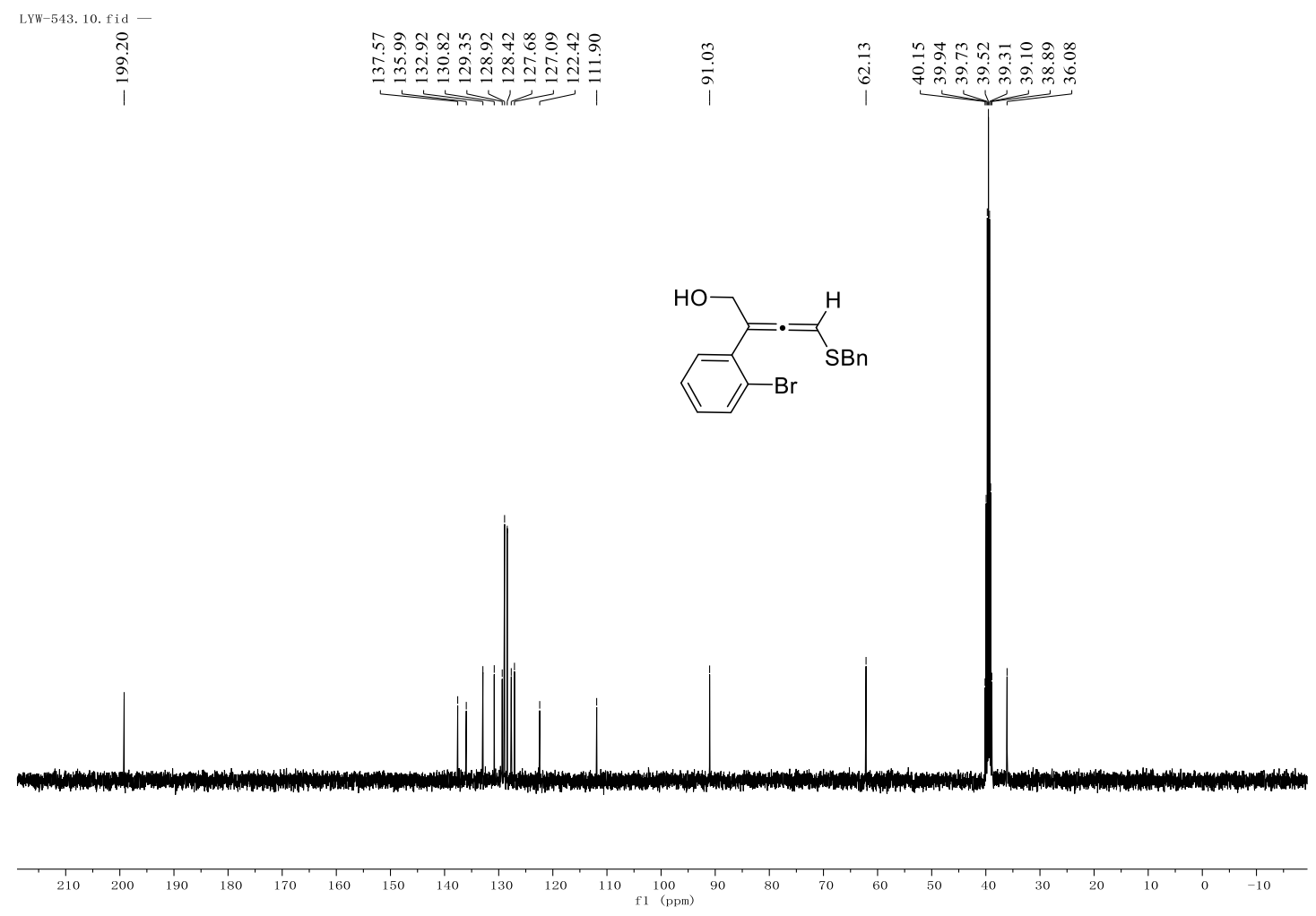


${ }^{1} \mathrm{H}$ NMR (300 MHz, DMSO- $\left.d_{6}\right)$ of $\mathbf{3 n}$

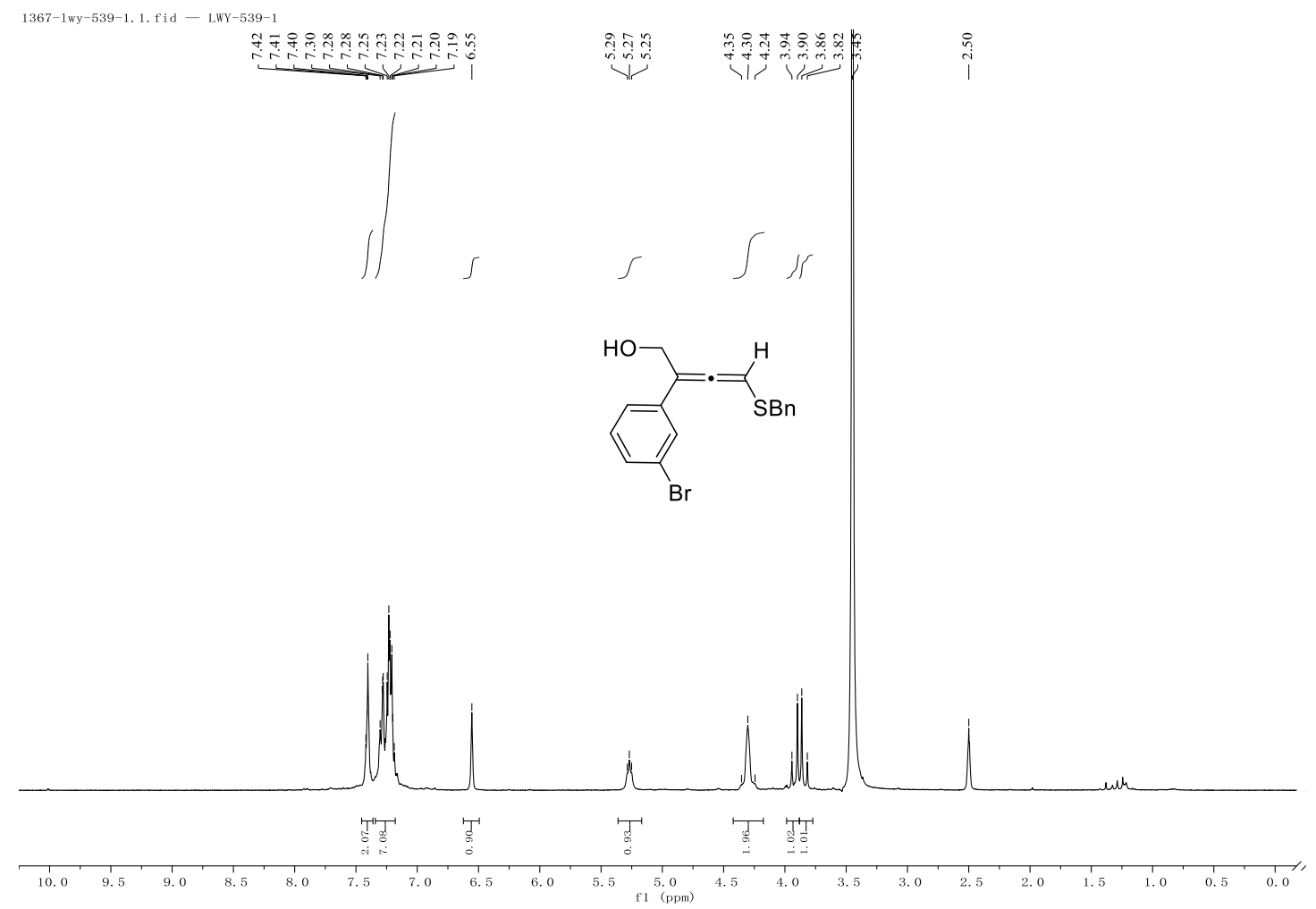

${ }^{13} \mathrm{C}\left\{{ }^{1} \mathrm{H}\right\}$ NMR (101 MHz, DMSO- $\left.d_{6}\right)$ of $\mathbf{3 n}$

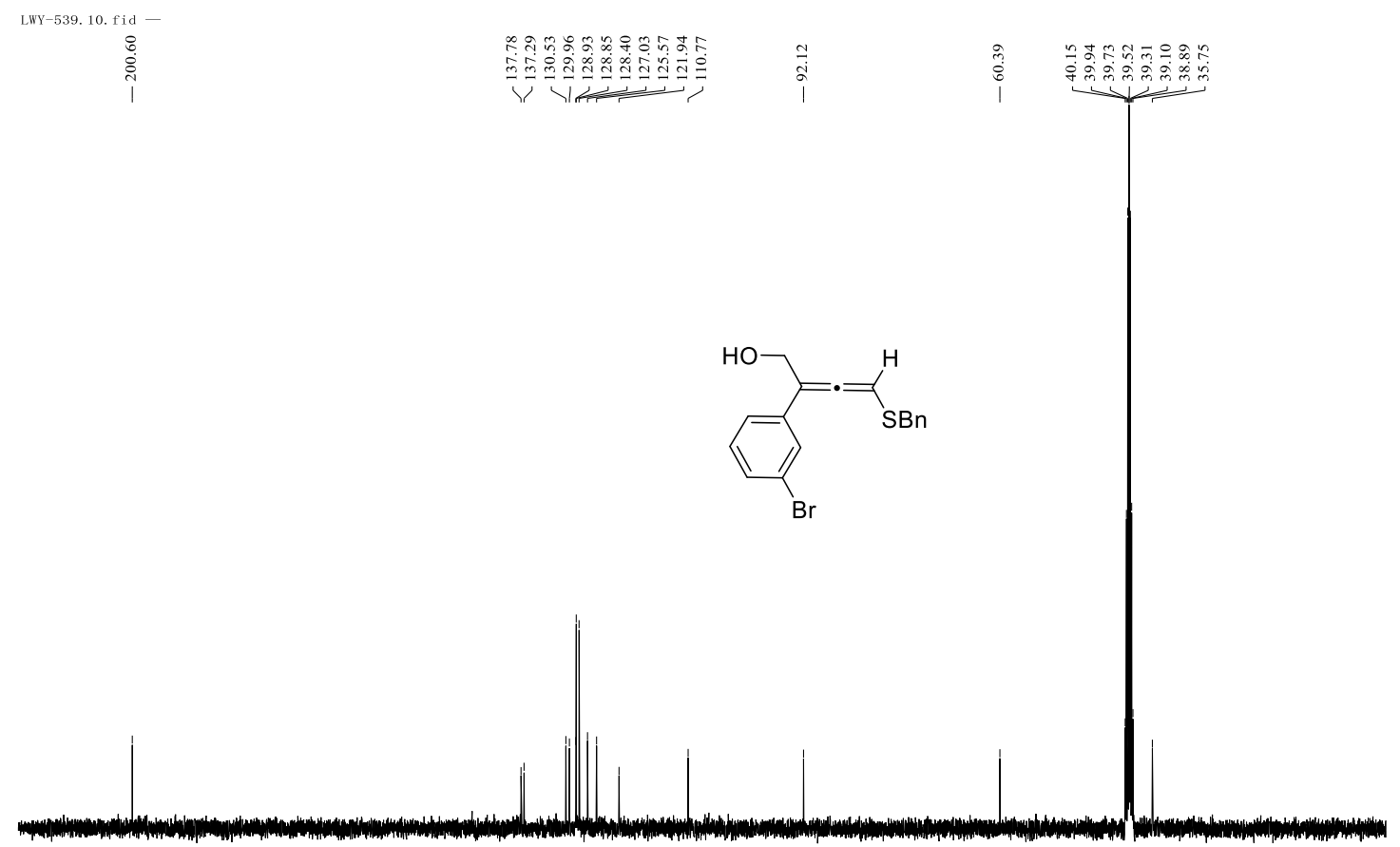

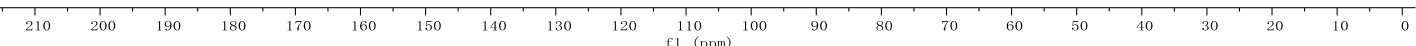


${ }^{1} \mathrm{H}$ NMR (300 MHz, DMSO- $d_{6}$ ) of $\mathbf{3 o}$

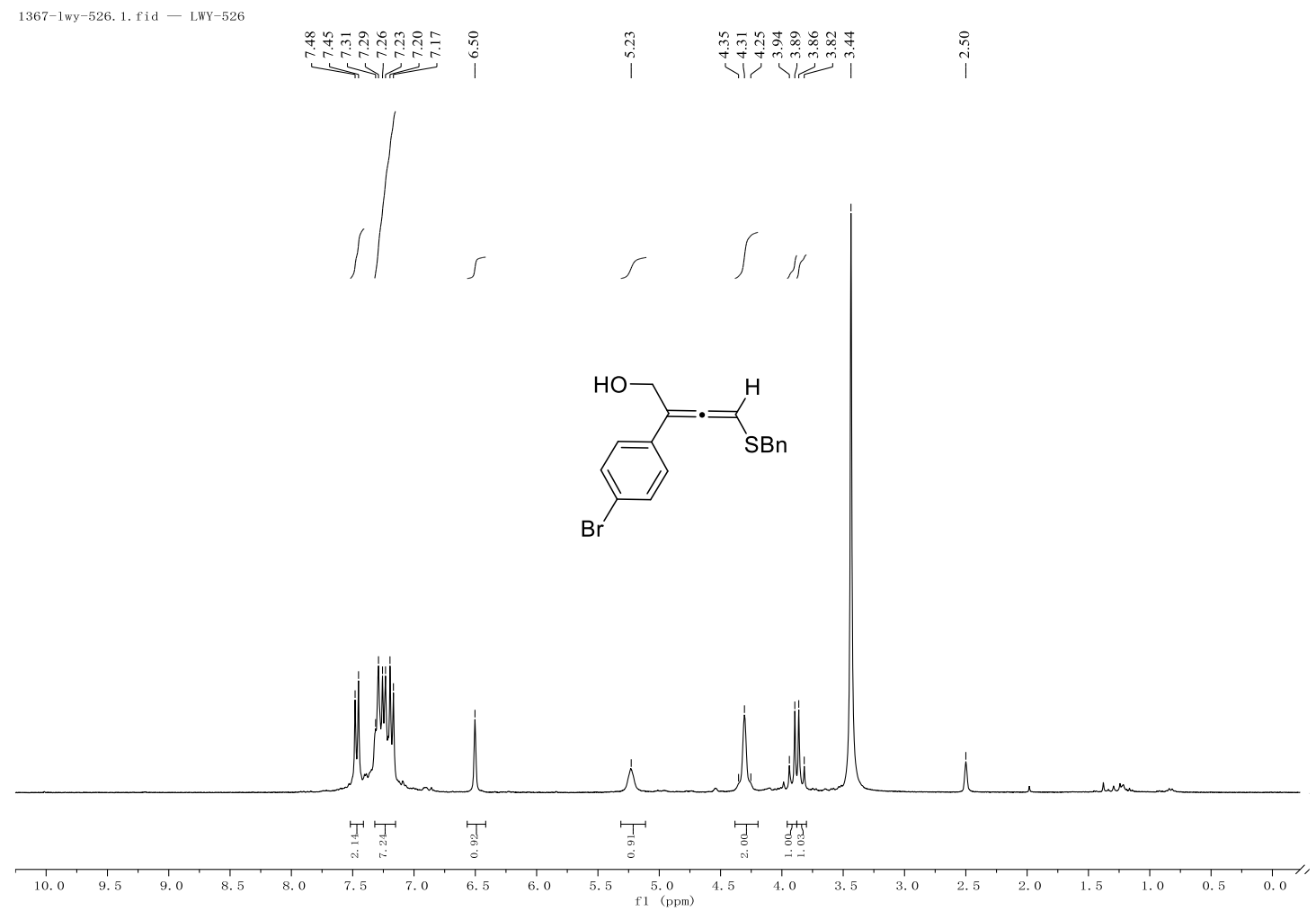

${ }^{13} \mathrm{C}\left\{{ }^{1} \mathrm{H}\right\}$ NMR (101 MHz, DMSO- $\left.d_{6}\right)$ of $3 \mathbf{0}$

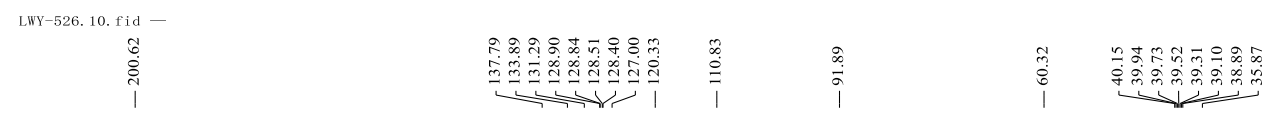

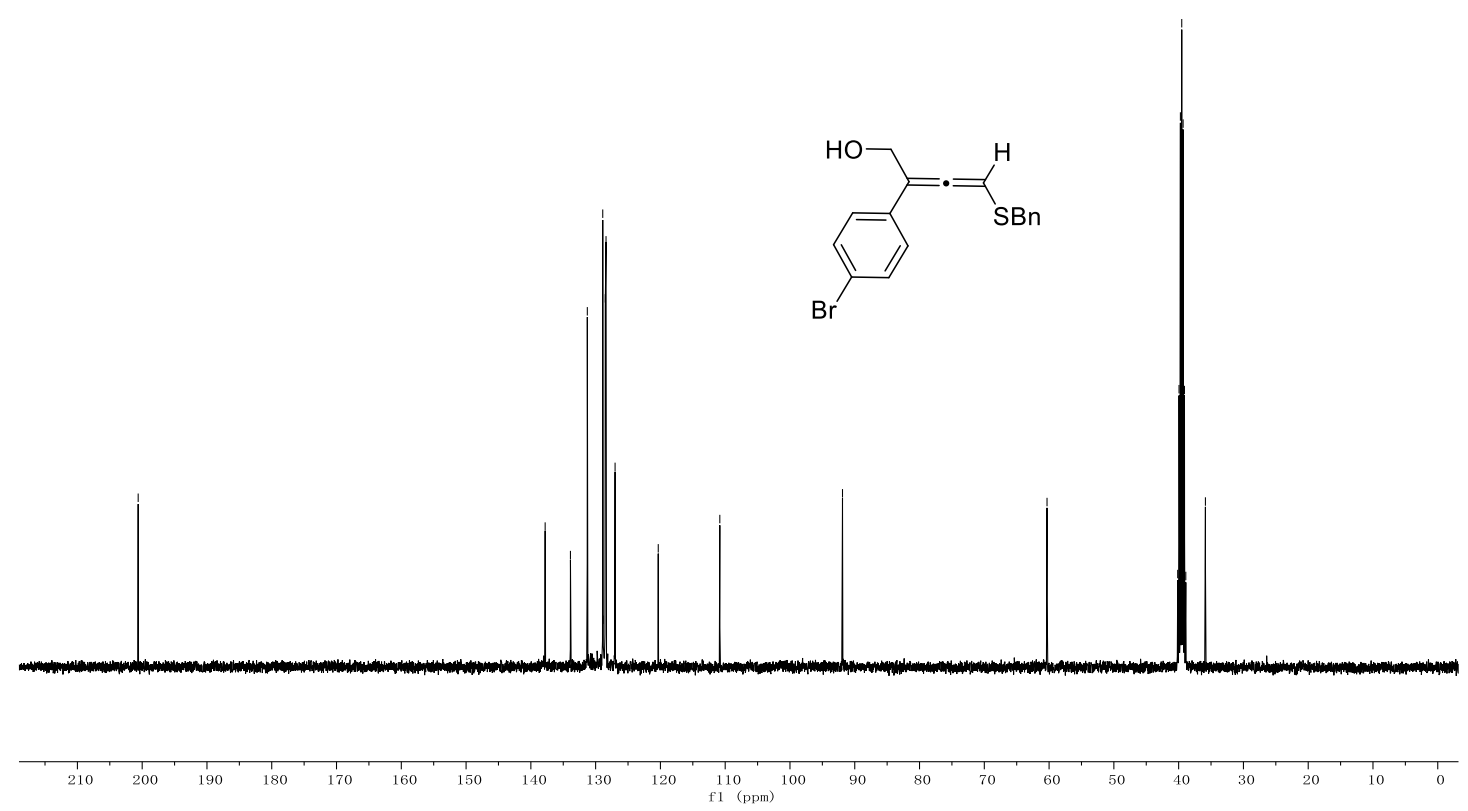


${ }^{1} \mathrm{H}$ NMR $\left(300 \mathrm{MHz}, \mathrm{DMSO}-d_{6}\right)$ of $\mathbf{3 p}$

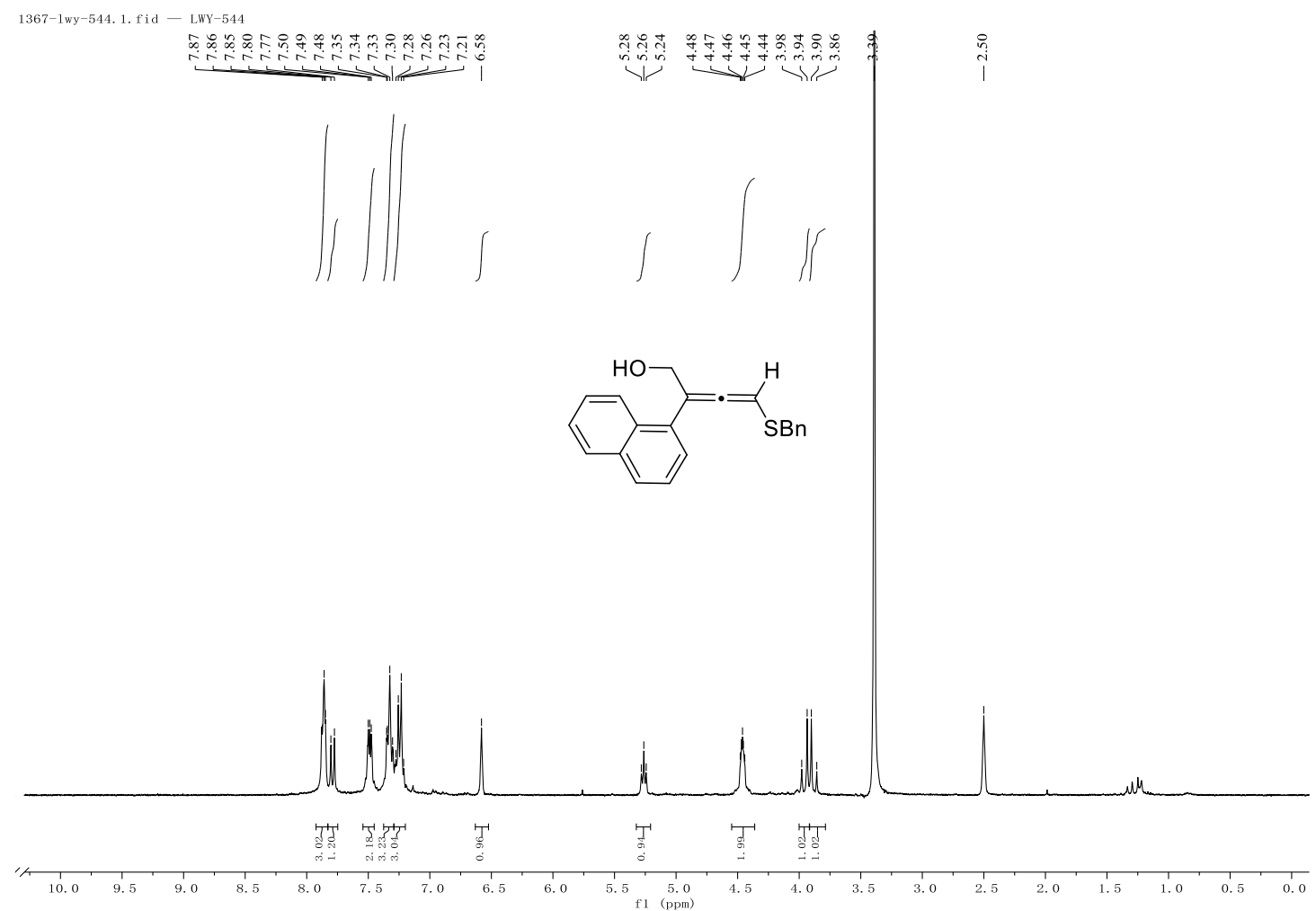

${ }^{13} \mathrm{C}\left\{{ }^{1} \mathrm{H}\right\}$ NMR (101 MHz, DMSO- $\left.d_{6}\right)$ of $\mathbf{3 p}$

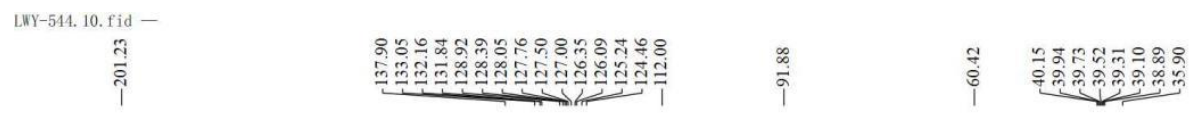
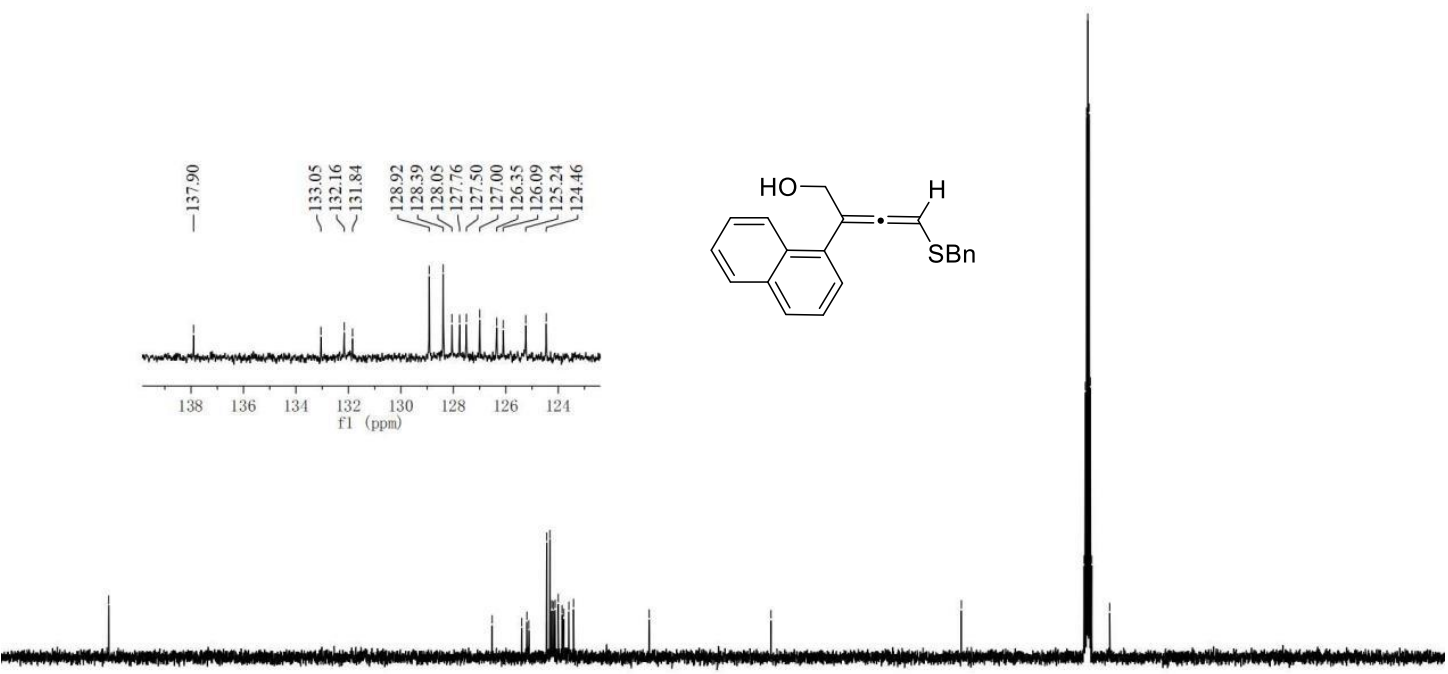

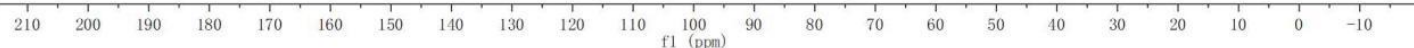


${ }^{1} \mathrm{H}$ NMR (300 MHz, DMSO- $\left.d_{6}\right)$ of 3q

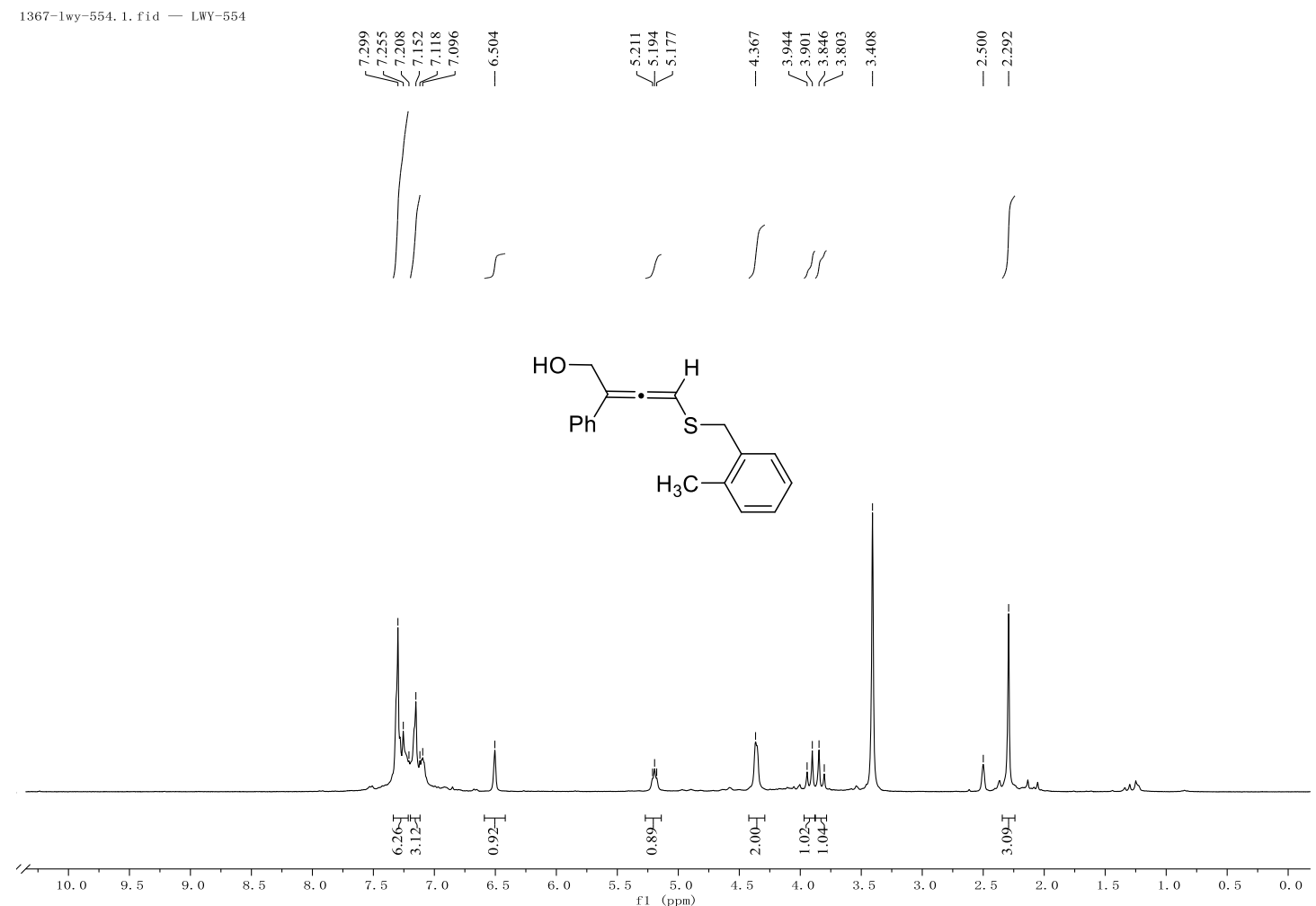

${ }^{13} \mathrm{C}\left\{{ }^{1} \mathrm{H}\right\}$ NMR (300 MHz, DMSO- $d_{6}$ ) of $\mathbf{3 q}$

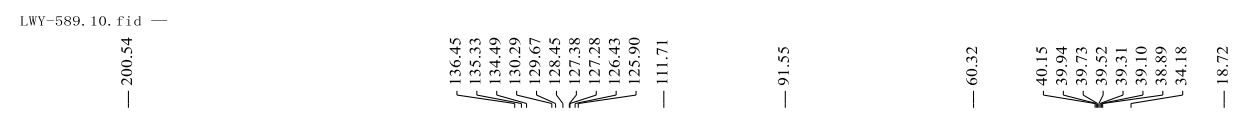
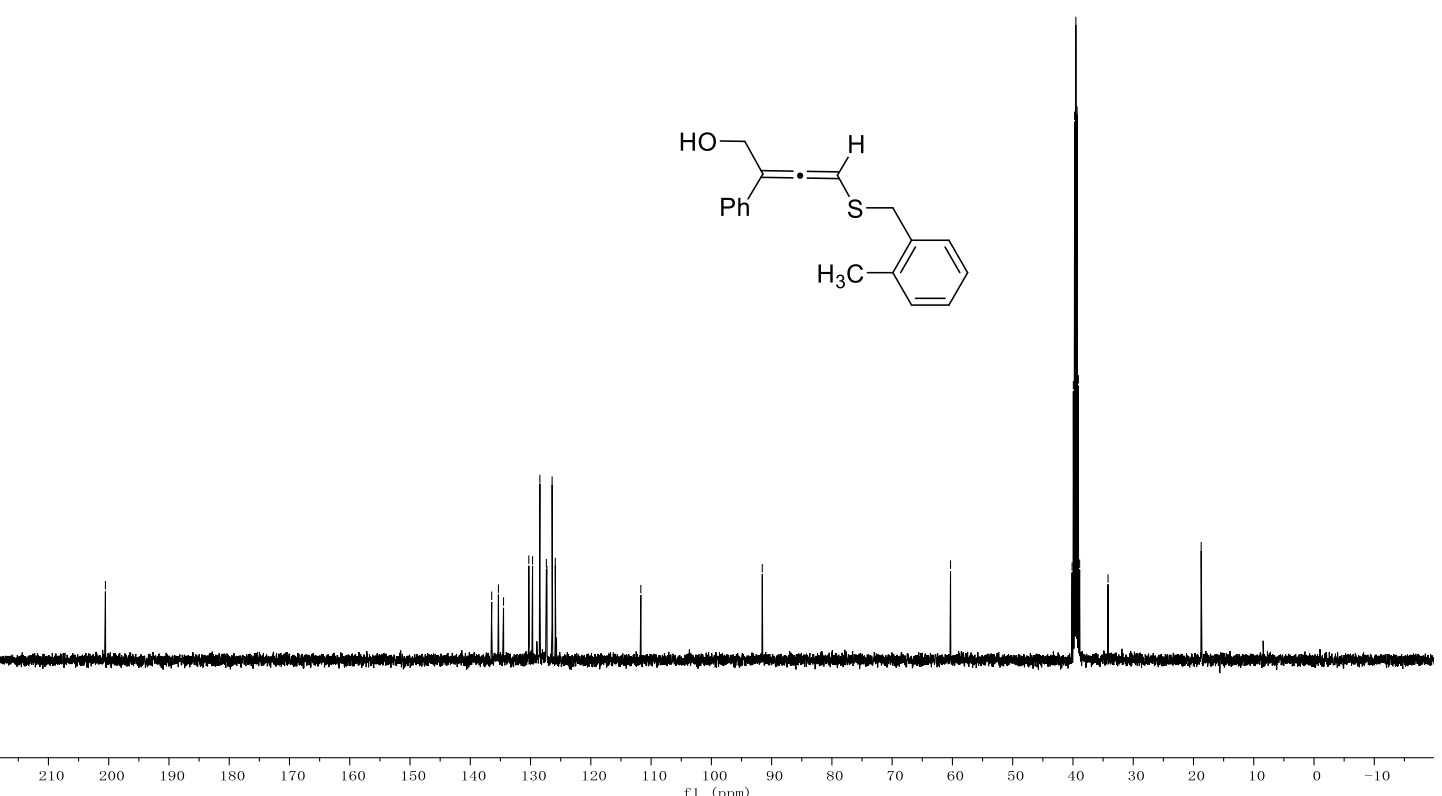
${ }^{1} \mathrm{H}$ NMR (300 MHz, DMSO- $d_{6}$ ) of $\mathbf{3 r}$

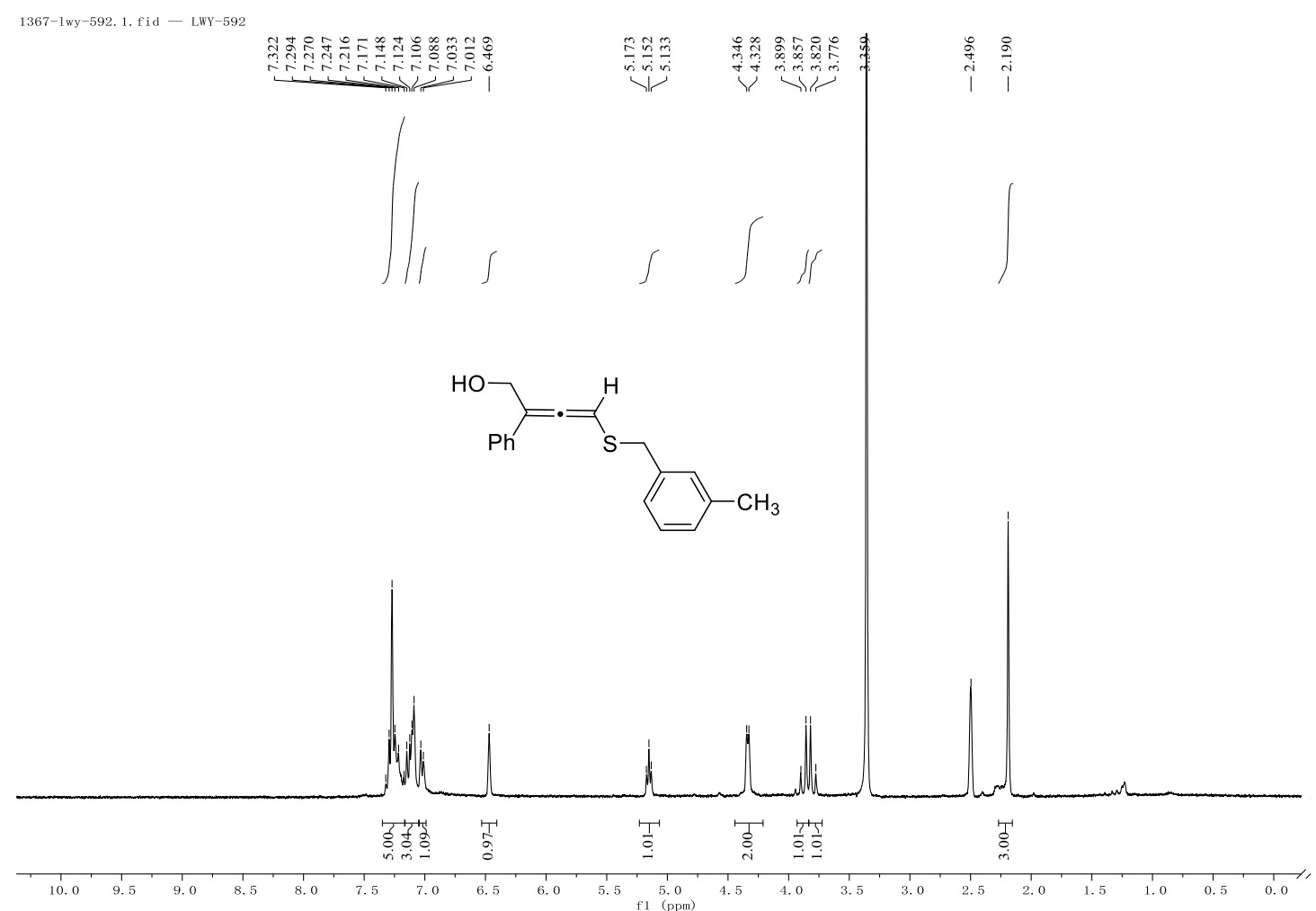

${ }^{13} \mathrm{C}\left\{{ }^{1} \mathrm{H}\right\}$ NMR (101 MHz, DMSO- $d_{6}$ ) of $\mathbf{3 r}$

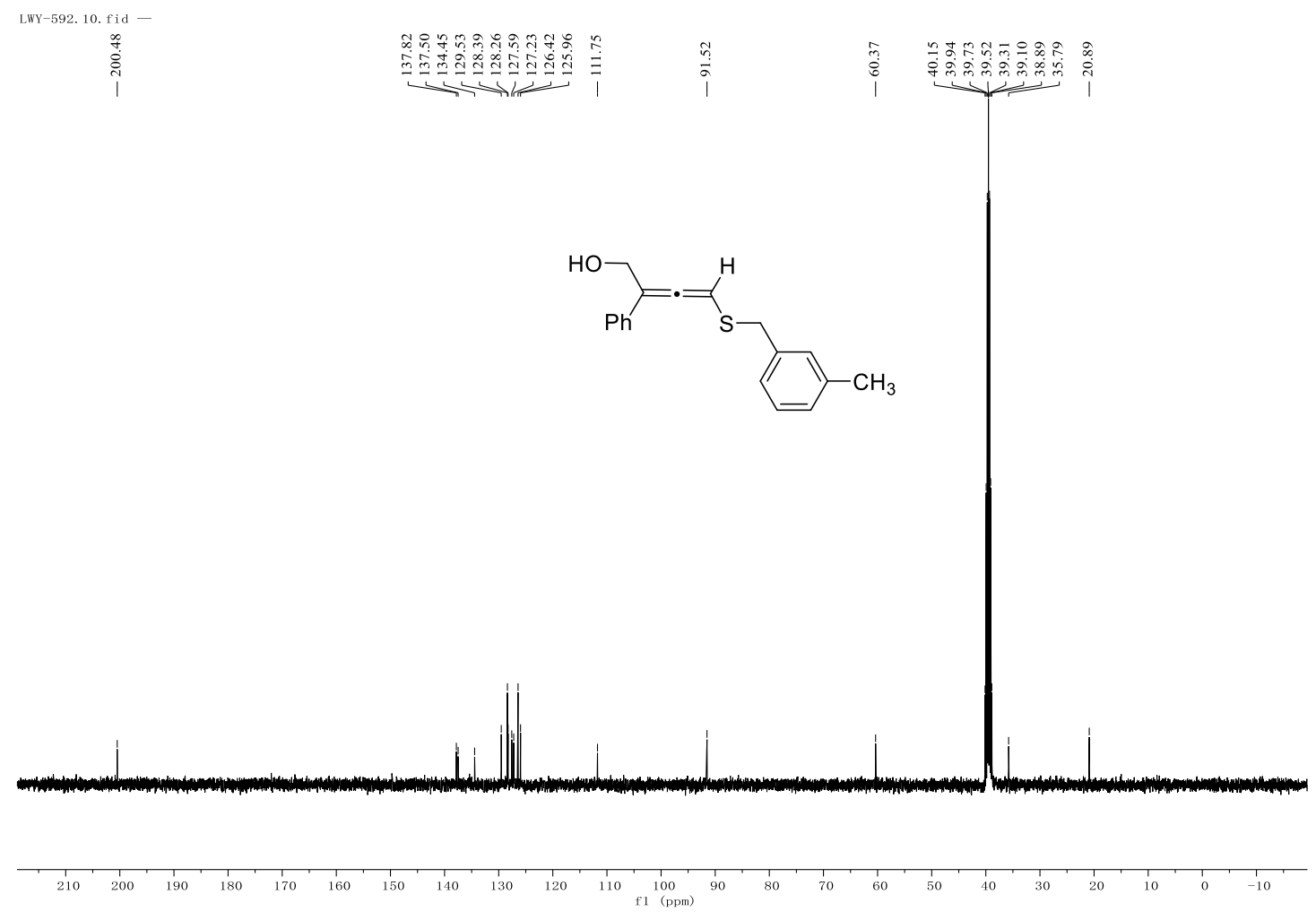


${ }^{1} \mathrm{H}$ NMR $\left(300 \mathrm{MHz}\right.$, DMSO- $d_{6}$ ) of $\mathbf{3 s}$

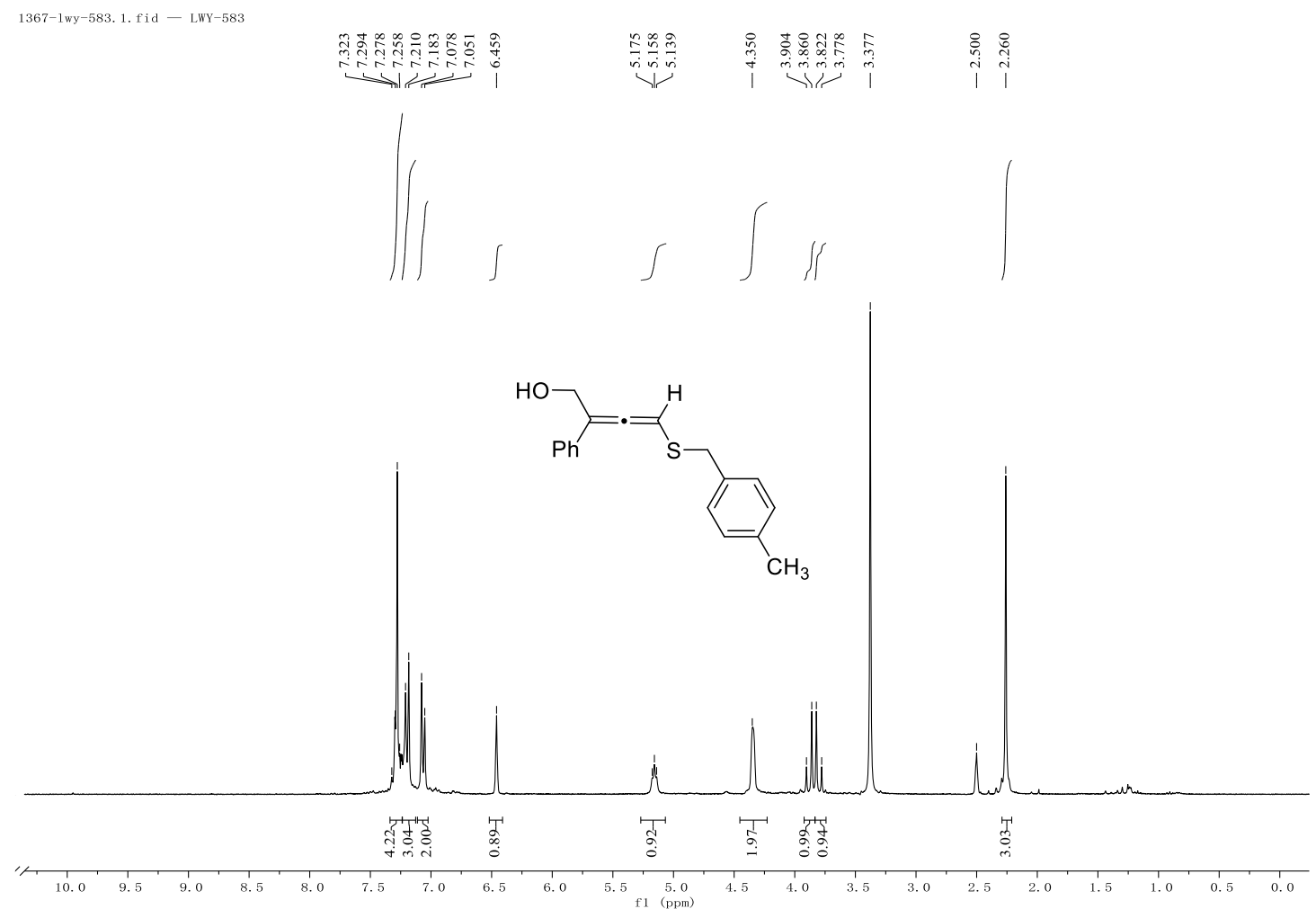

${ }^{13} \mathrm{C}\left\{{ }^{1} \mathrm{H}\right\}$ NMR (151 MHz, DMSO- $\left.d_{6}\right)$ of $\mathbf{3 s}$

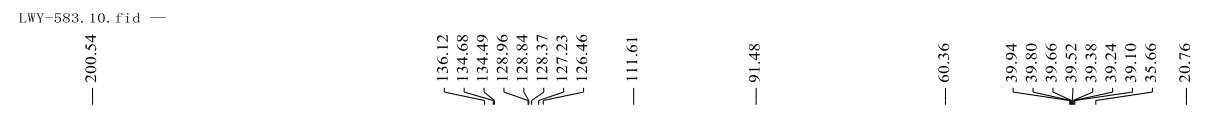
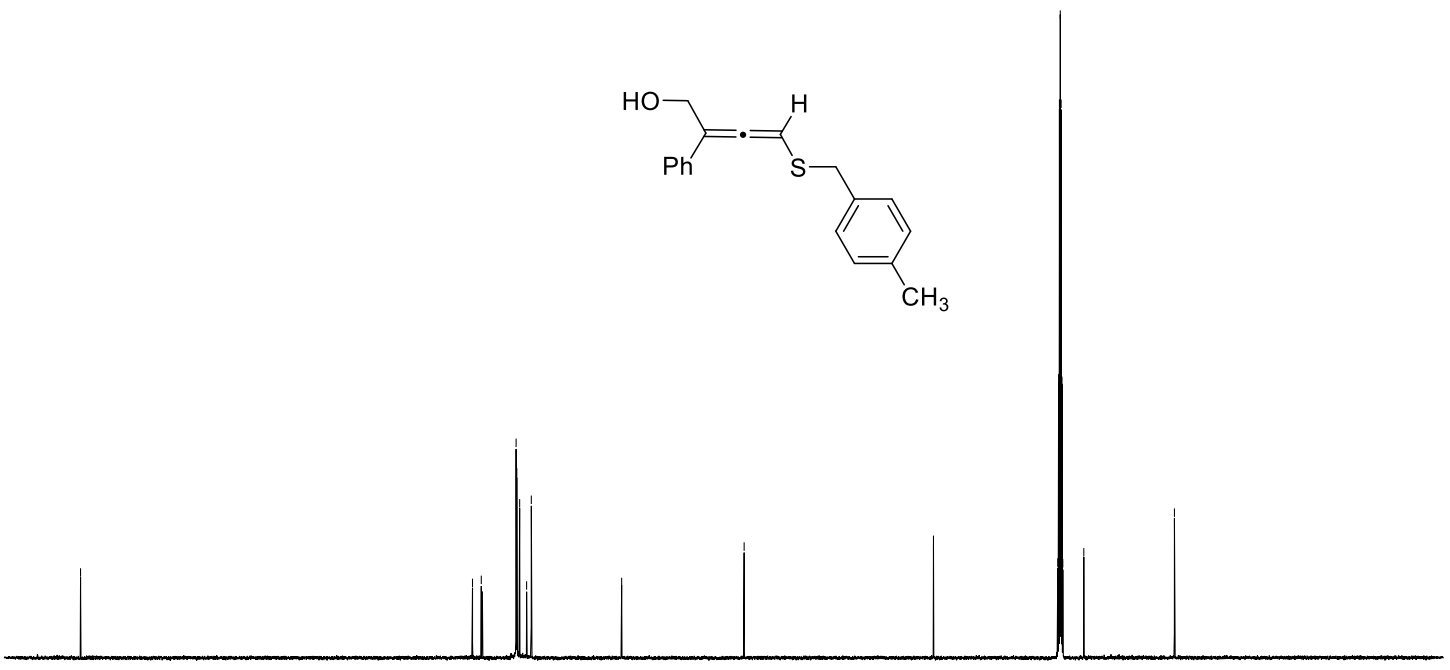

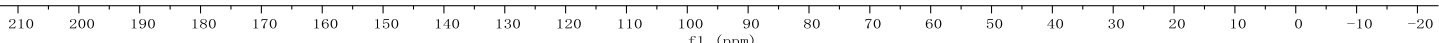


${ }^{1} \mathrm{H}$ NMR (300 MHz, DMSO- $d_{6}$ ) of $\mathbf{3 t}$

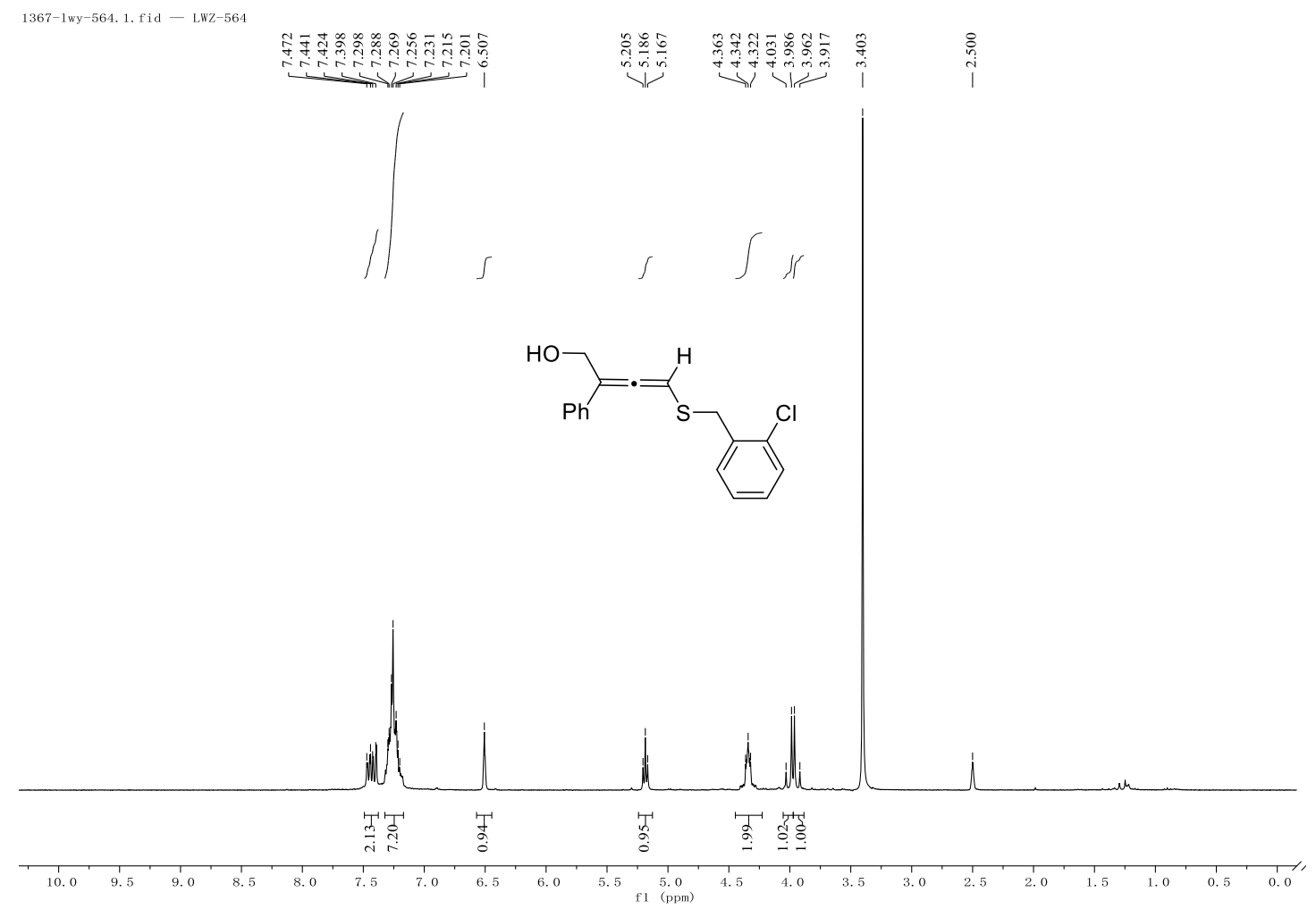

${ }^{13} \mathrm{C}\left\{{ }^{1} \mathrm{H}\right\}$ NMR (101 MHz, DMSO- $\left.d_{6}\right)$ of $3 \mathrm{t}$

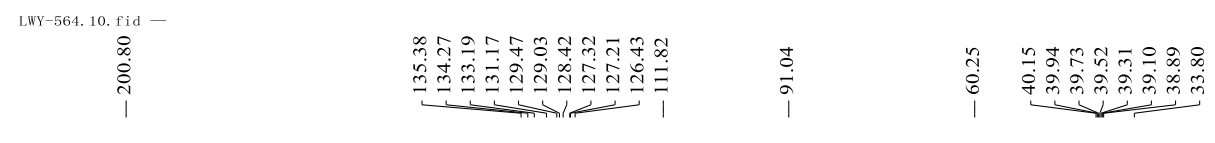
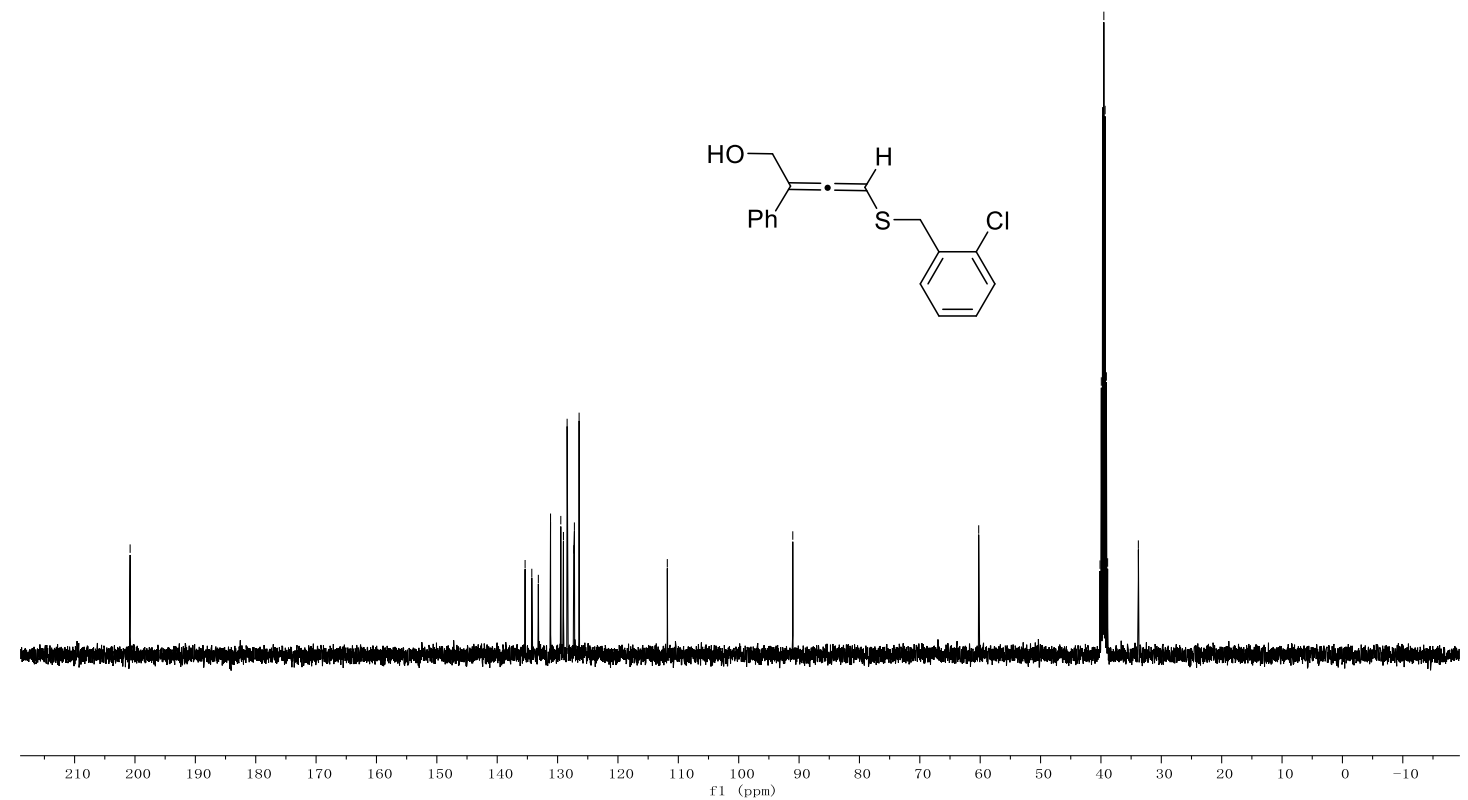
${ }^{1} \mathrm{H}$ NMR (300 MHz, DMSO- $d_{6}$ ) of $\mathbf{3 u}$

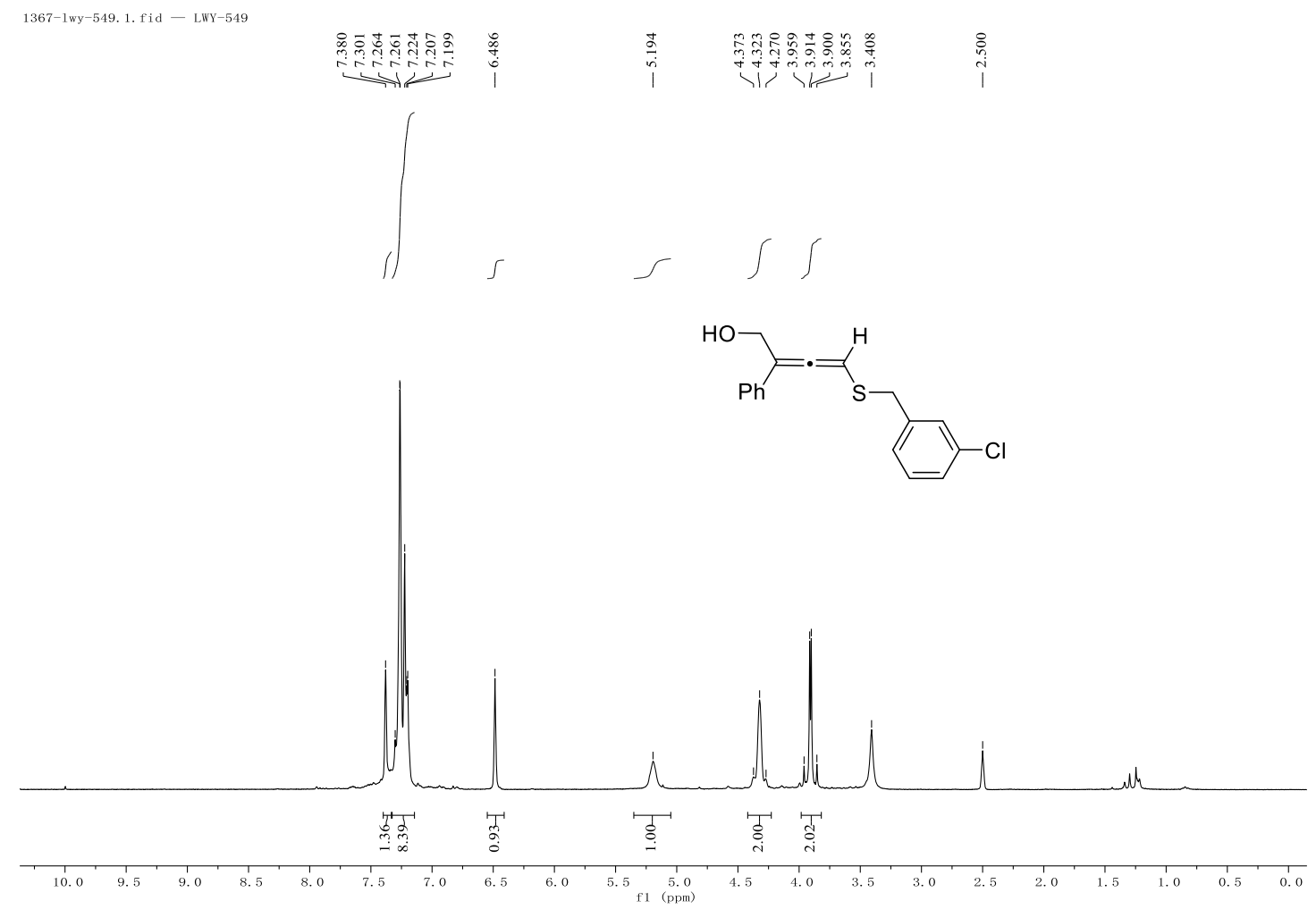

${ }^{13} \mathrm{C}\left\{{ }^{1} \mathrm{H}\right\}$ NMR (101 MHz, DMSO- $\left.d_{6}\right)$ of $\mathbf{3 u}$

\begin{tabular}{|c|c|c|c|c|}
\hline $\begin{array}{l}\infty \\
\infty \\
\stackrel{0}{0} \\
1\end{array}$ & 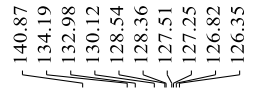 & $\frac{8}{i}$ & $\begin{array}{c}\text { ते } \\
\stackrel{0}{0} \\
1\end{array}$ & 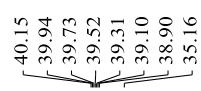 \\
\hline
\end{tabular}
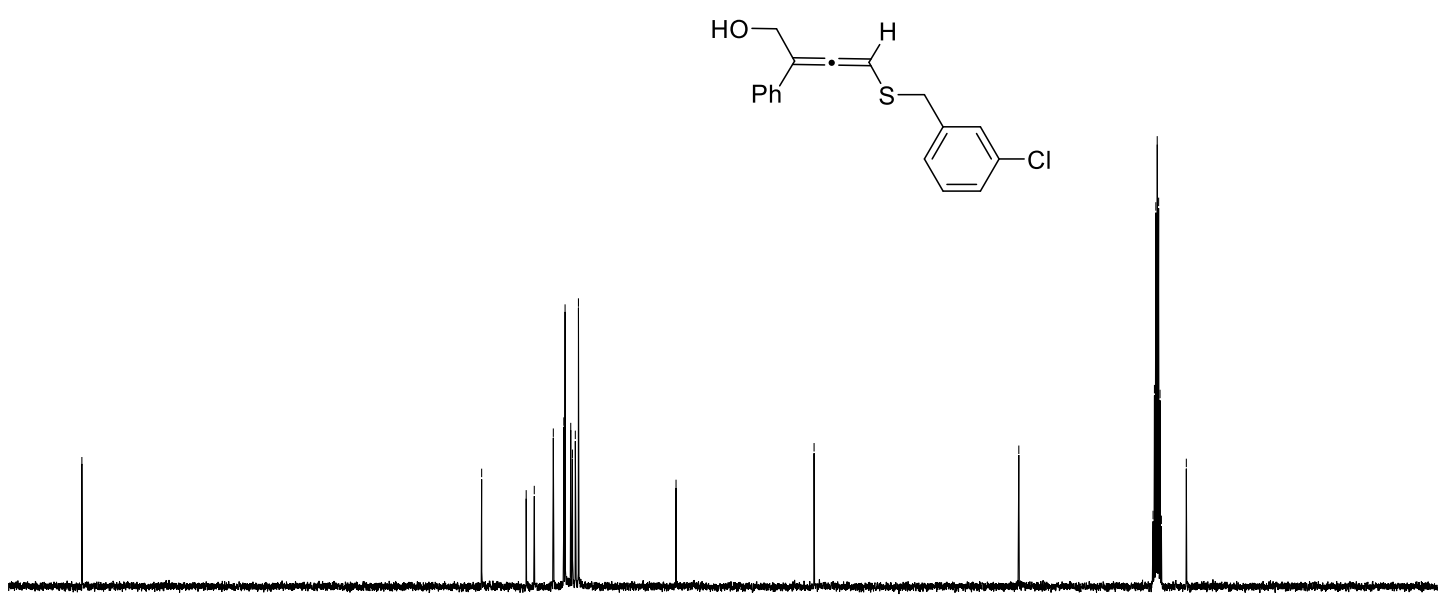

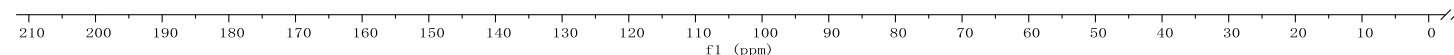


${ }^{1} \mathrm{H}$ NMR (300 MHz, DMSO- $d_{6}$ ) of $\mathbf{3 v}$

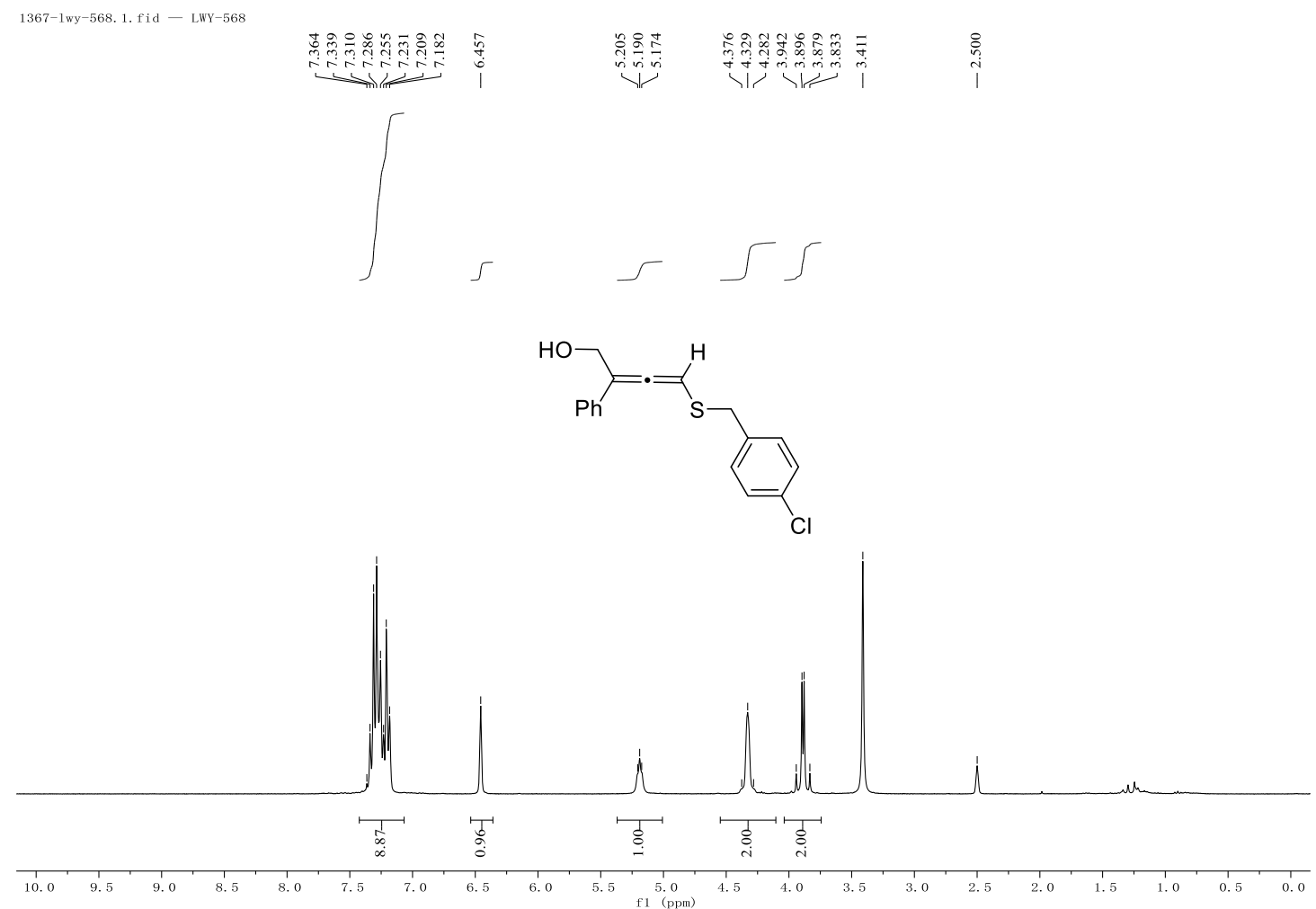

${ }^{13} \mathrm{C}\left\{{ }^{1} \mathrm{H}\right\}$ NMR (101 MHz, DMSO- $\left.d_{6}\right)$ of $\mathbf{3 v}$

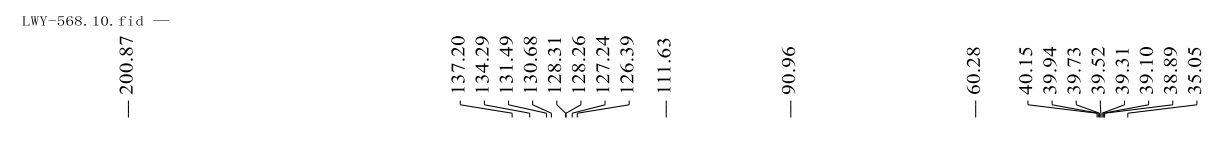
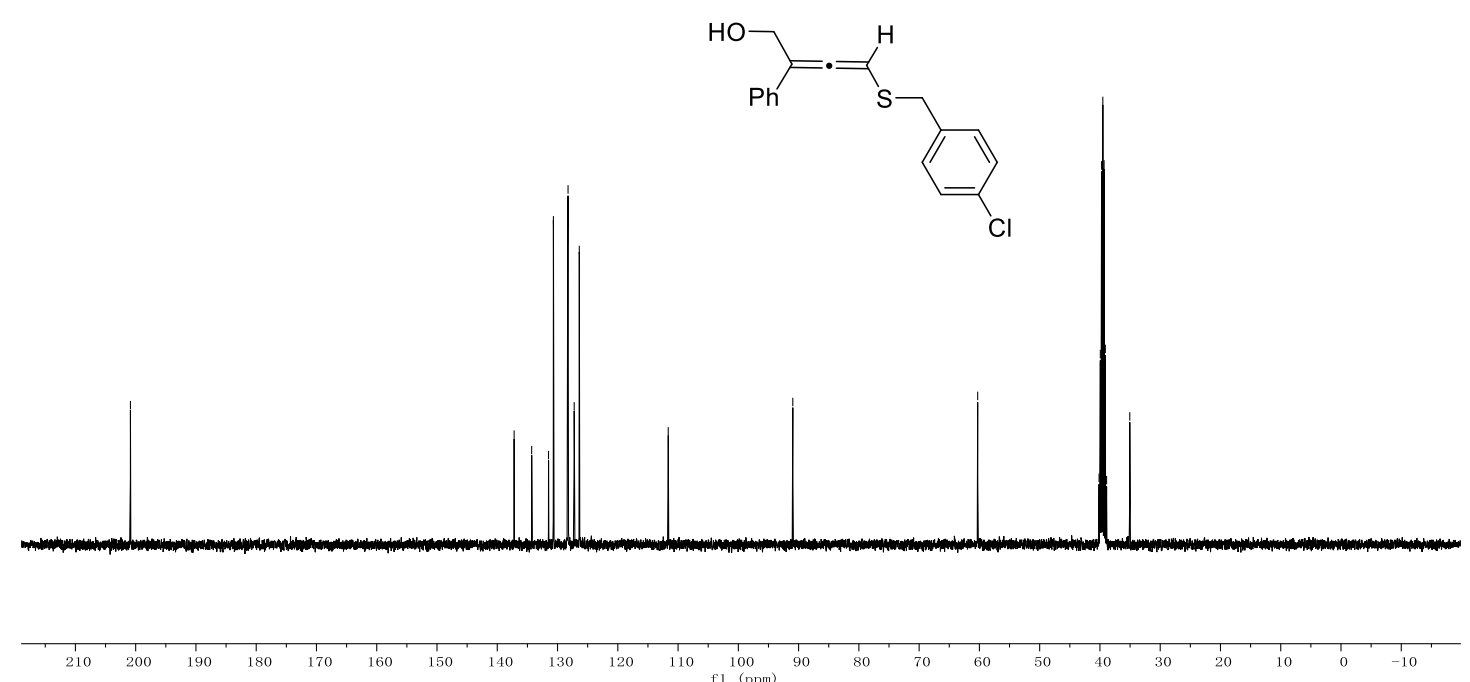
${ }^{1} \mathrm{H}$ NMR (300 MHz, DMSO- $\left.d_{6}\right)$ of $\mathbf{3 w}$

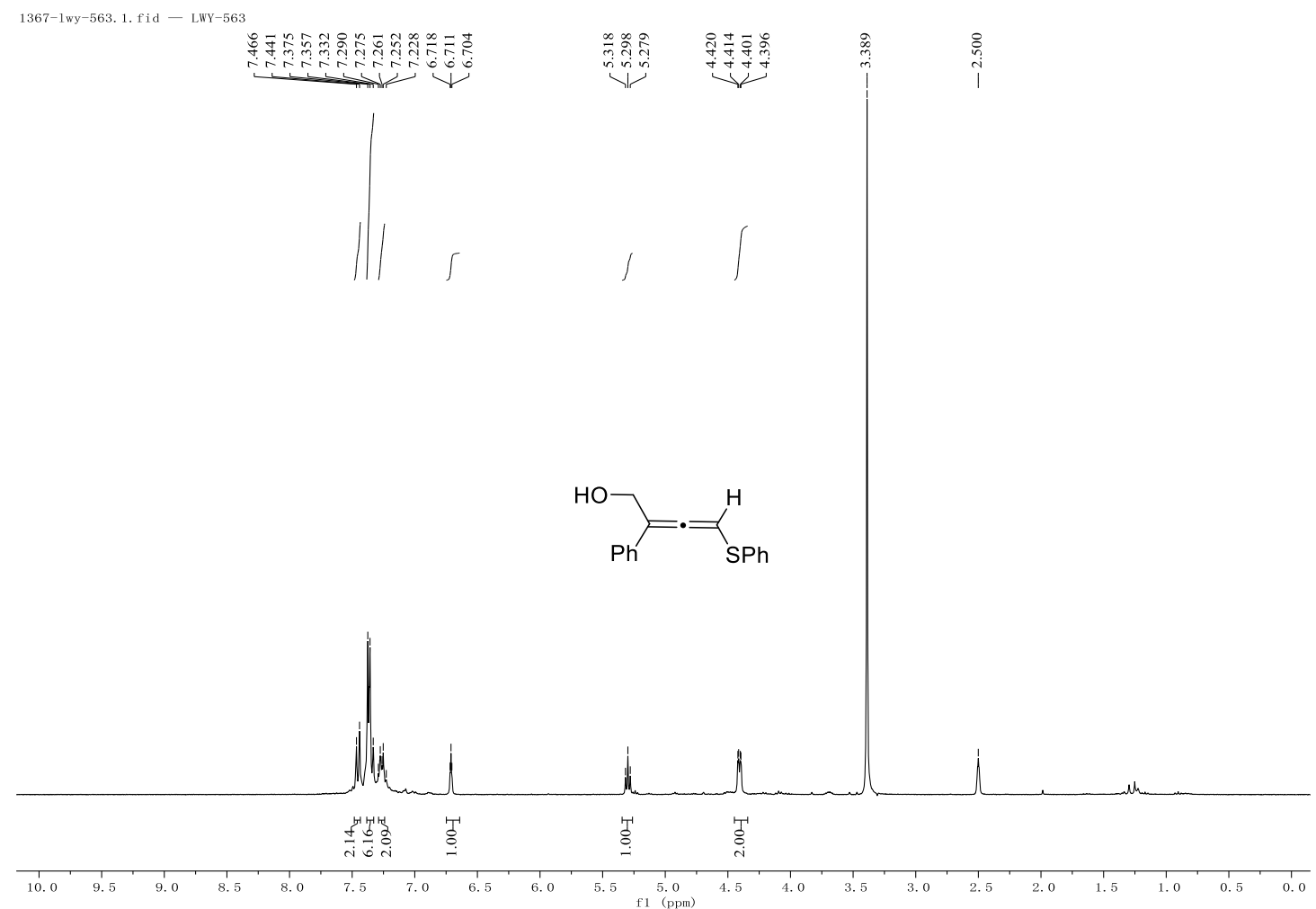

${ }^{13} \mathrm{C}\left\{{ }^{1} \mathrm{H}\right\}$ NMR (101 MHz, DMSO- $\left.d_{6}\right)$ of $\mathbf{3 w}$

\begin{tabular}{|c|c|c|c|c|}
\hline $\begin{array}{l}\infty \\
\stackrel{\infty}{0} \\
\stackrel{\mathrm{N}}{1}\end{array}$ & 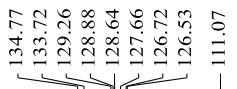 & 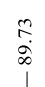 & $\begin{array}{l}\infty \\
\infty \\
\infty \\
\infty \\
1\end{array}$ & 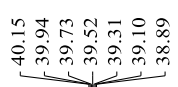 \\
\hline
\end{tabular}

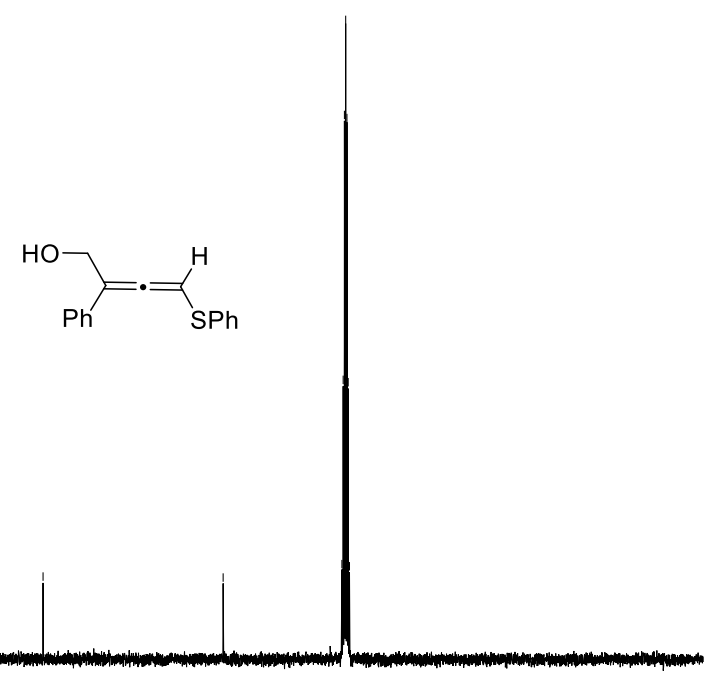

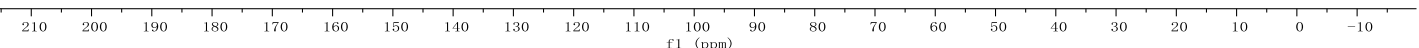


${ }^{1} \mathrm{H}$ NMR (300 MHz, DMSO- $d_{6}$ ) of $\mathbf{3 x}$

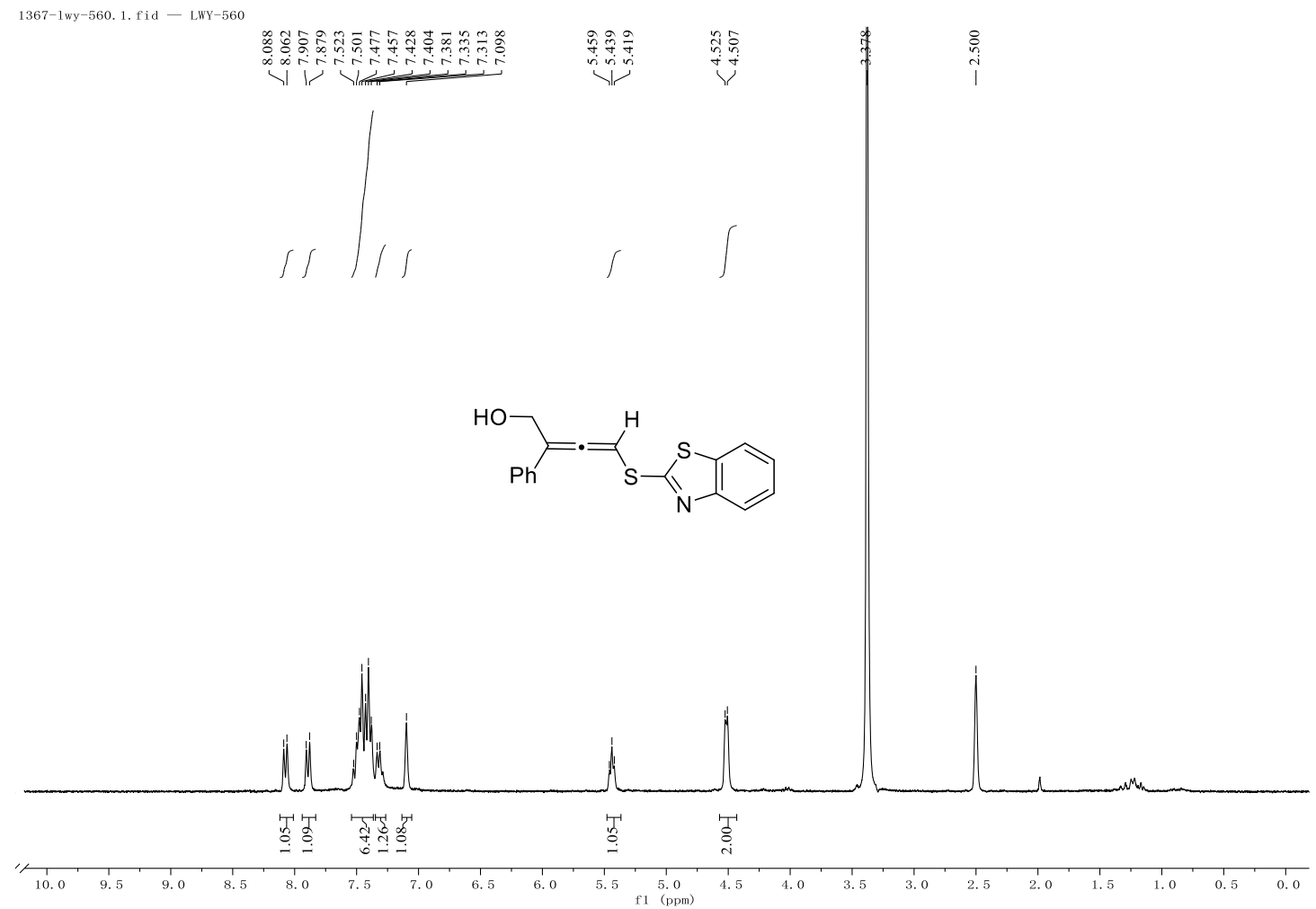

${ }^{13} \mathrm{C}\left\{{ }^{1} \mathrm{H}\right\}$ NMR (101 MHz, DMSO- $\left.d_{6}\right)$ of $\mathbf{3 x}$
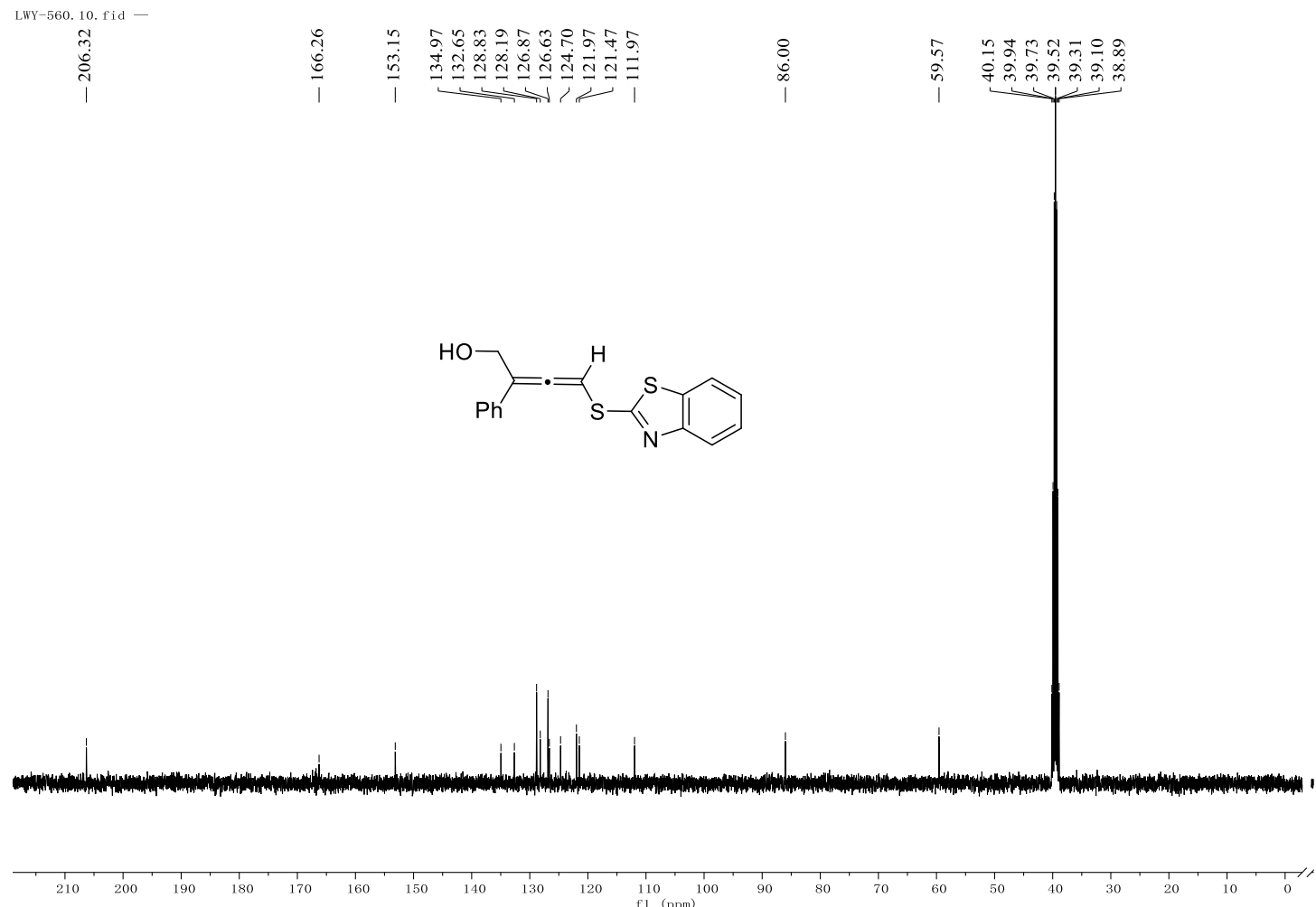
${ }^{1} \mathrm{H}$ NMR (400 MHz, DMSO- $d_{6}$ ) of $\mathbf{3 y}$
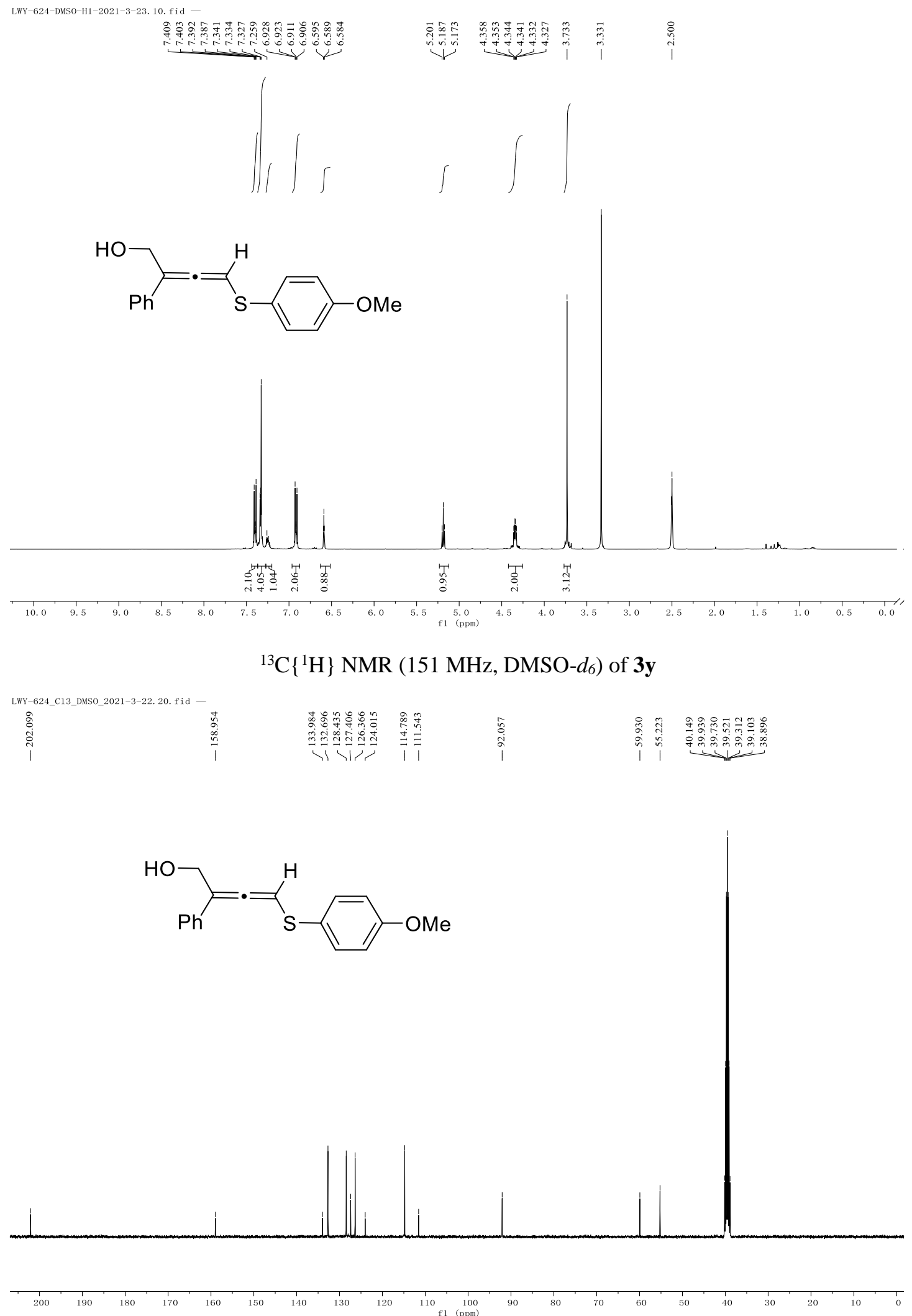
${ }^{1} \mathrm{H}$ NMR (300 MHz, DMSO- $d_{6}$ ) of $\mathbf{3 z}$

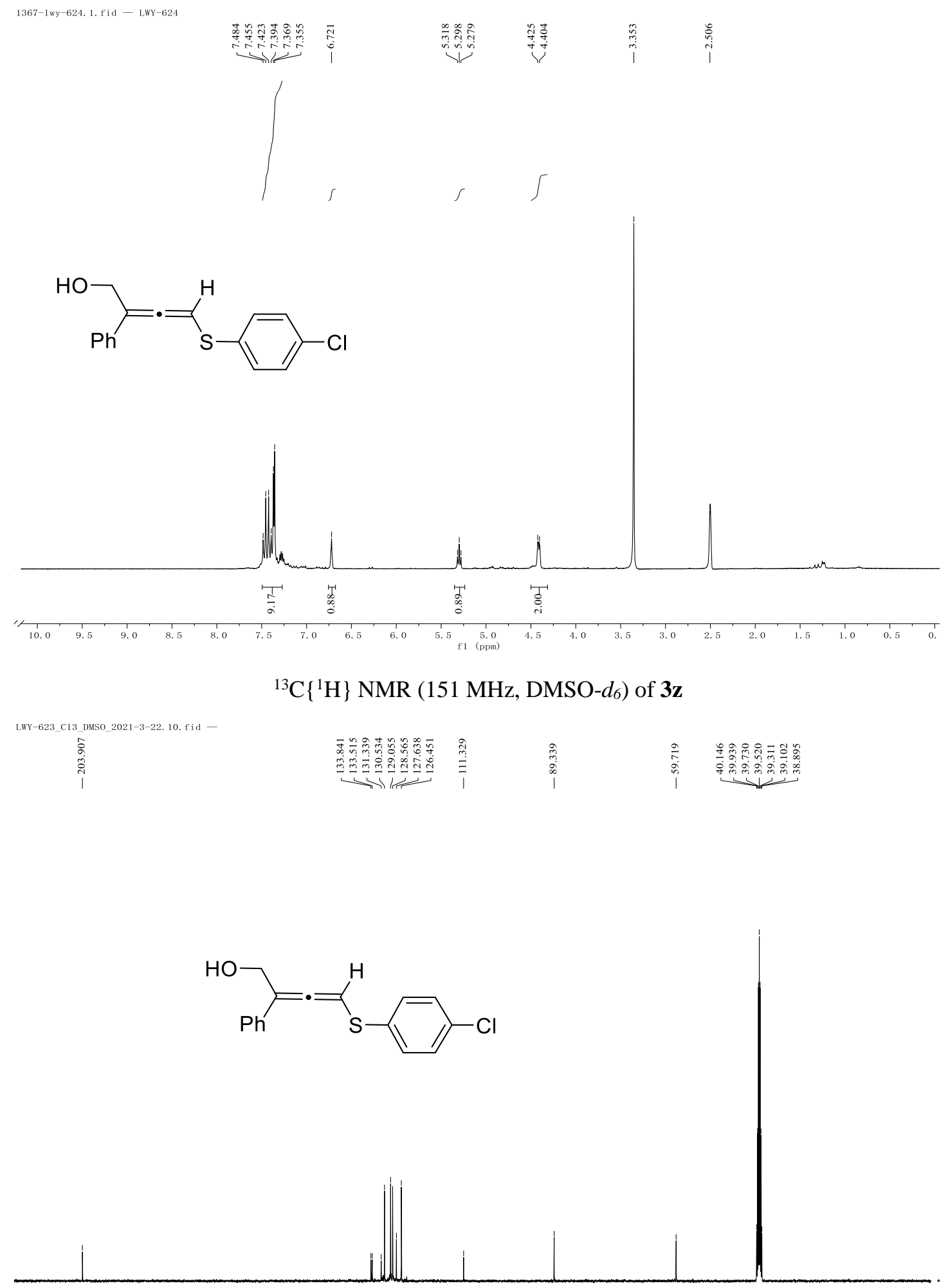

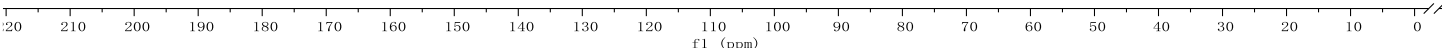


${ }^{1} \mathrm{H}$ NMR (300 MHz, DMSO- $d_{6}$ ) of 3a'

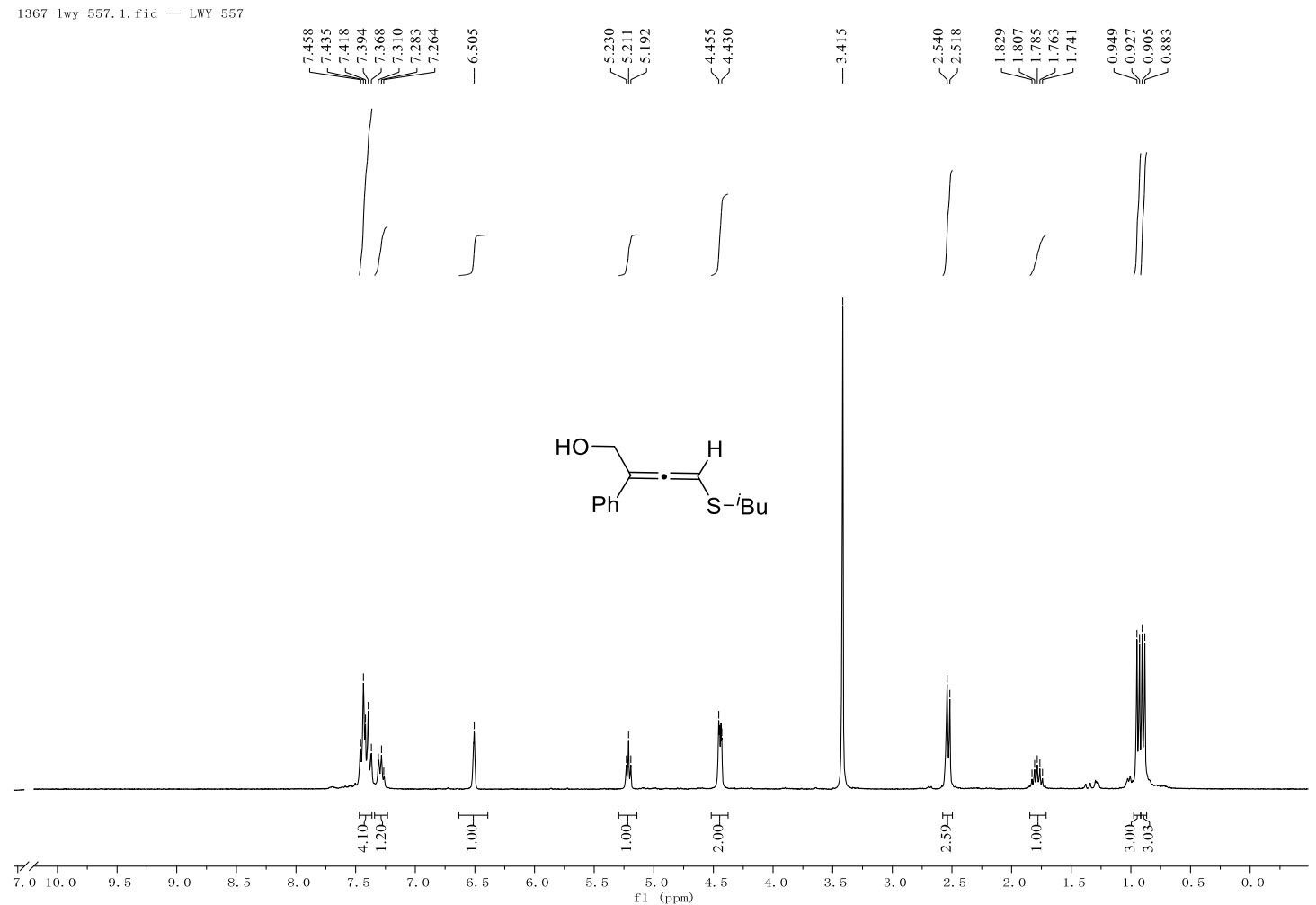

${ }^{13} \mathrm{C}\left\{{ }^{1} \mathrm{H}\right\}$ NMR (101 MHz, DMSO- $\left.d_{6}\right)$ of $\mathbf{3 a} \mathbf{a}^{\prime}$

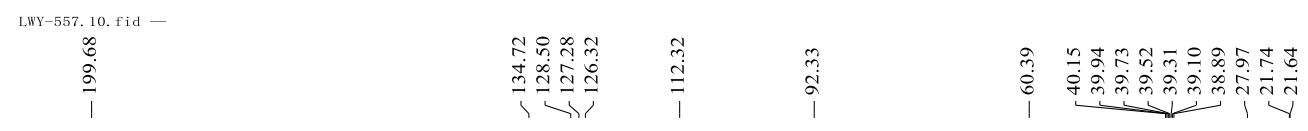

$\underset{\mathrm{Ph}}{\mathrm{HO}}=\mathrm{S}_{\mathrm{S}-{ }^{-} \mathrm{Bu}}^{\mathrm{H}}$

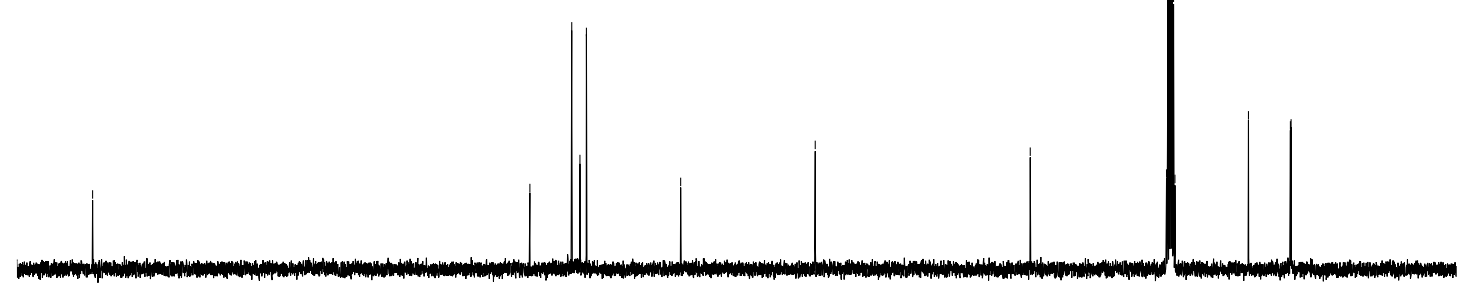

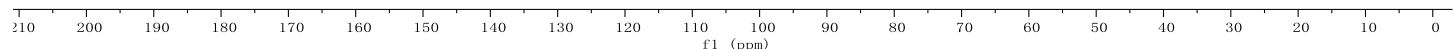


${ }^{1} \mathrm{H}$ NMR (300 MHz, DMSO- $\left.d_{6}\right)$ of $\mathbf{3 b}$

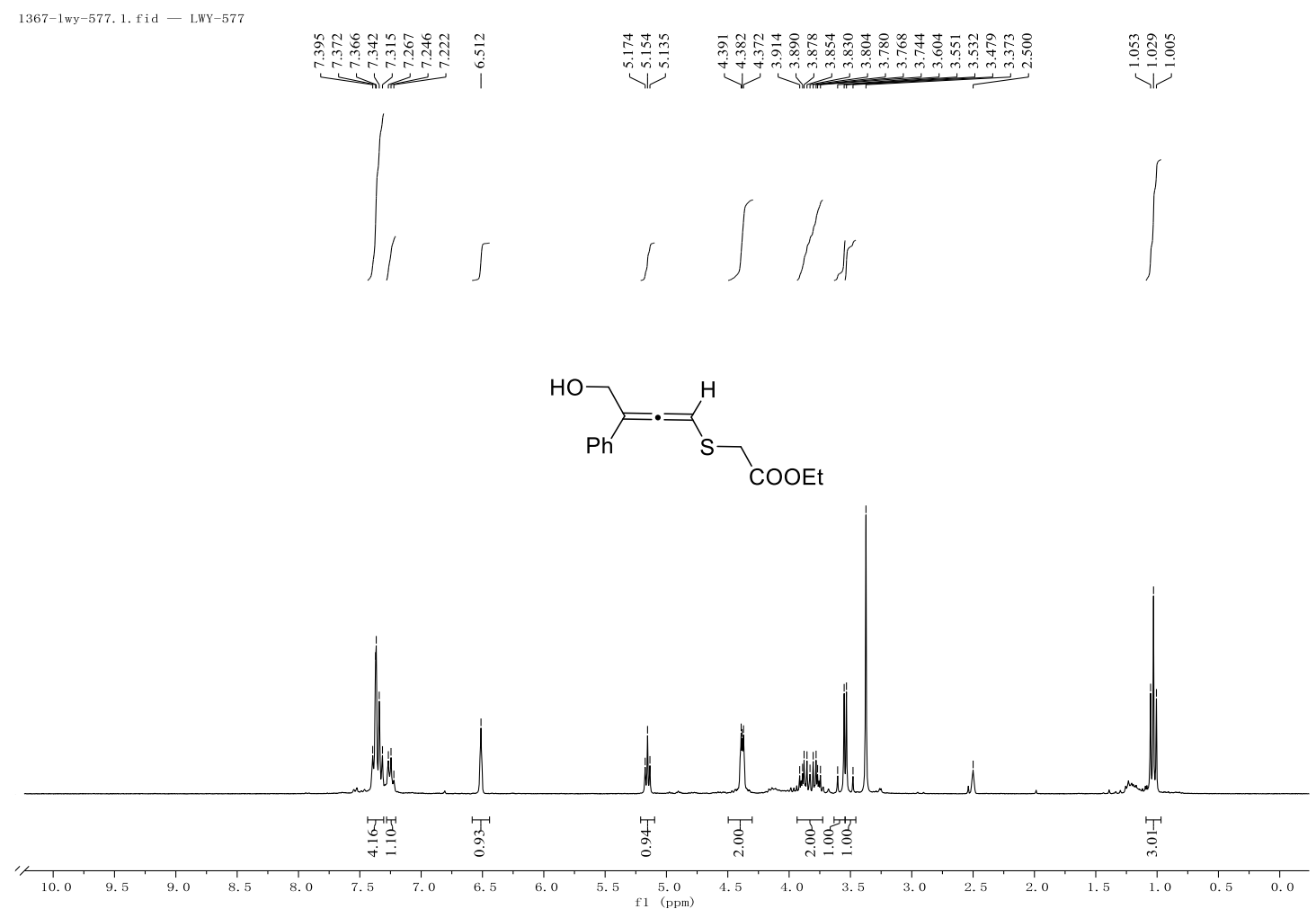

${ }^{13} \mathrm{C}\left\{{ }^{1} \mathrm{H}\right\}$ NMR (151 MHz, DMSO- $\left.d_{6}\right)$ of $\mathbf{3} \mathbf{b}^{\prime}$

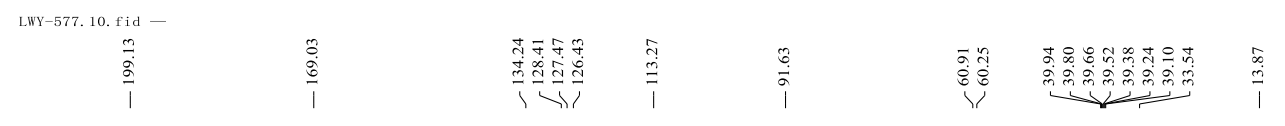

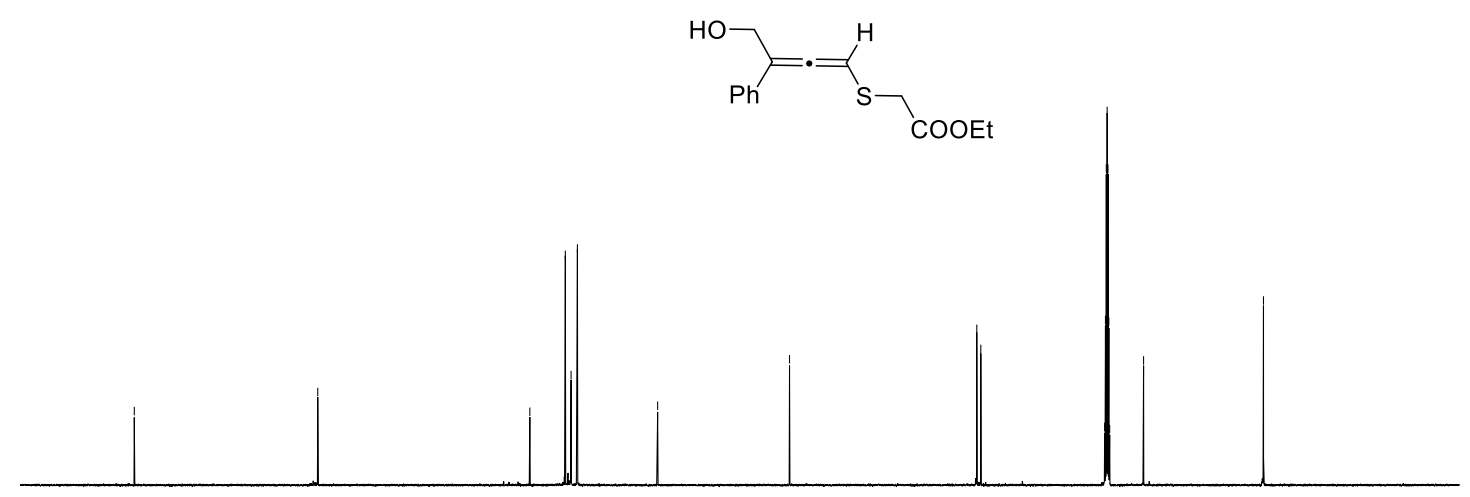

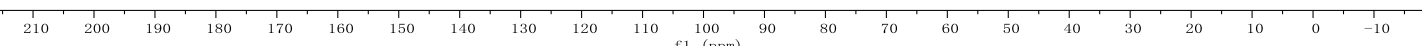


${ }^{1} \mathrm{H}$ NMR (300 MHz, DMSO- $d_{6}$ ) of $\mathbf{5 a}$

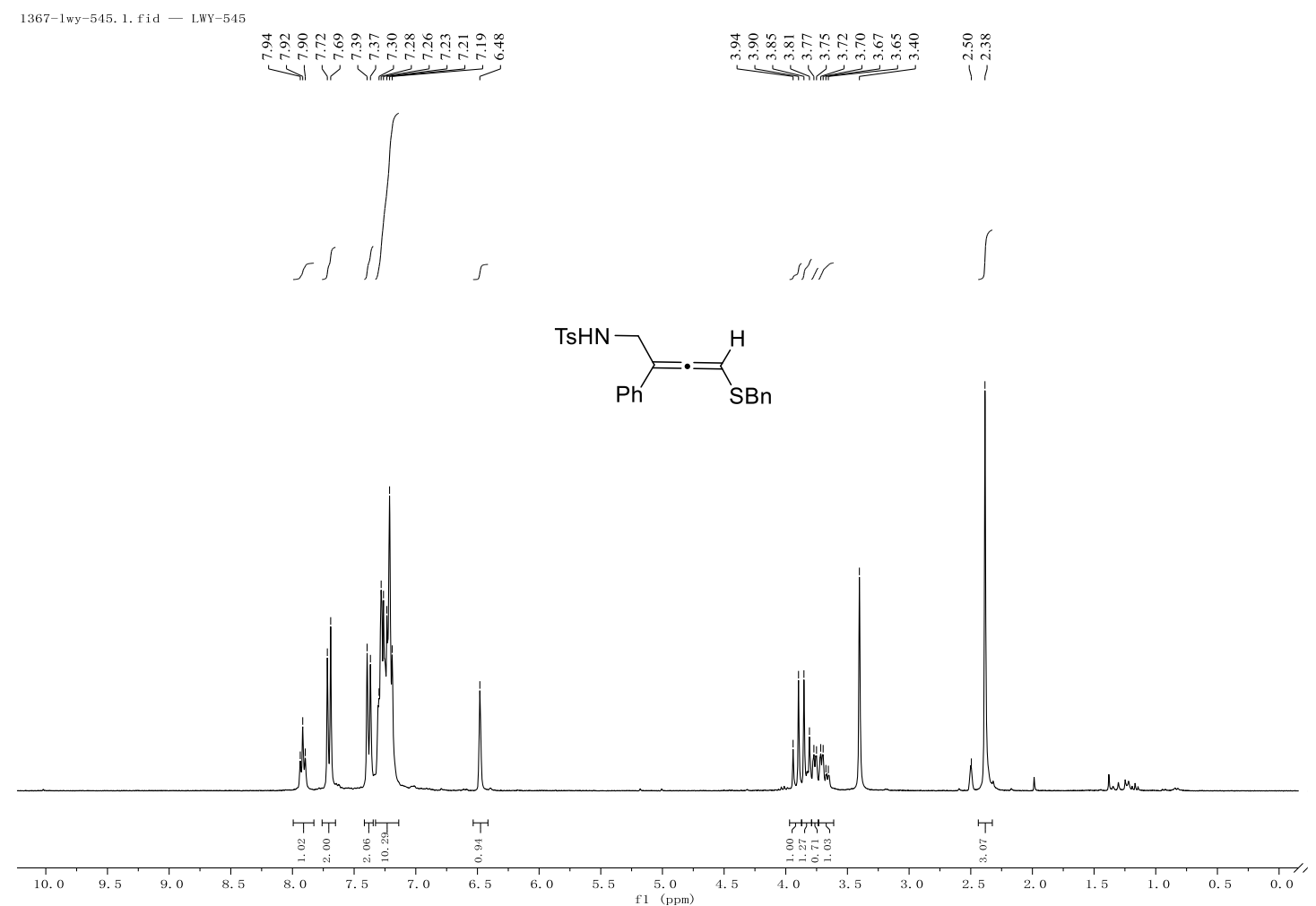

${ }^{13} \mathrm{C}\left\{{ }^{1} \mathrm{H}\right\}$ NMR (101 MHz, DMSO- $\left.d_{6}\right)$ of $5 \mathbf{a}$

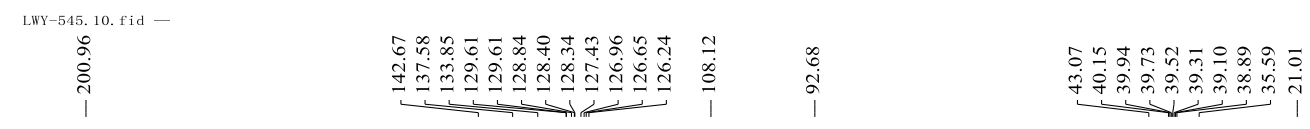

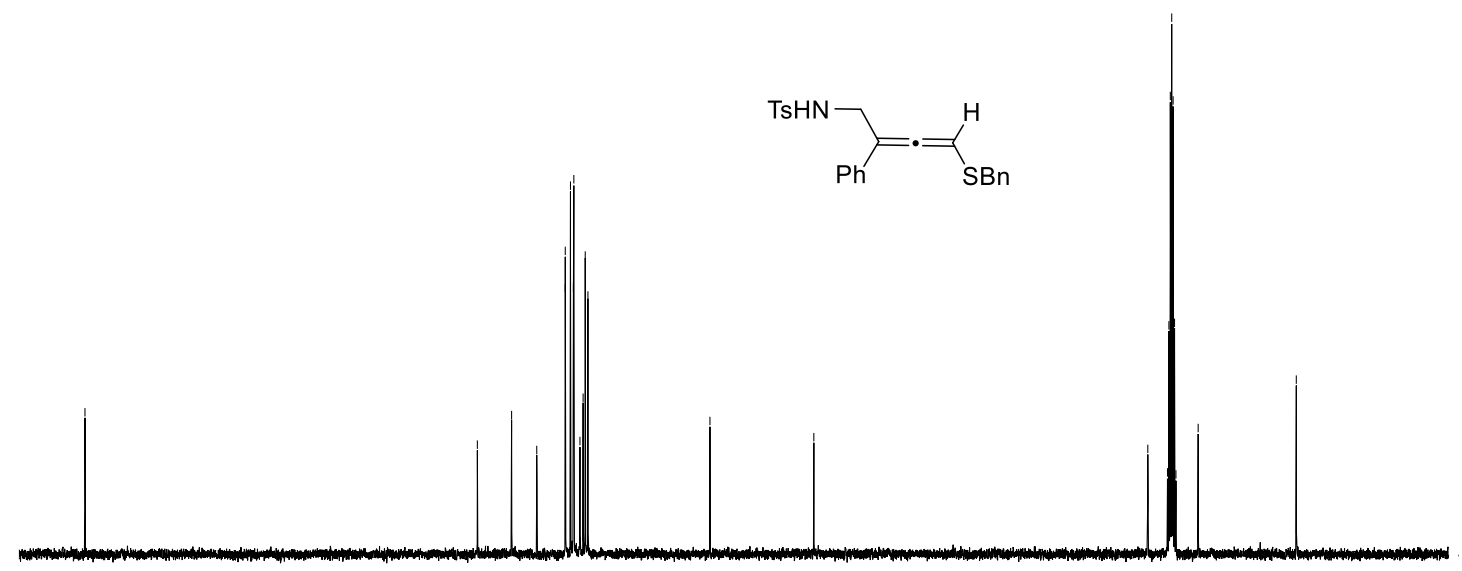

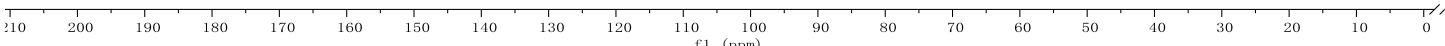


${ }^{1} \mathrm{H}$ NMR (300 MHz, DMSO- $\left.d_{6}\right)$ of $\mathbf{5 b}$

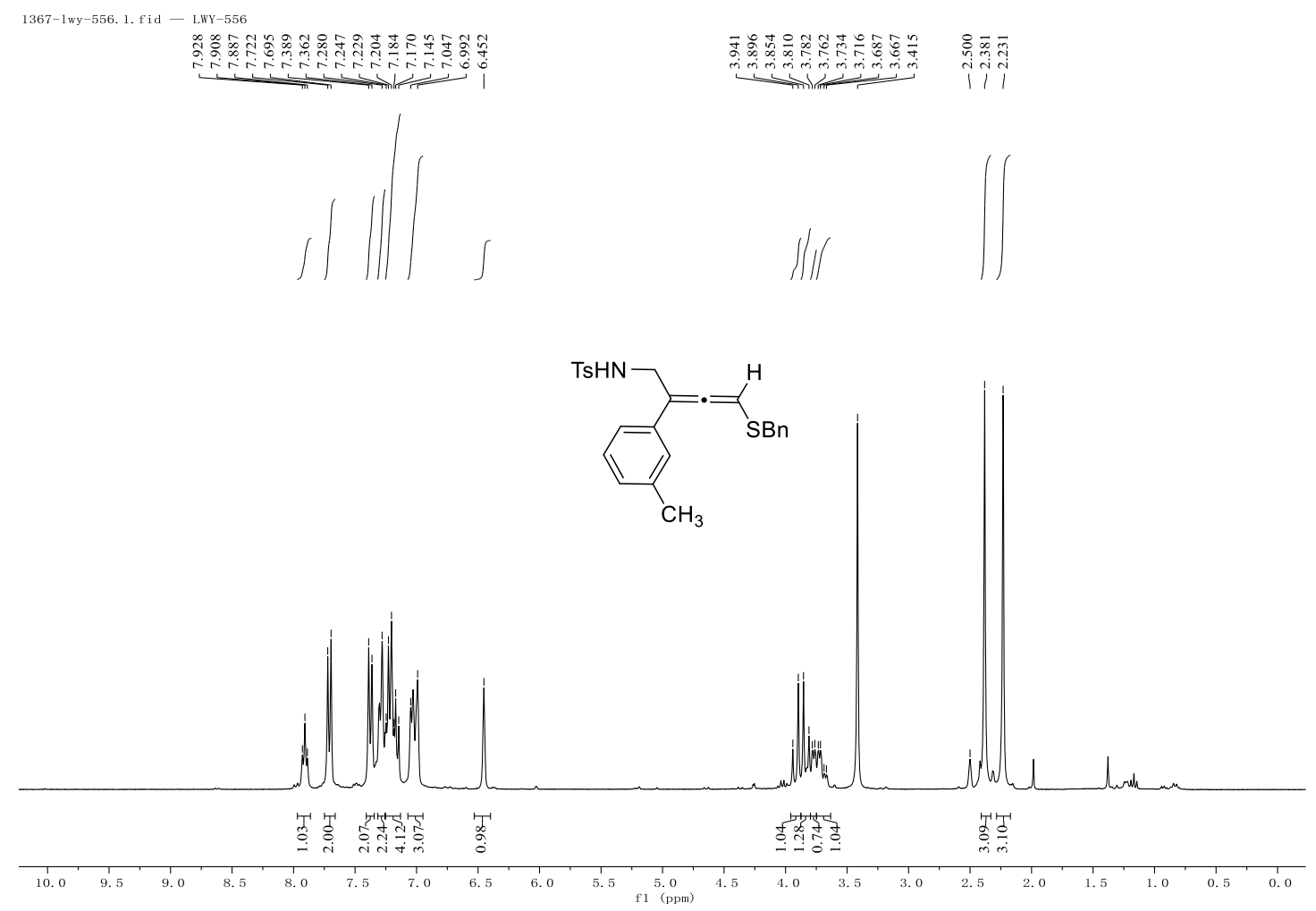

${ }^{13} \mathrm{C}\left\{{ }^{1} \mathrm{H}\right\}$ NMR (101 MHz, DMSO- $\left.d_{6}\right)$ of $\mathbf{5 b}$
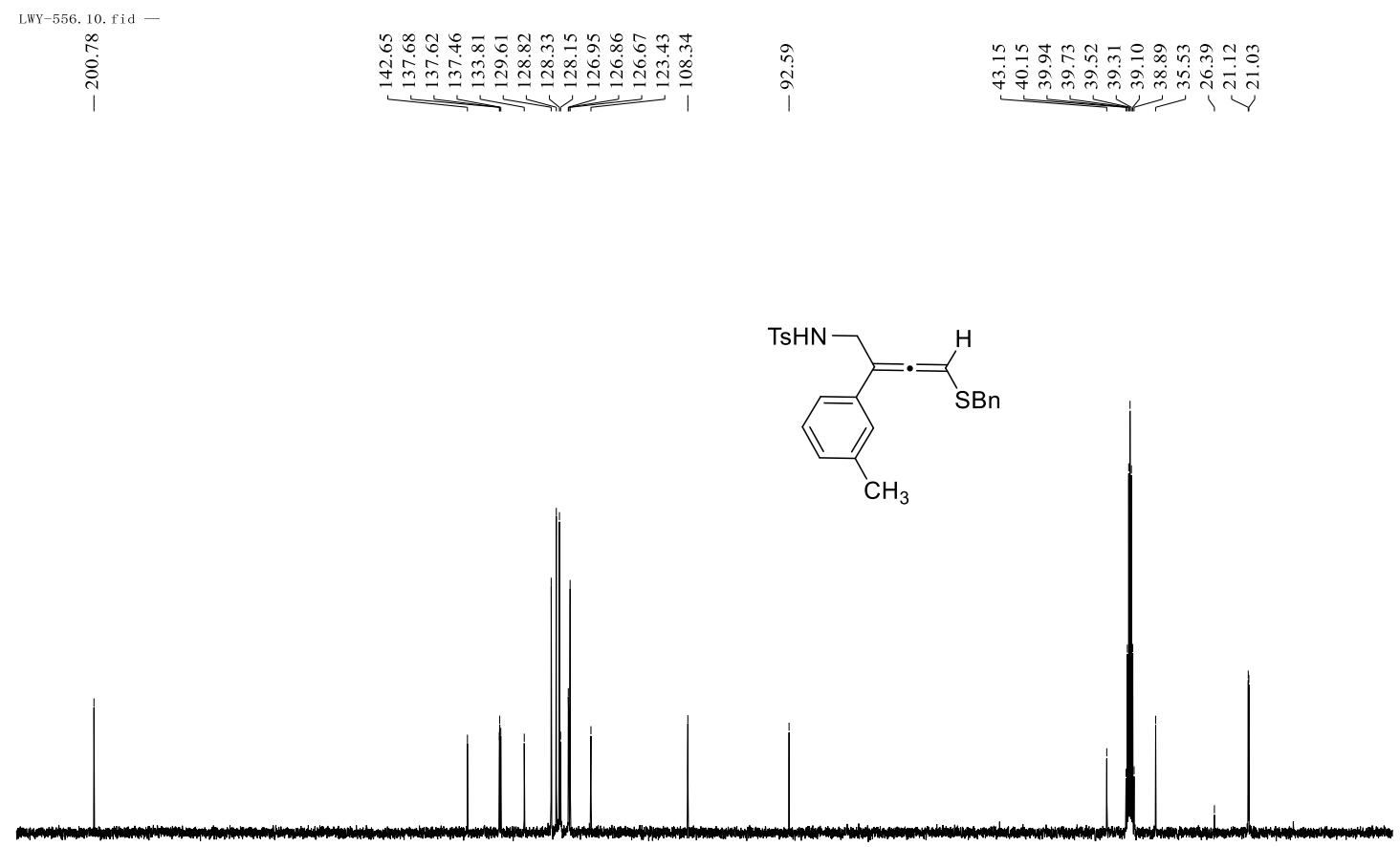

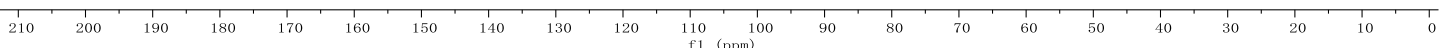


${ }^{1} \mathrm{H}$ NMR (300 MHz, DMSO- $d_{6}$ ) of $\mathbf{5 c}$

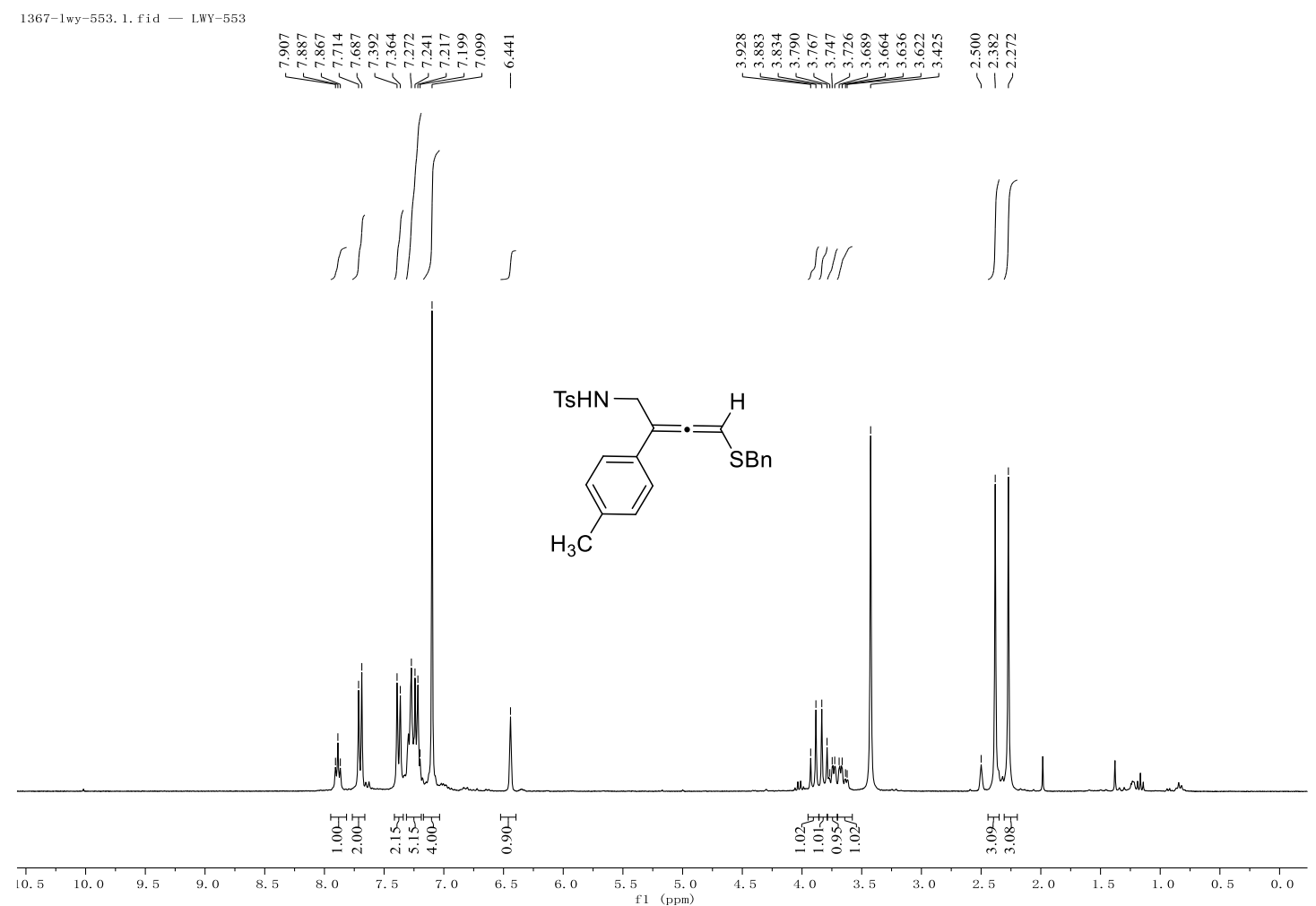

${ }^{13} \mathrm{C}\left\{{ }^{1} \mathrm{H}\right\}$ NMR (101 MHz, DMSO- $\left.d_{6}\right)$ of $\mathbf{5 c}$

\begin{tabular}{|c|c|c|c|c|}
\hline ֻே. & 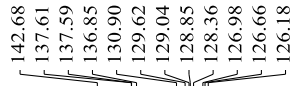 & $\begin{array}{l}\infty \\
0 \\
\infty \\
\infty \\
0 \\
1\end{array}$ & $\begin{array}{l}\stackrel{2}{n} \\
\text { à } \\
i\end{array}$ & 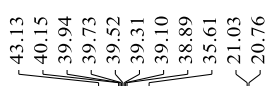 \\
\hline
\end{tabular}

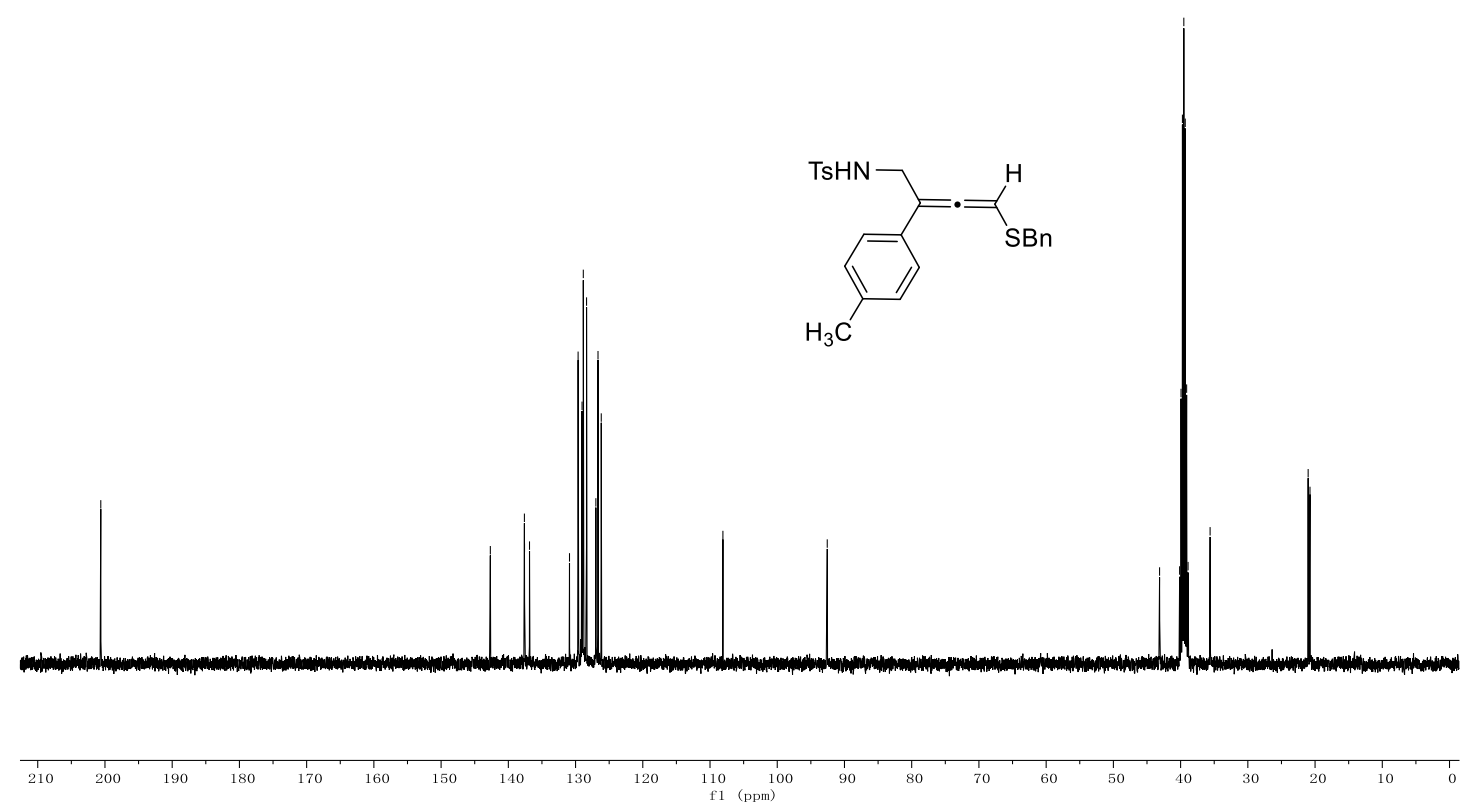


${ }^{1} \mathrm{H}$ NMR (300 MHz, DMSO- $d_{6}$ ) of $\mathbf{5 d}$

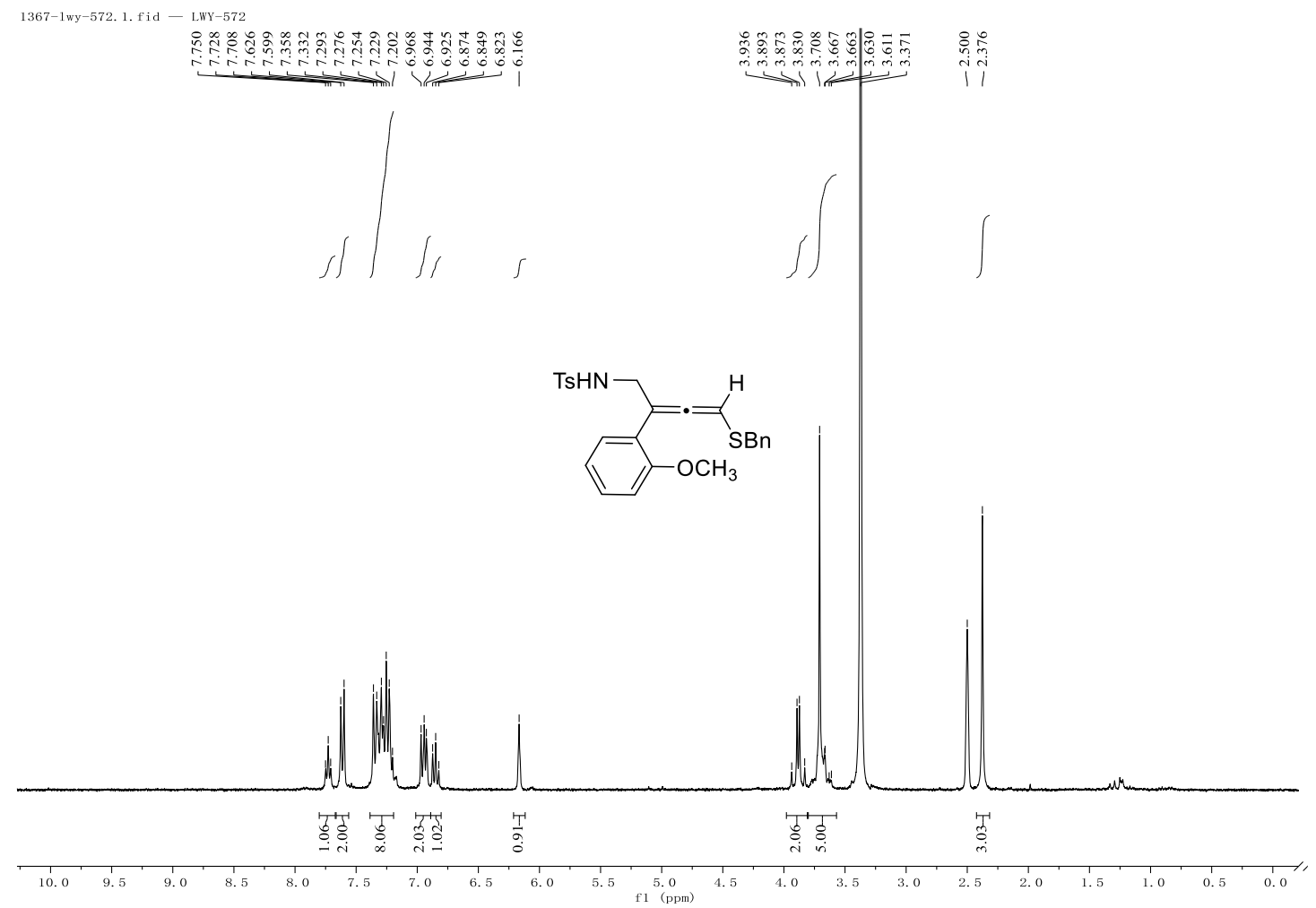

${ }^{13} \mathrm{C}\left\{{ }^{1} \mathrm{H}\right\}$ NMR $\left(101 \mathrm{MHz}\right.$, DMSO- $\left.d_{6}\right)$ of $\mathbf{5 d}$

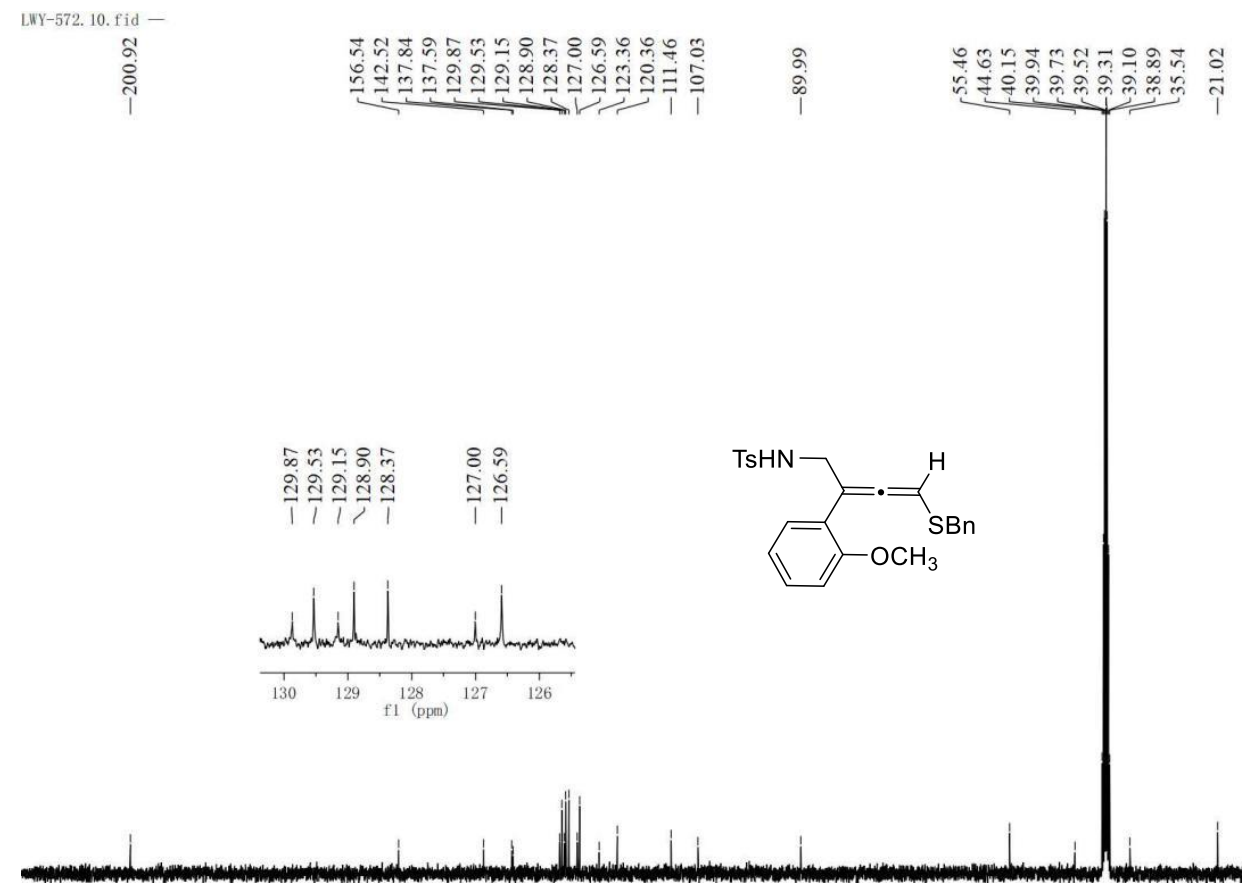

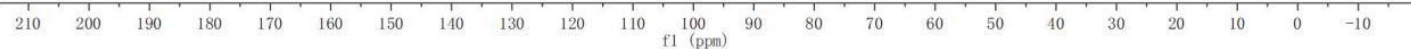


${ }^{1} \mathrm{H}$ NMR (300 MHz, DMSO- $d_{6}$ ) of $\mathbf{5 e}$

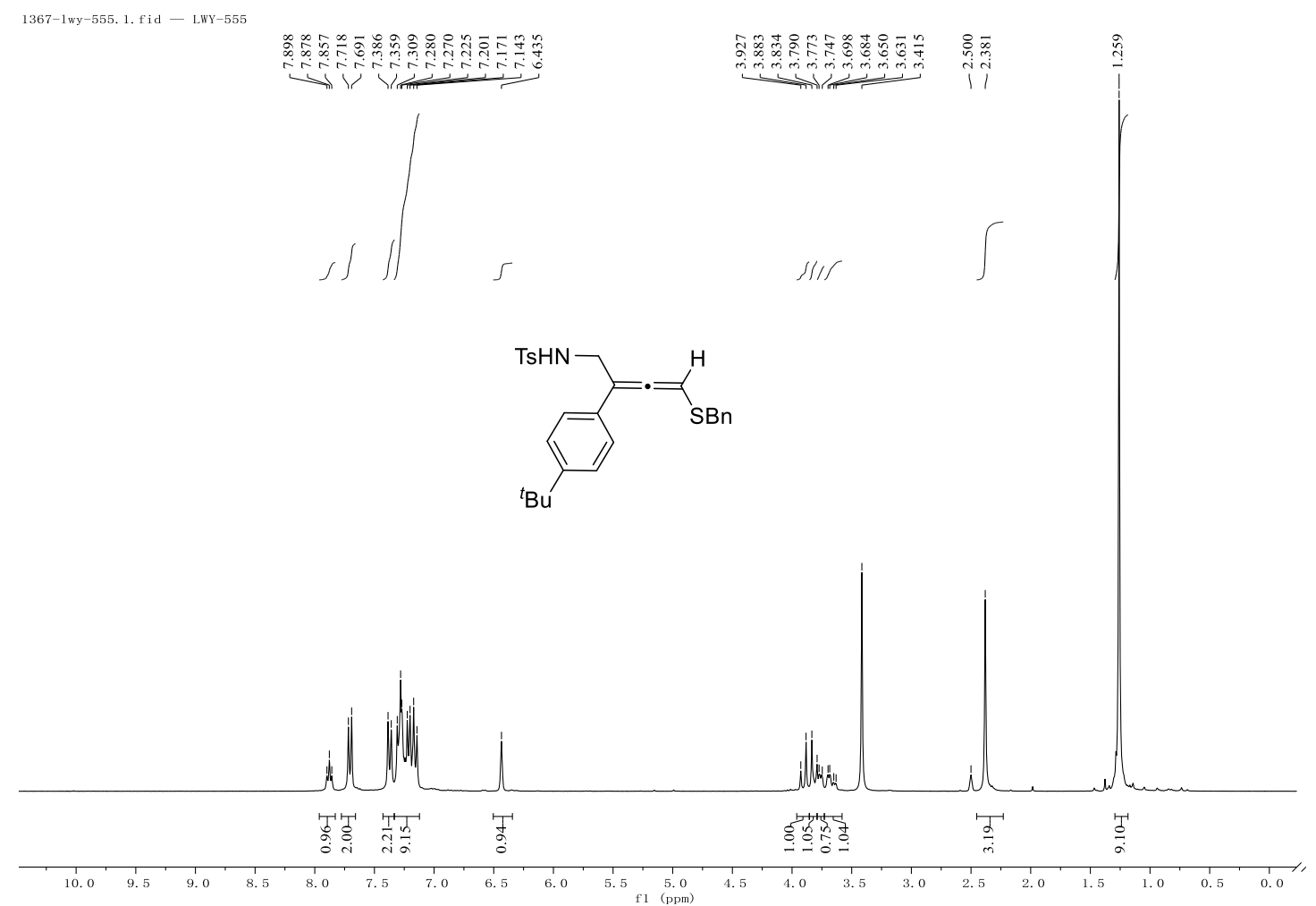

${ }^{13} \mathrm{C}\left\{{ }^{1} \mathrm{H}\right\}$ NMR (101 MHz, DMSO- $\left.d_{6}\right)$ of $\mathbf{5 e}$

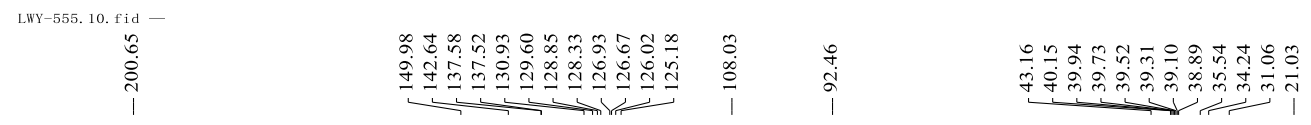

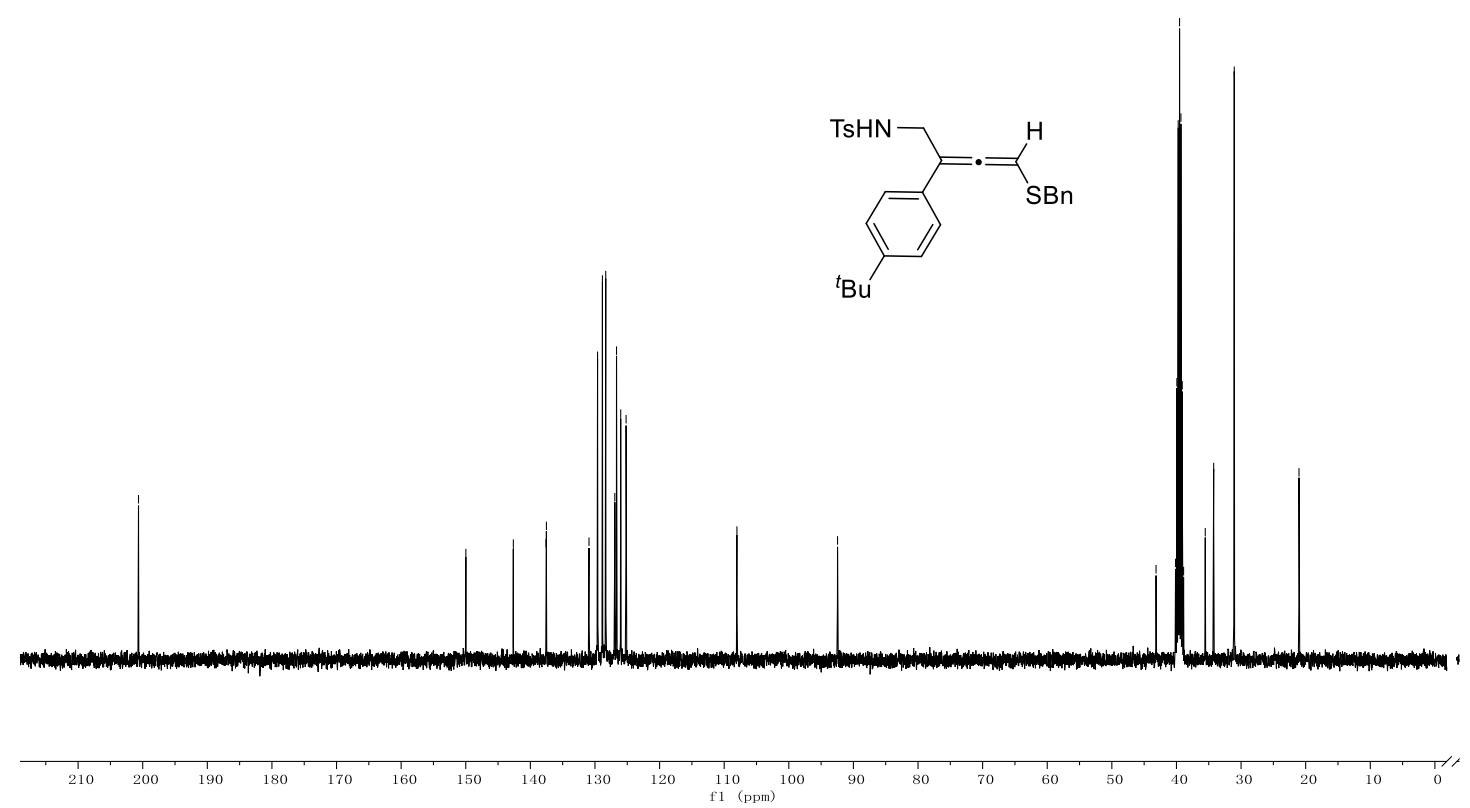


${ }^{1} \mathrm{H}$ NMR (300 MHz, DMSO- $\left.d_{6}\right)$ of $\mathbf{5 f}$

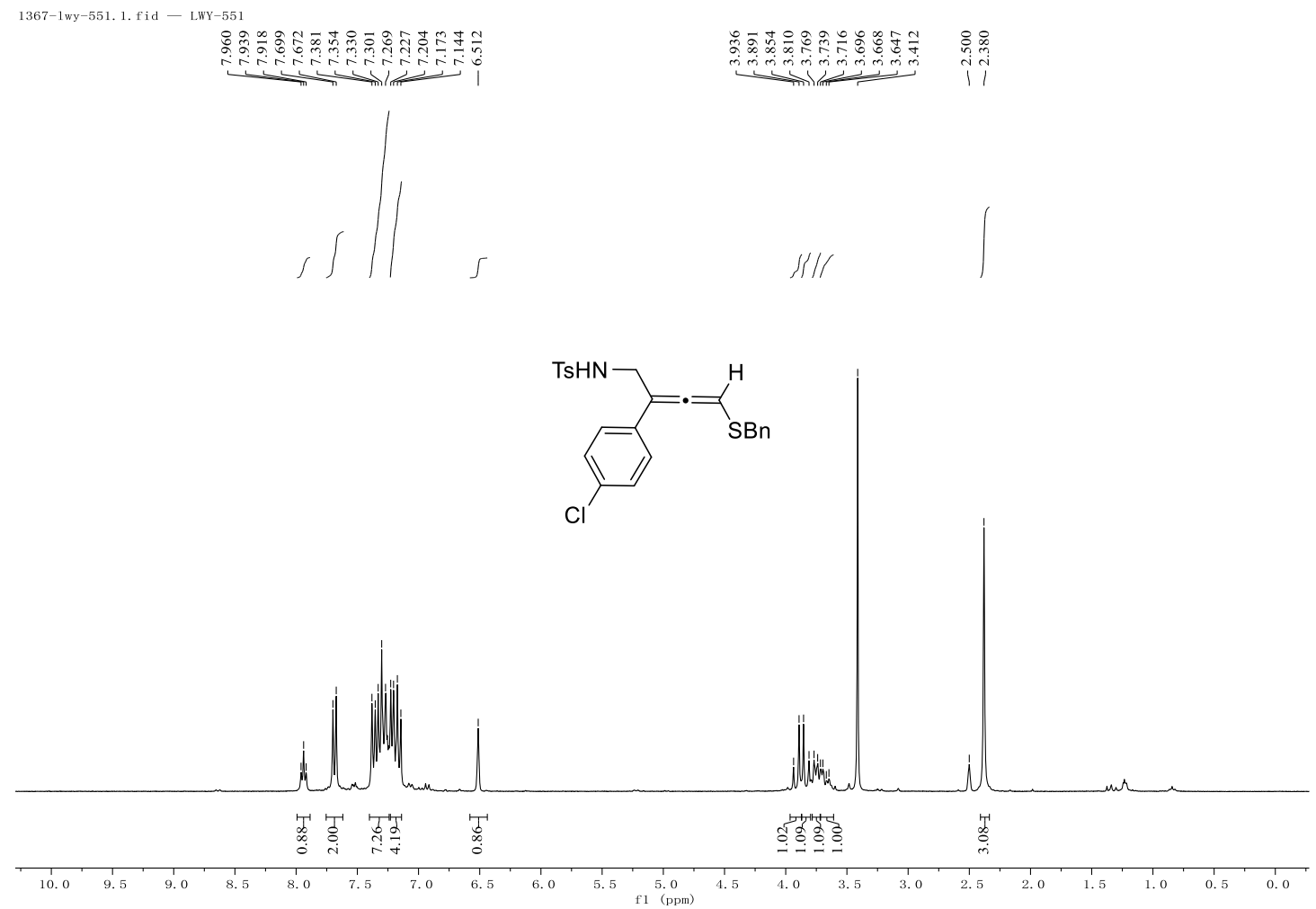

${ }^{13} \mathrm{C}\left\{{ }^{1} \mathrm{H}\right\}$ NMR (101 MHz, DMSO- $\left.d_{6}\right)$ of $\mathbf{5 f}$

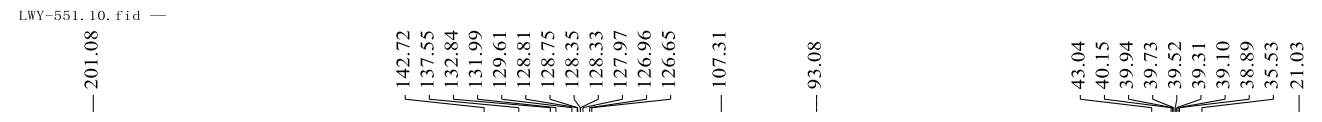
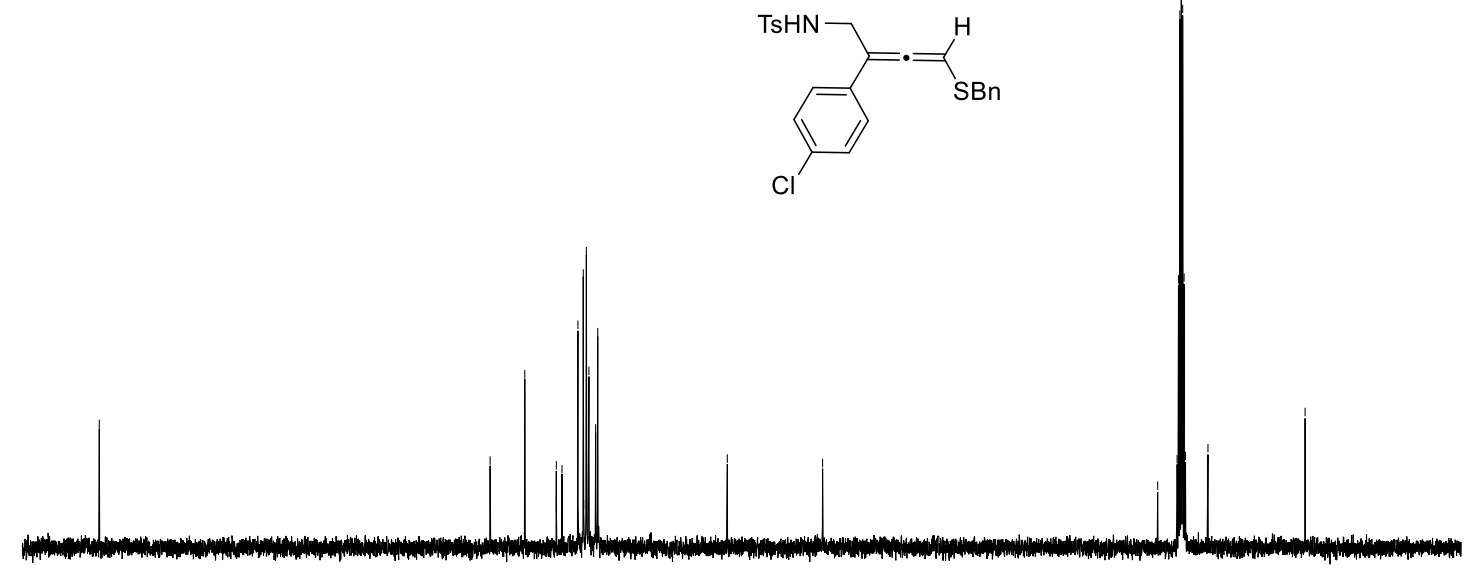

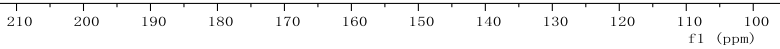


${ }^{1} \mathrm{H}$ NMR (300 MHz, DMSO- $d_{6}$ ) of $\mathbf{5 g}$

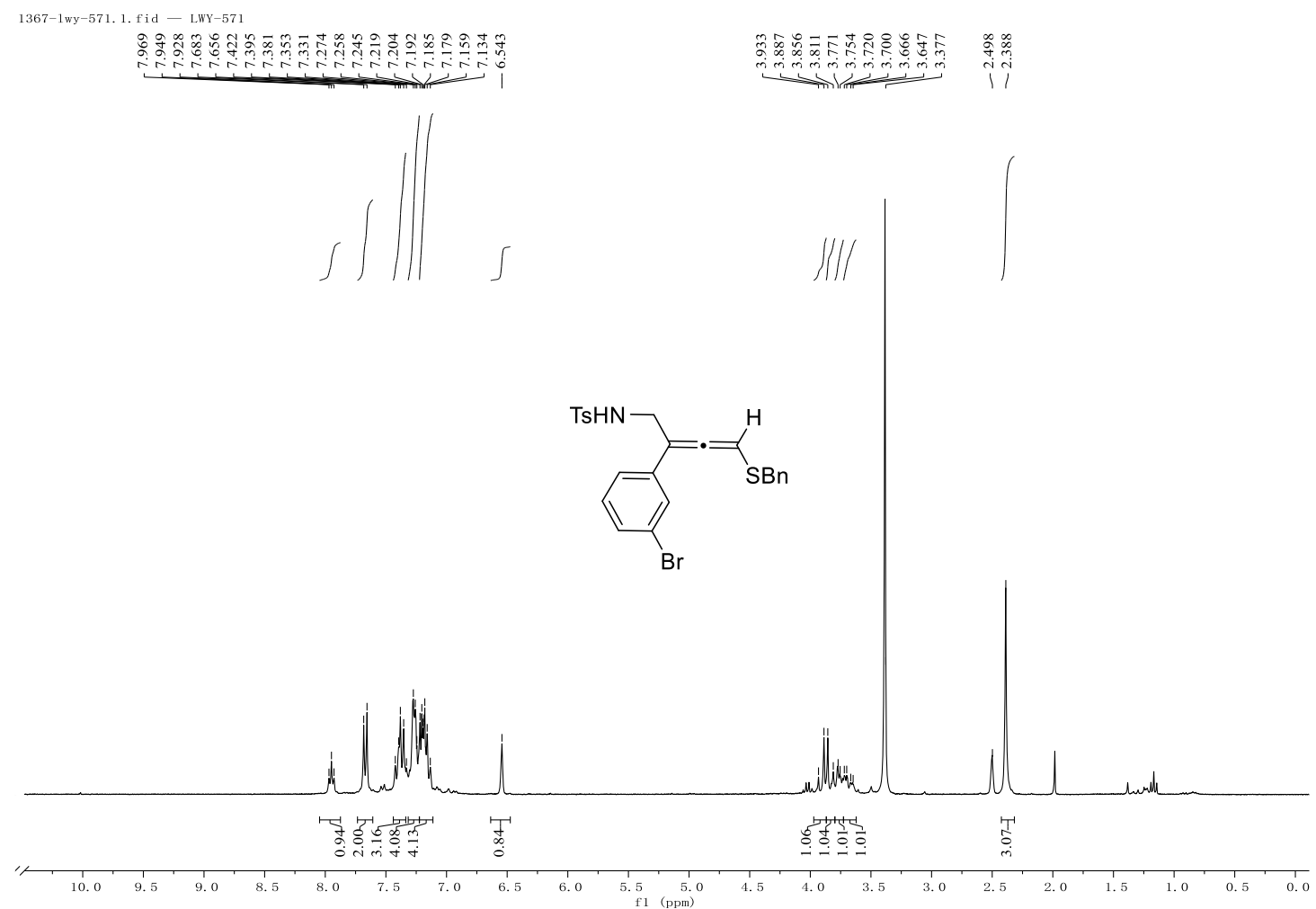

${ }^{13} \mathrm{C}\left\{{ }^{1} \mathrm{H}\right\}$ NMR (101 MHz, DMSO- $\left.d_{6}\right)$ of $\mathbf{5 g}$

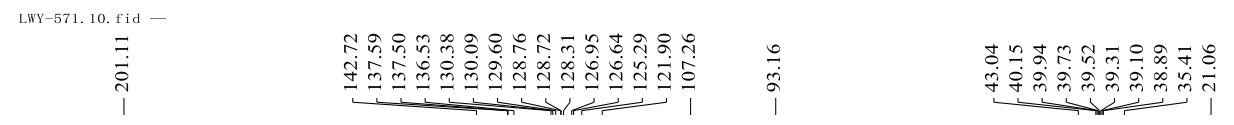
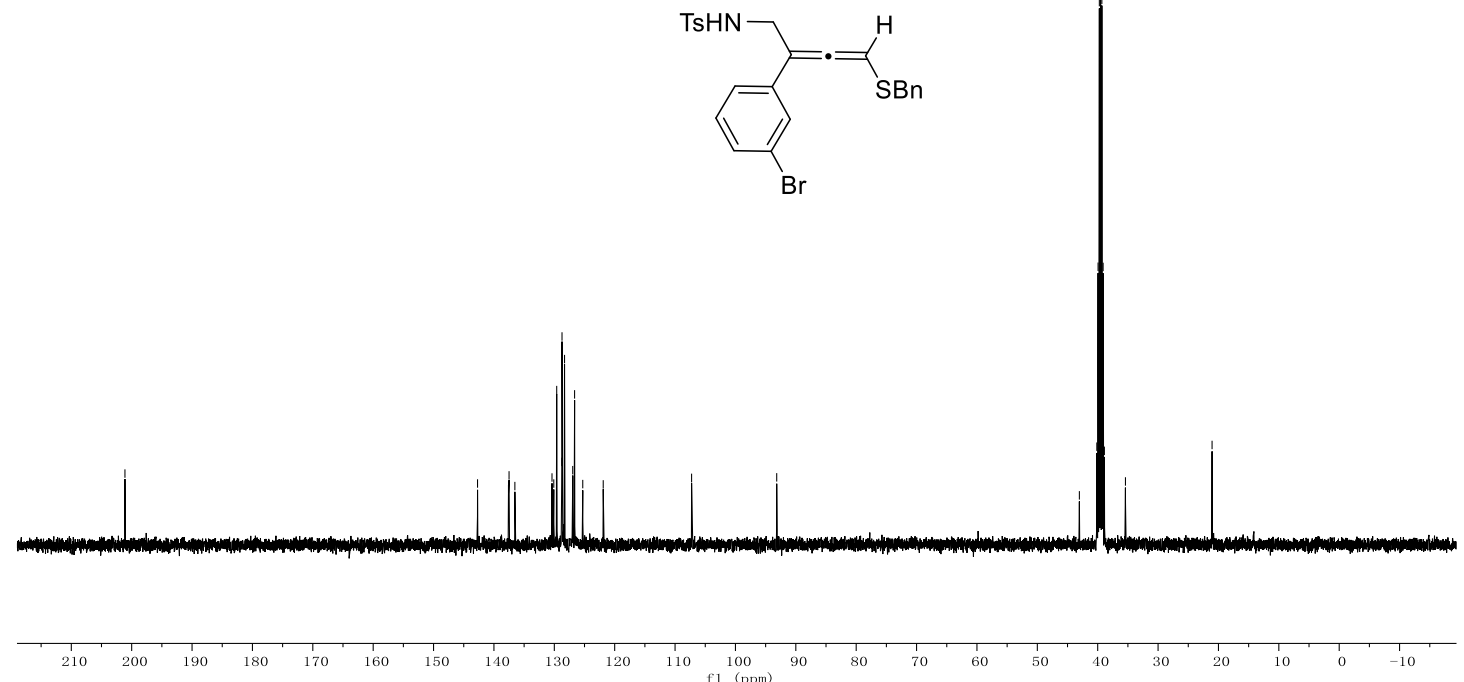
${ }^{1} \mathrm{H}$ NMR (300 MHz, DMSO- $\left.d_{6}\right)$ of $\mathbf{5 h}$

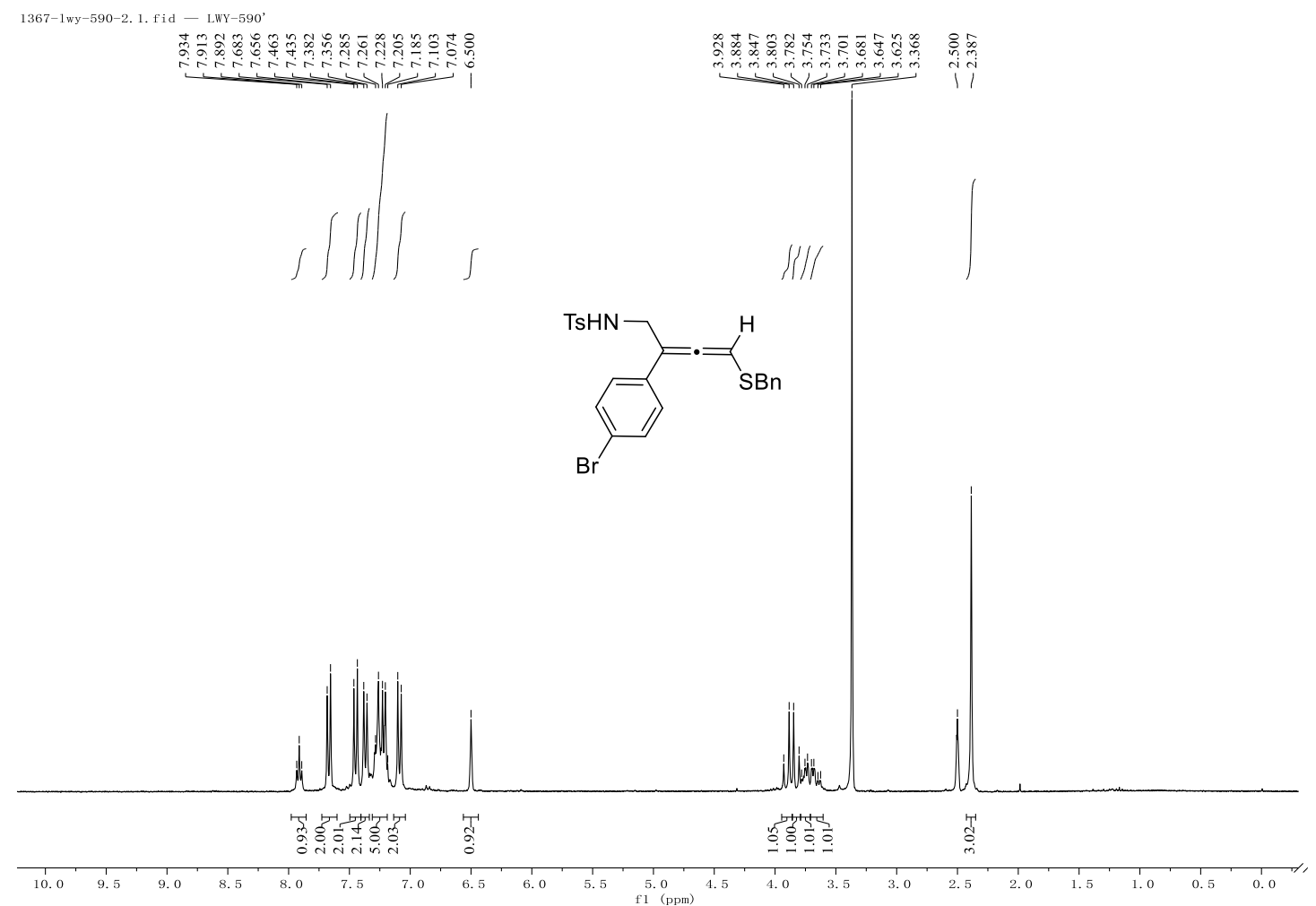

${ }^{13} \mathrm{C}\left\{{ }^{1} \mathrm{H}\right\}$ NMR (101 MHz, DMSO- $\left.d_{6}\right)$ of $\mathbf{5 h}$

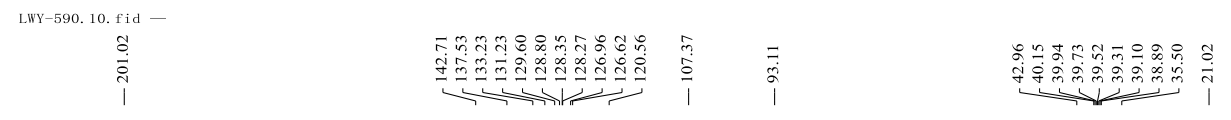
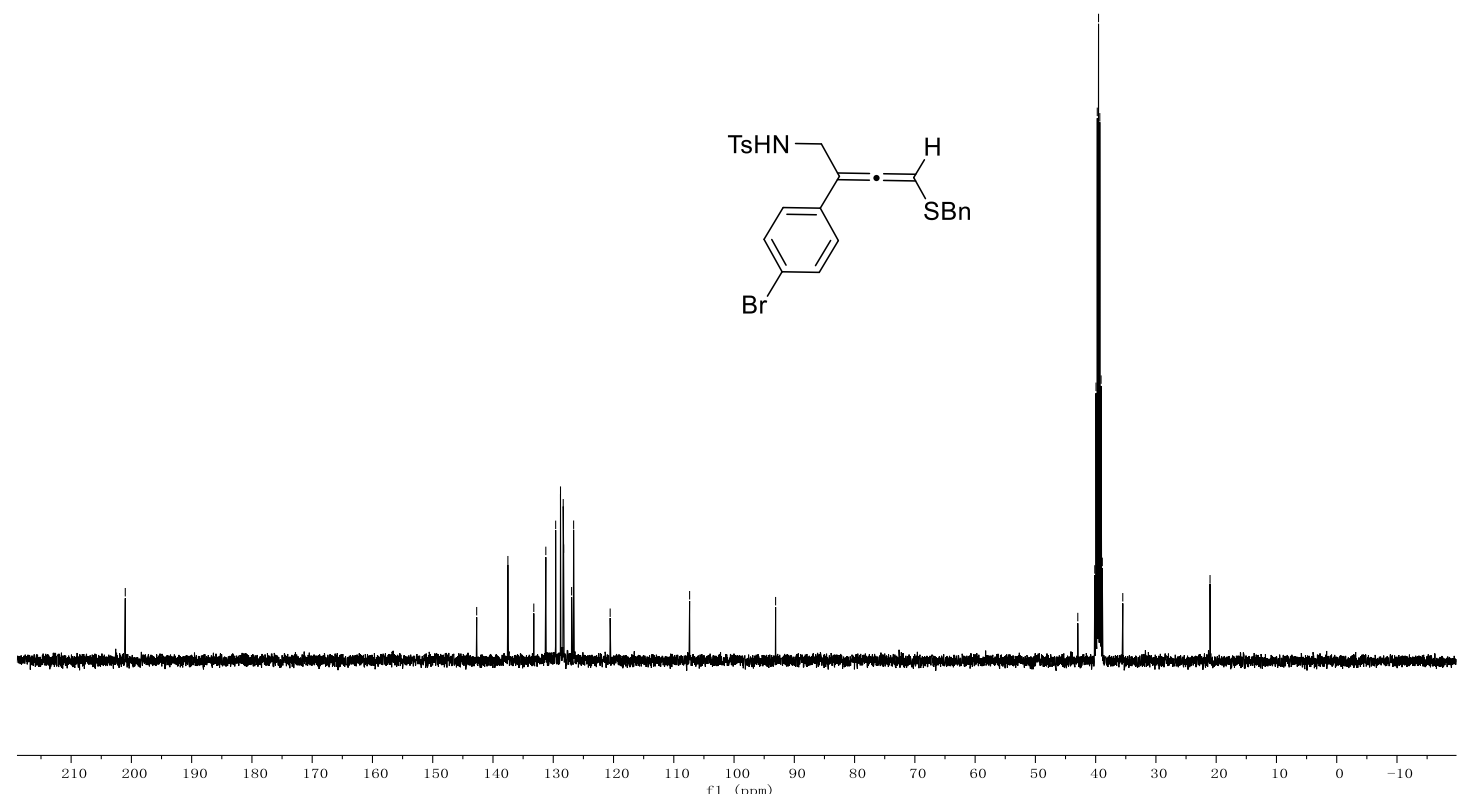
${ }^{1} \mathrm{H}$ NMR (300 MHz, DMSO- $\left.d_{6}\right)$ of $\mathbf{5 i}$

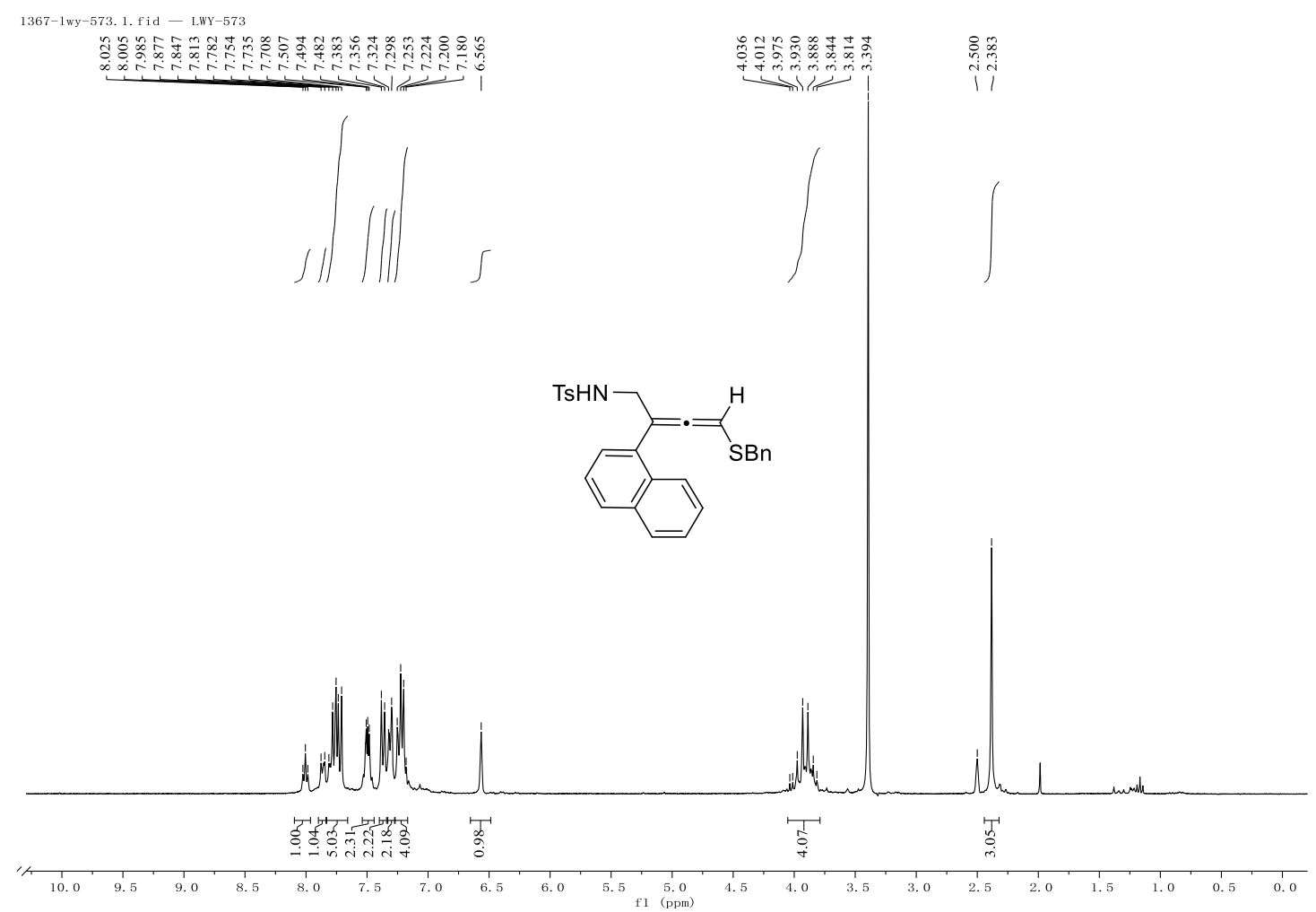

${ }^{13} \mathrm{C}\left\{{ }^{1} \mathrm{H}\right\}$ NMR (101 MHz, DMSO- $\left.d_{6}\right)$ of $\mathbf{5 i}$

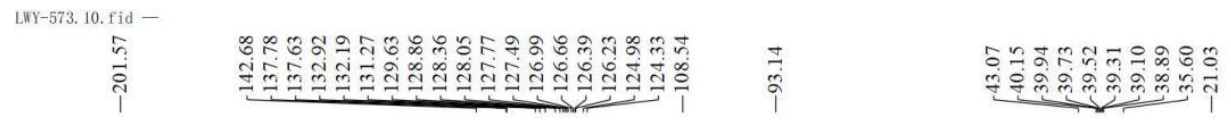

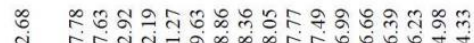

I.

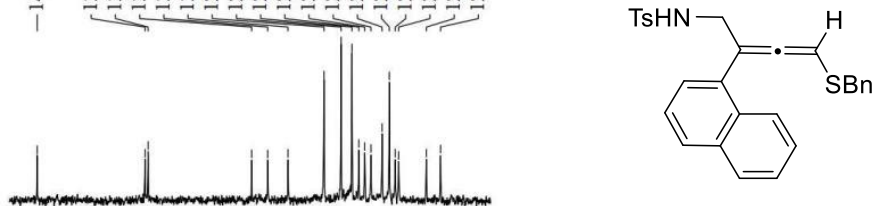

\begin{tabular}{llllllllllllllll}
142 & 140 & 138 & 136 & 134 & 132 & 130 & 128 & 126 & 1 \\
\hline & & & 124 & 124
\end{tabular}
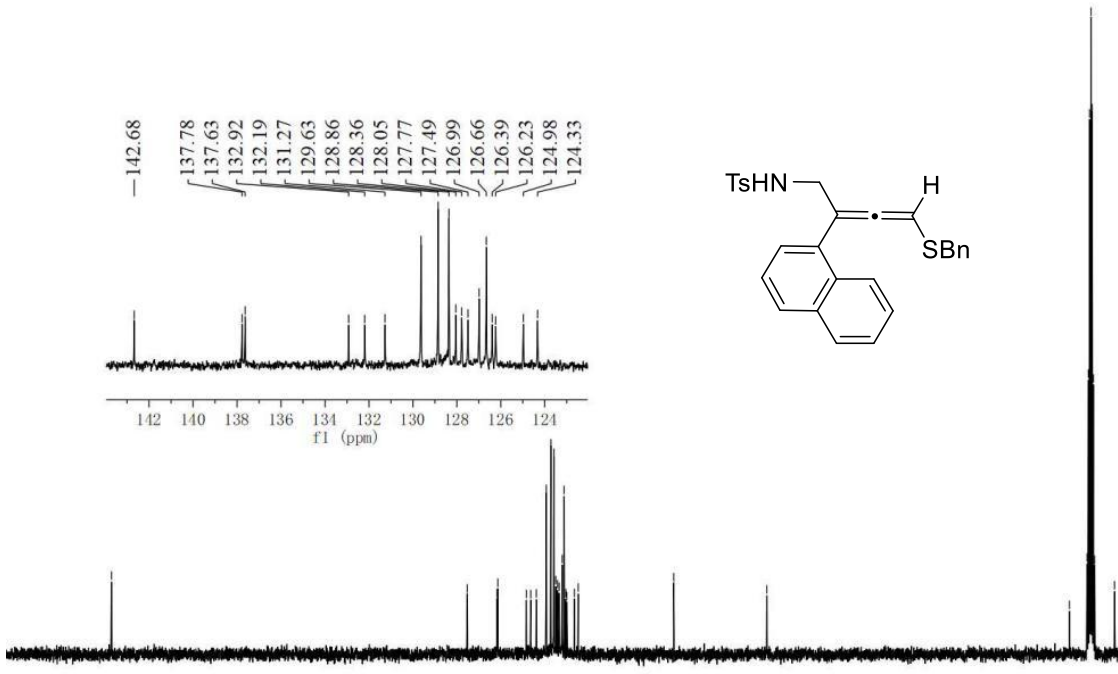

$\begin{array}{lllllllllllllllllllllllllllllll}1 & 200 & 190 & 180 & 170 & 160 & 150 & 140 & 130 & 120 & 110 & 100 & 90 & 80 & 70 & 60 & 50 & 40 & 30 & 20 & 10 & 0 & -10\end{array}$ 
${ }^{1} \mathrm{H}$ NMR (300 MHz, DMSO- $\left.d_{6}\right)$ of $\mathbf{5 j}$

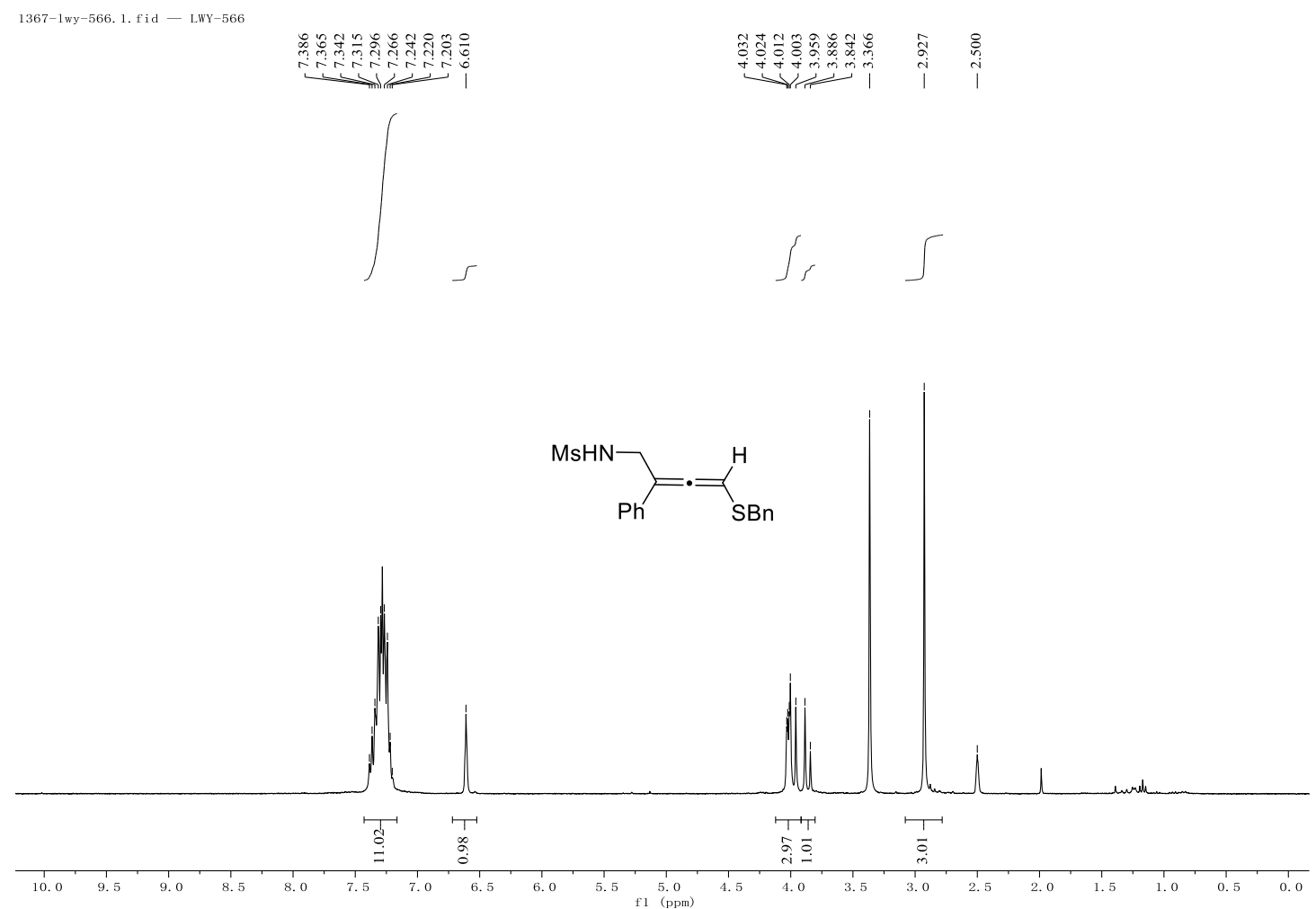

$$
\left.{ }^{13} \mathrm{C}\left\{{ }^{1} \mathrm{H}\right\} \text { NMR (101 MHz, DMSO- } d_{6}\right) \text { of } \mathbf{5 j}
$$

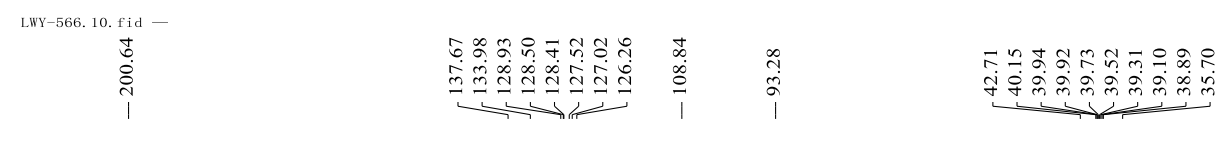
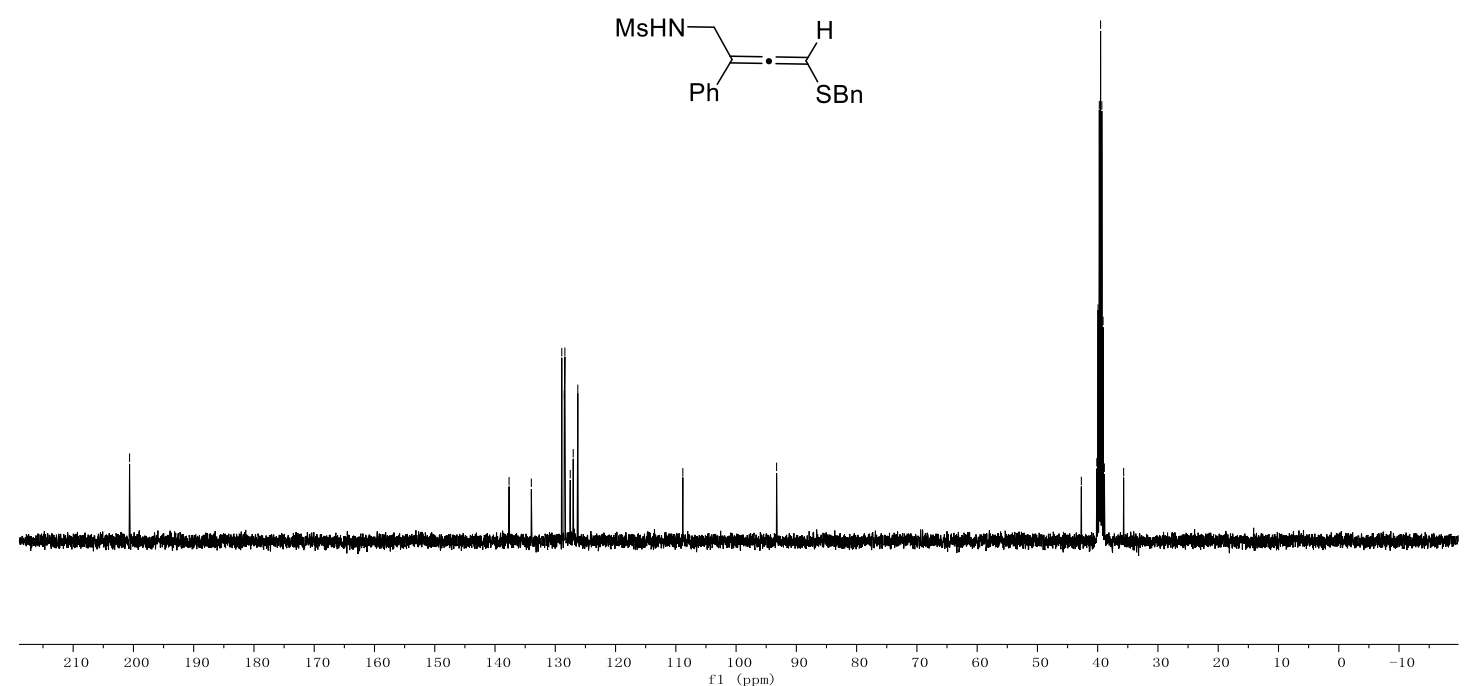
${ }^{1} \mathrm{H}$ NMR (300 MHz, DMSO- $\left.d_{6}\right)$ of $\mathbf{5 k}$

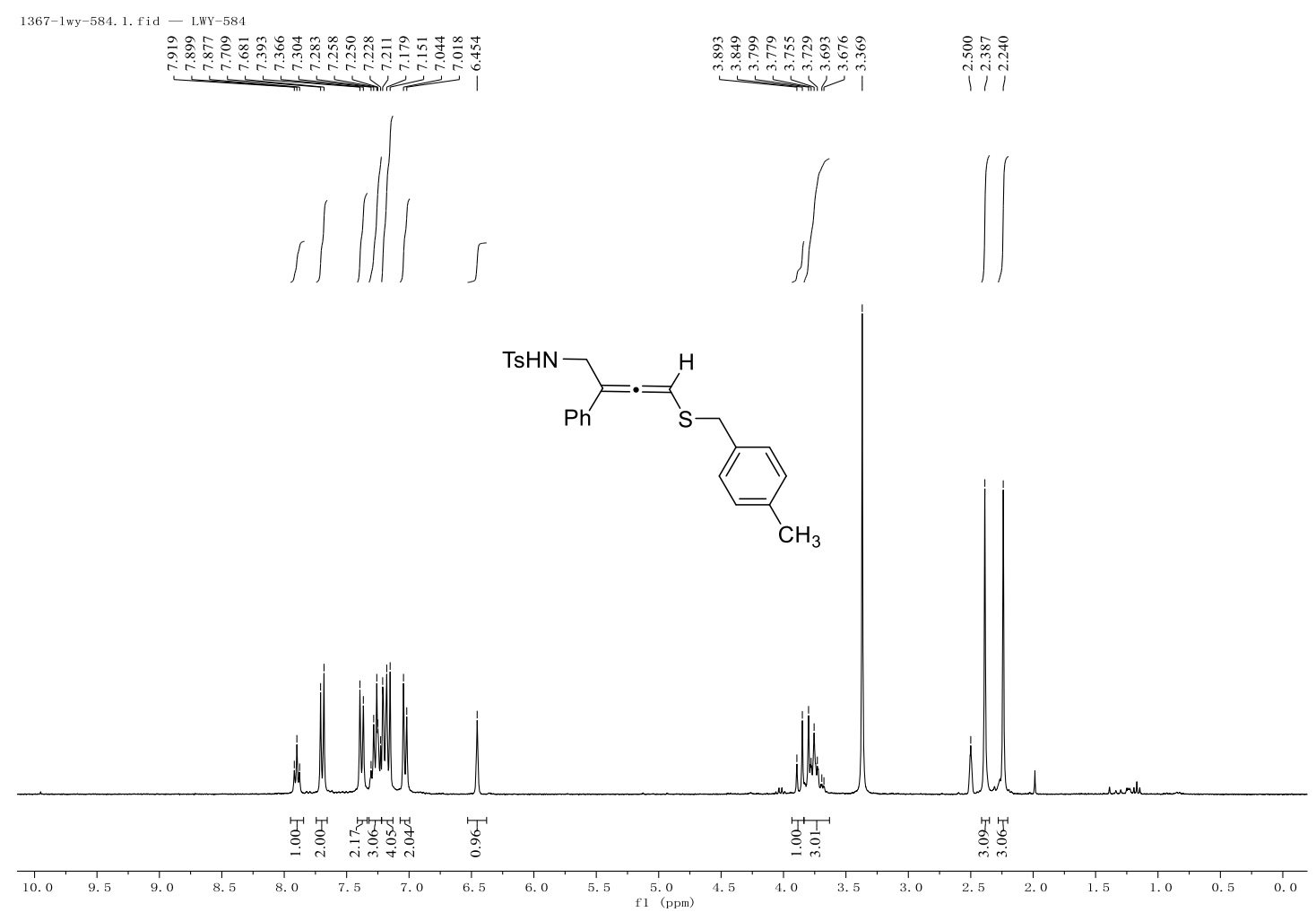

${ }^{13} \mathrm{C}\left\{{ }^{1} \mathrm{H}\right\}$ NMR $\left(151 \mathrm{MHz}\right.$, DMSO- $\left.d_{6}\right)$ of $\mathbf{5 k}$

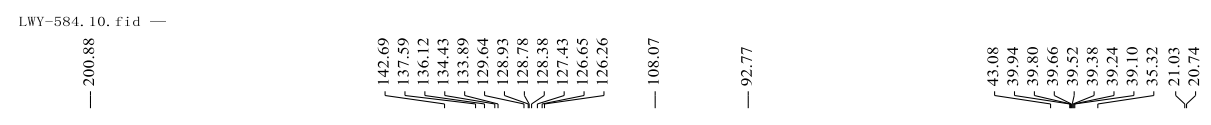

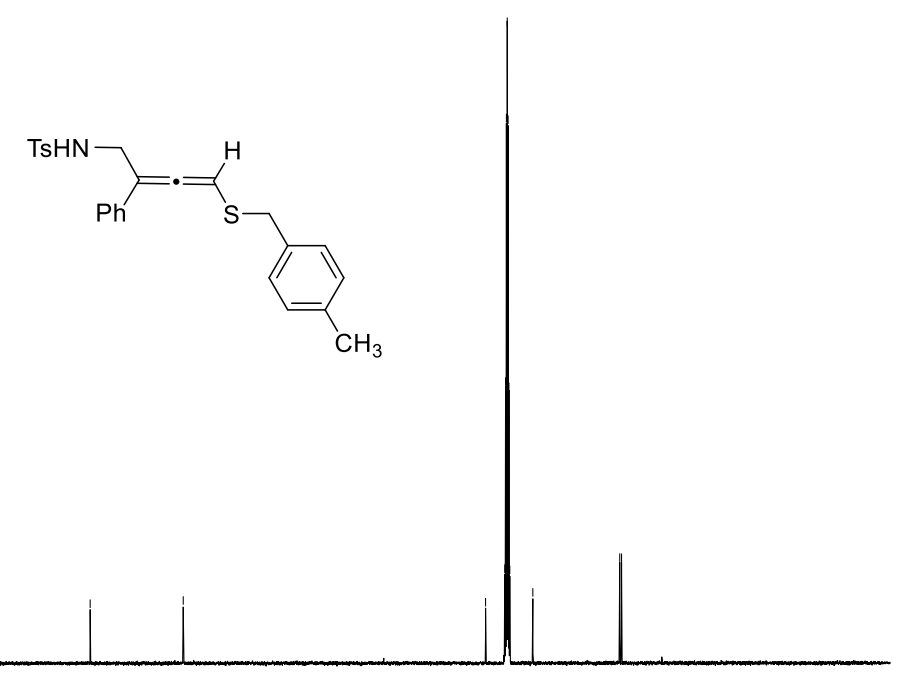

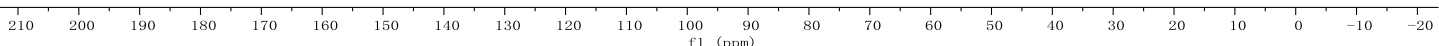


${ }^{1} \mathrm{H}$ NMR (300 MHz, DMSO- $\left.d_{6}\right)$ of $5 \mathbf{I}$

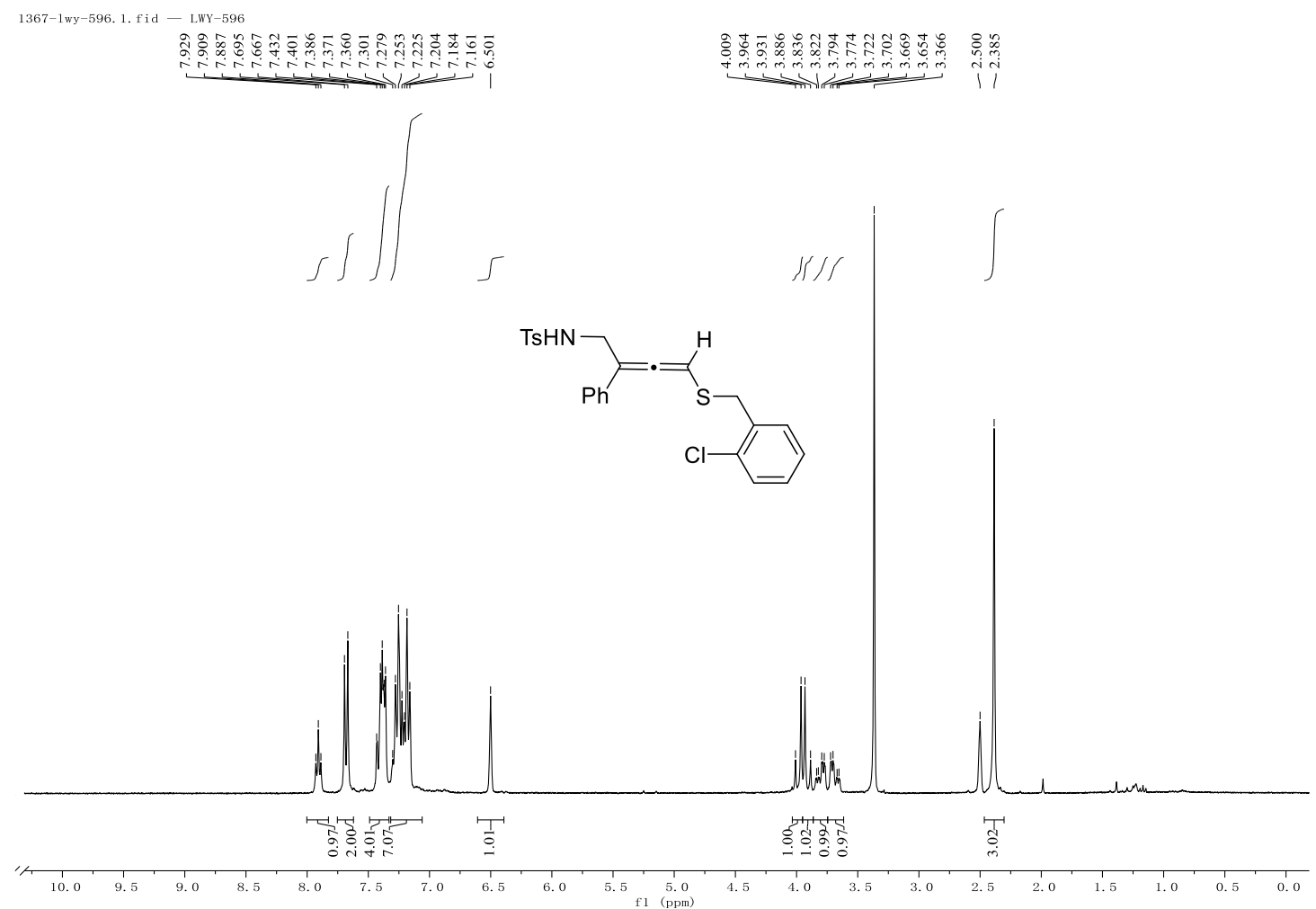

${ }^{13} \mathrm{C}\left\{{ }^{1} \mathrm{H}\right\}$ NMR (151 MHz, DMSO- $\left.d_{6}\right)$ of $\mathbf{5 l}$

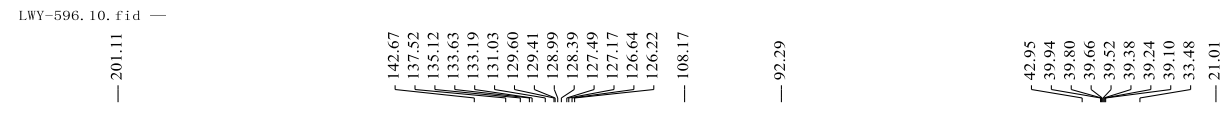
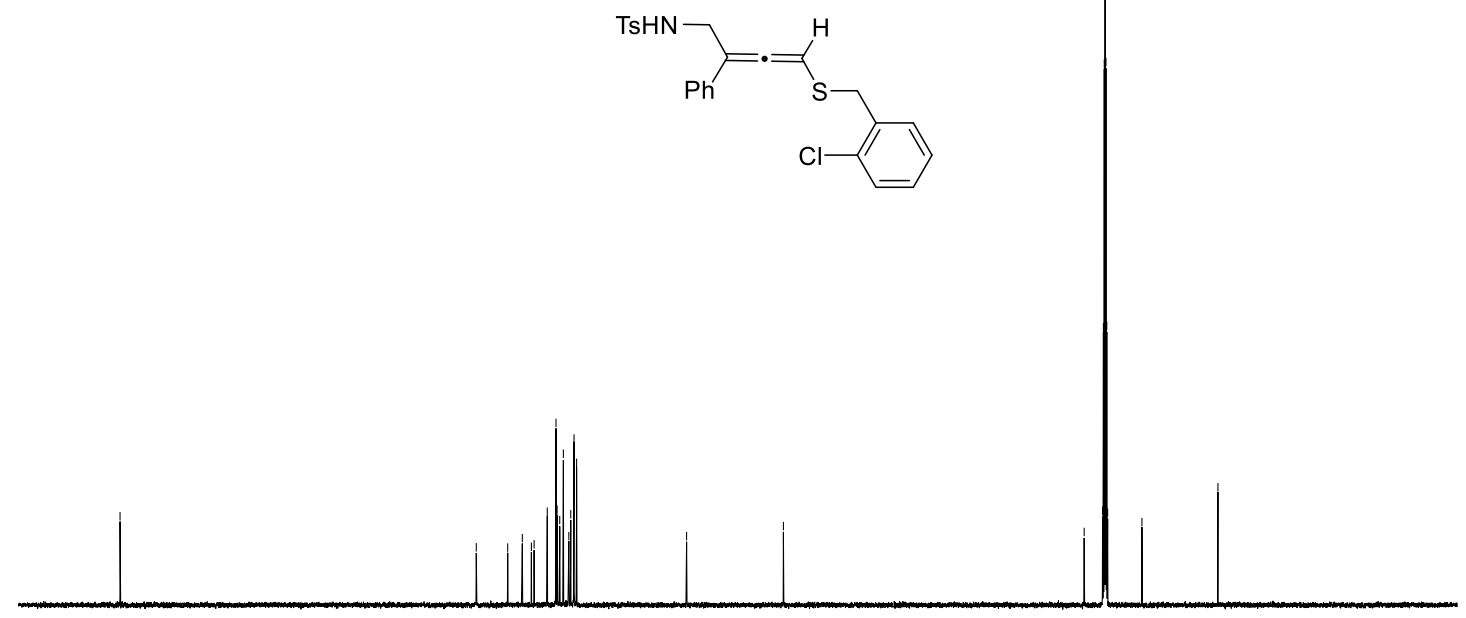

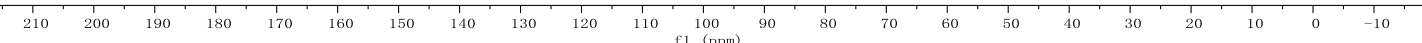


${ }^{1} \mathrm{H}$ NMR (300 MHz, DMSO- $d_{6}$ ) of 6

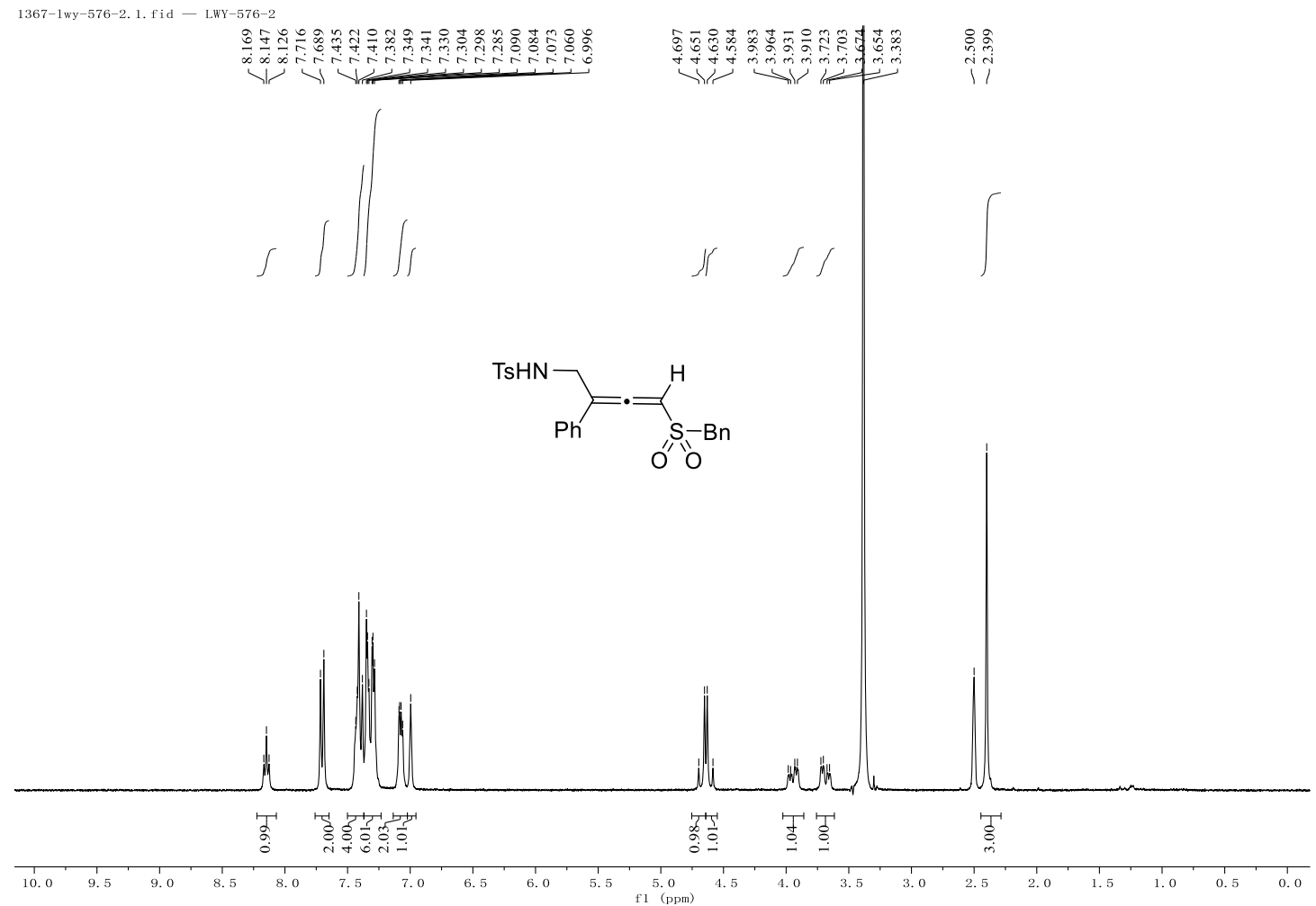

${ }^{13} \mathrm{C}\left\{{ }^{1} \mathrm{H}\right\}$ NMR $\left(151 \mathrm{MHz}\right.$, DMSO- $\left.d_{6}\right)$ of $\mathbf{6}$

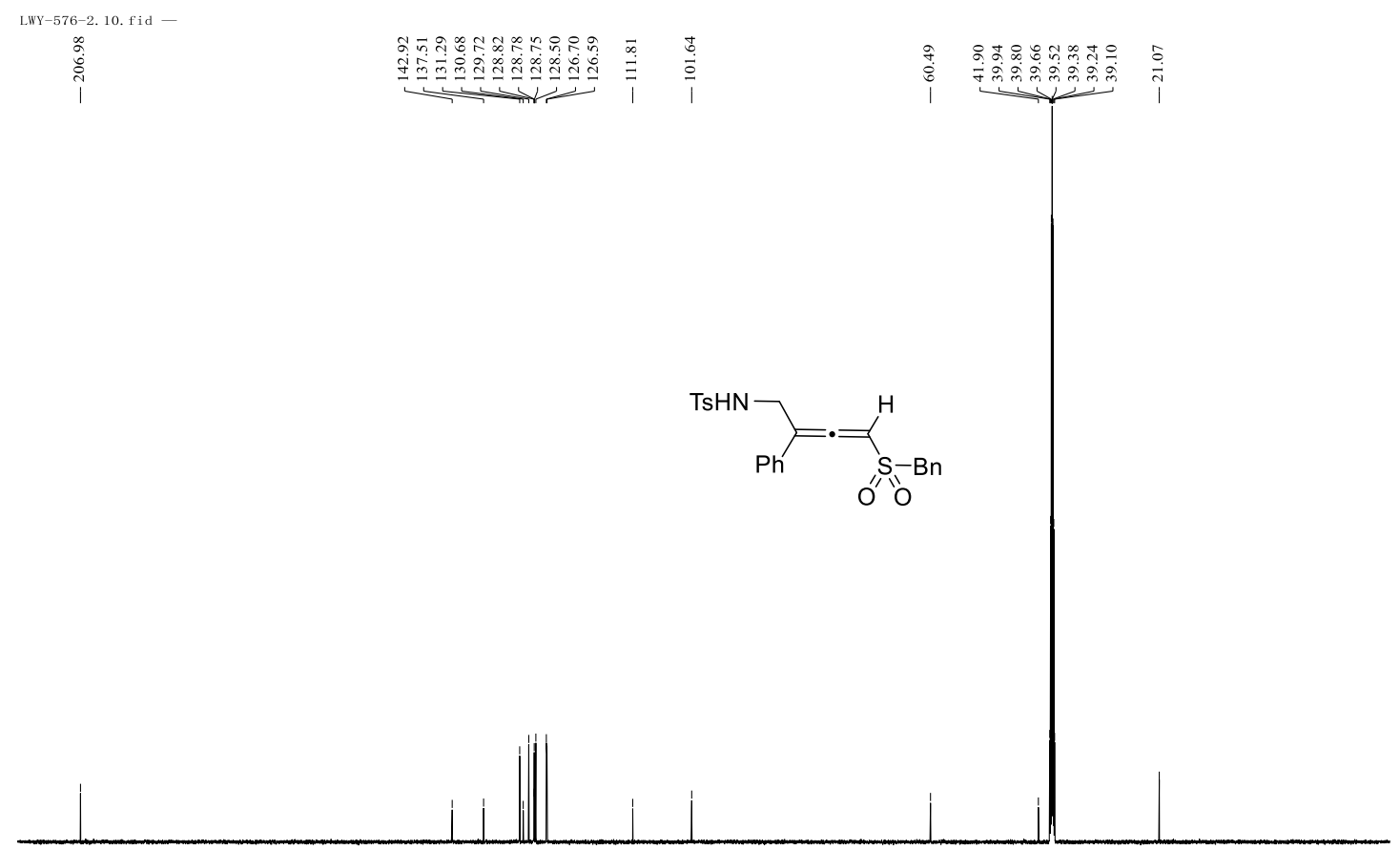

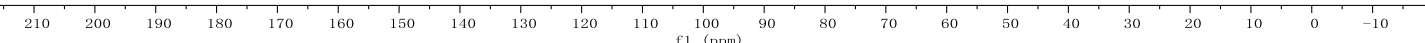


${ }^{1} \mathrm{H}$ NMR $\left(300 \mathrm{MHz}\right.$, DMSO- $\left.d_{6}\right)$ of 7

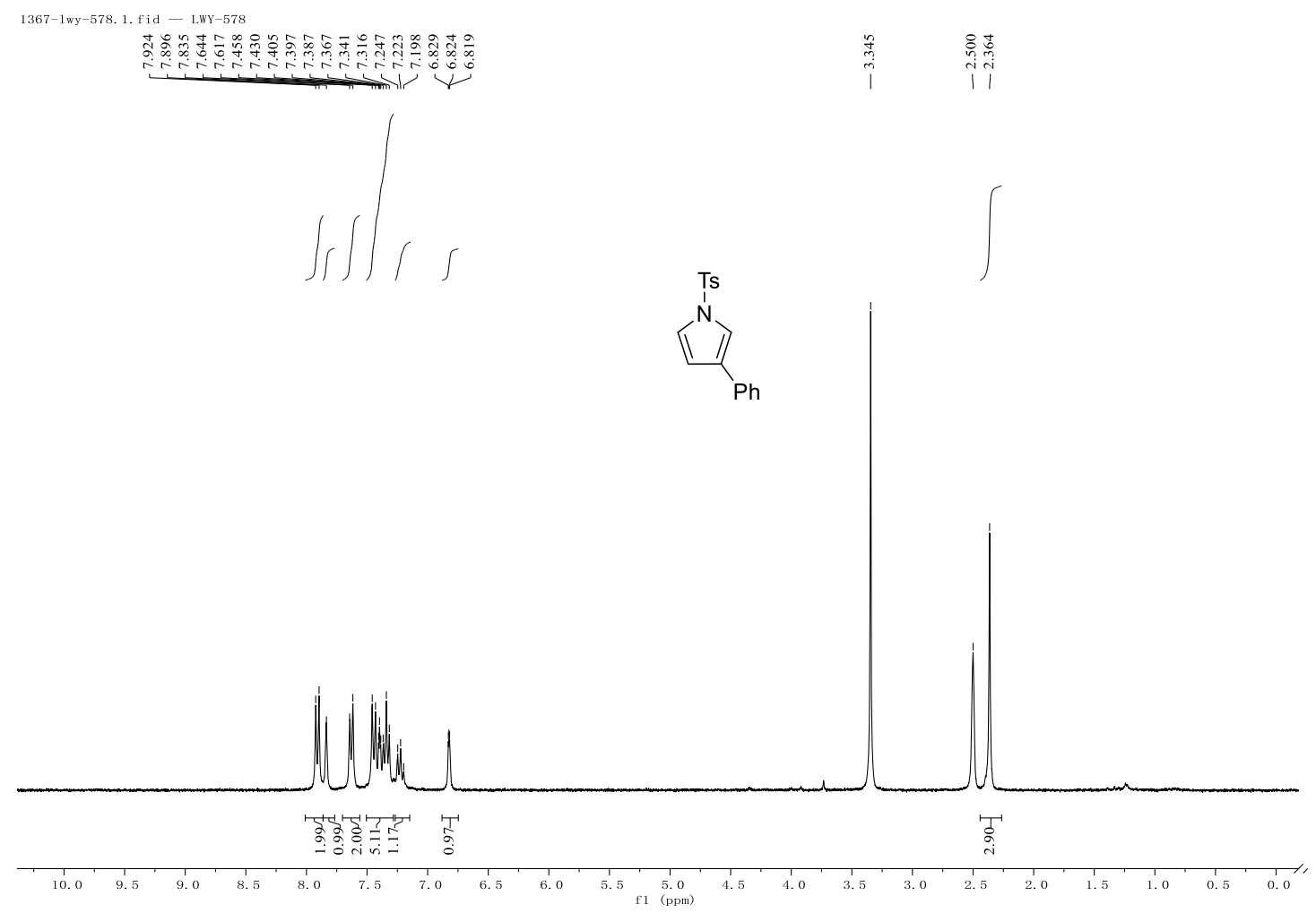

${ }^{13} \mathrm{C}\left\{{ }^{1} \mathrm{H}\right\}$ NMR (151 MHz, DMSO- $\left.d_{6}\right)$ of 7

LWY-578. 10. fid -

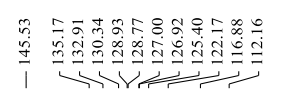

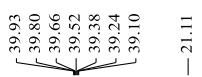

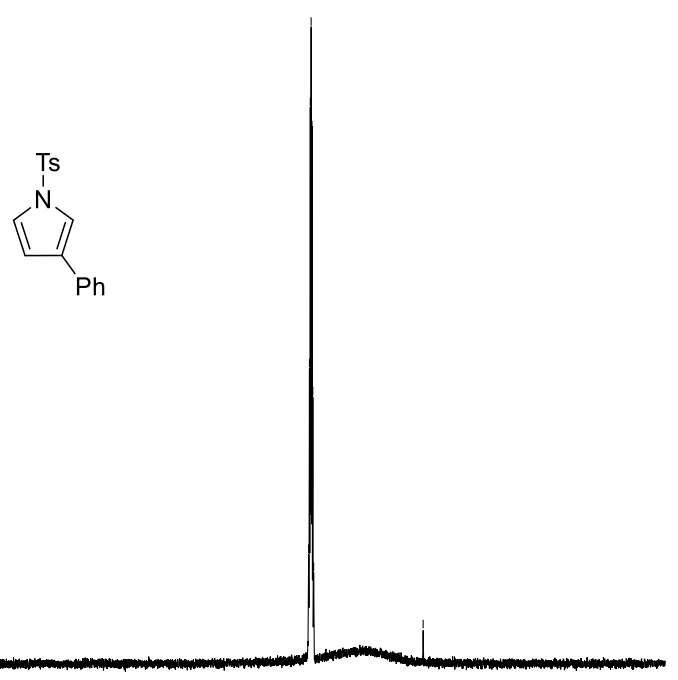

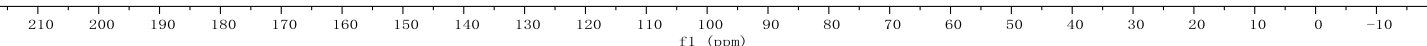


${ }^{1} \mathrm{H}$ NMR (300 MHz, DMSO- $d_{6}$ ) of 8

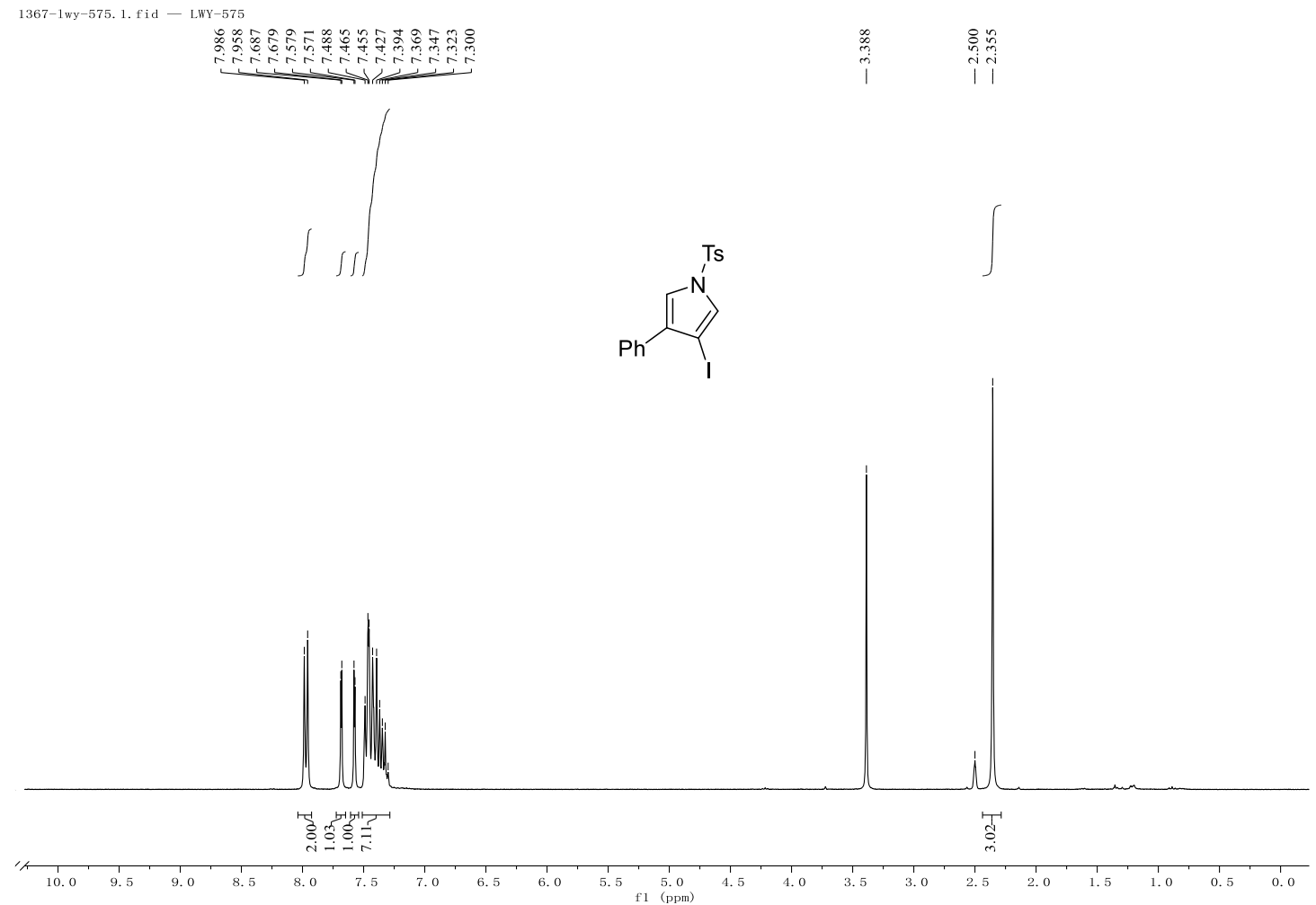

${ }^{13} \mathrm{C}\left\{{ }^{1} \mathrm{H}\right\}$ NMR (151 MHz, DMSO- $\left.d_{6}\right)$ of 8

LWY-575. 10. fid
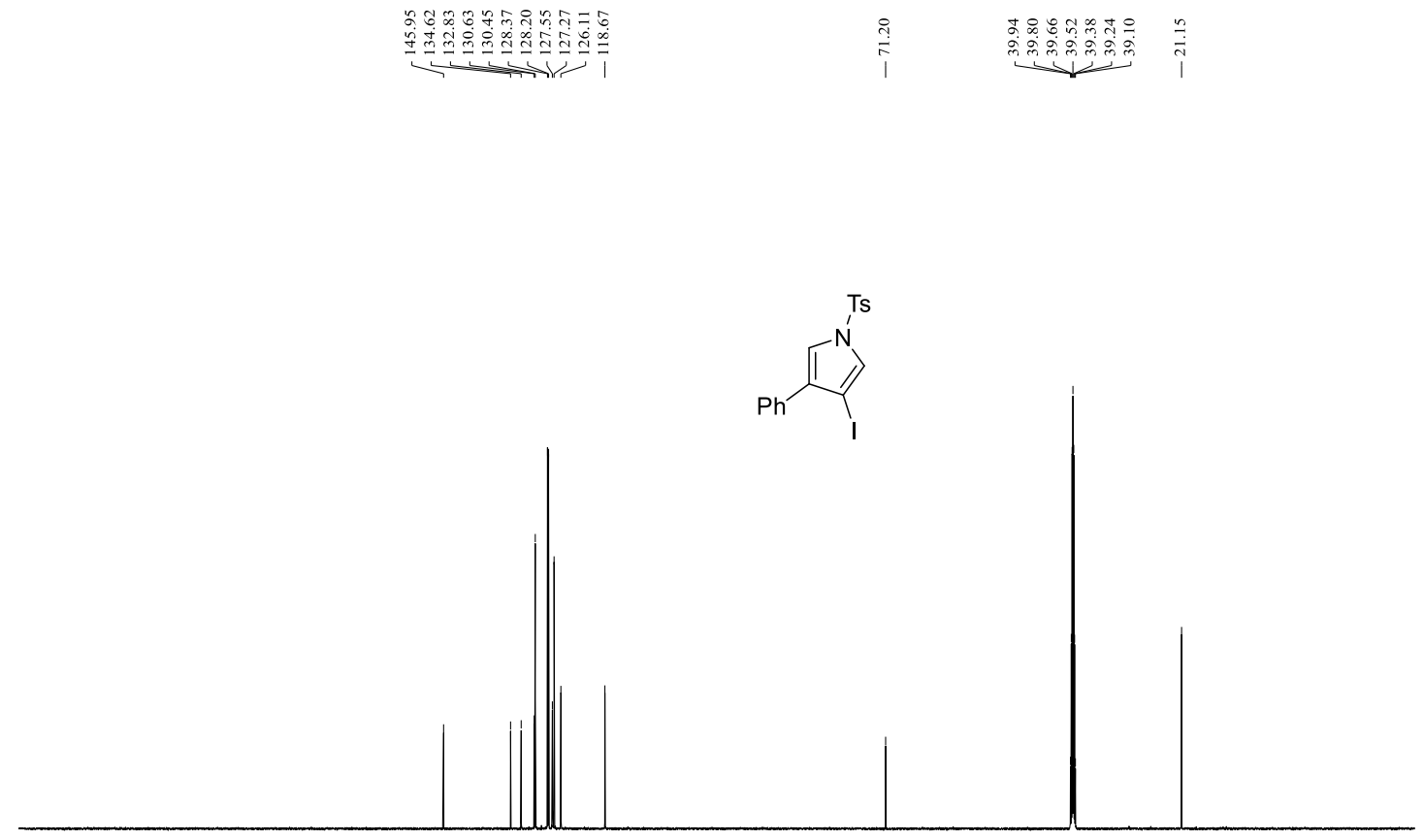

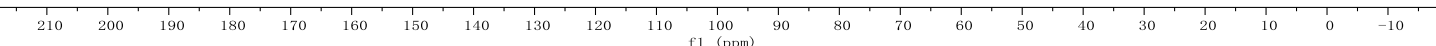


${ }^{1} \mathrm{H}$ NMR (300 MHz, DMSO- $\left.d_{6}\right)$ of 9

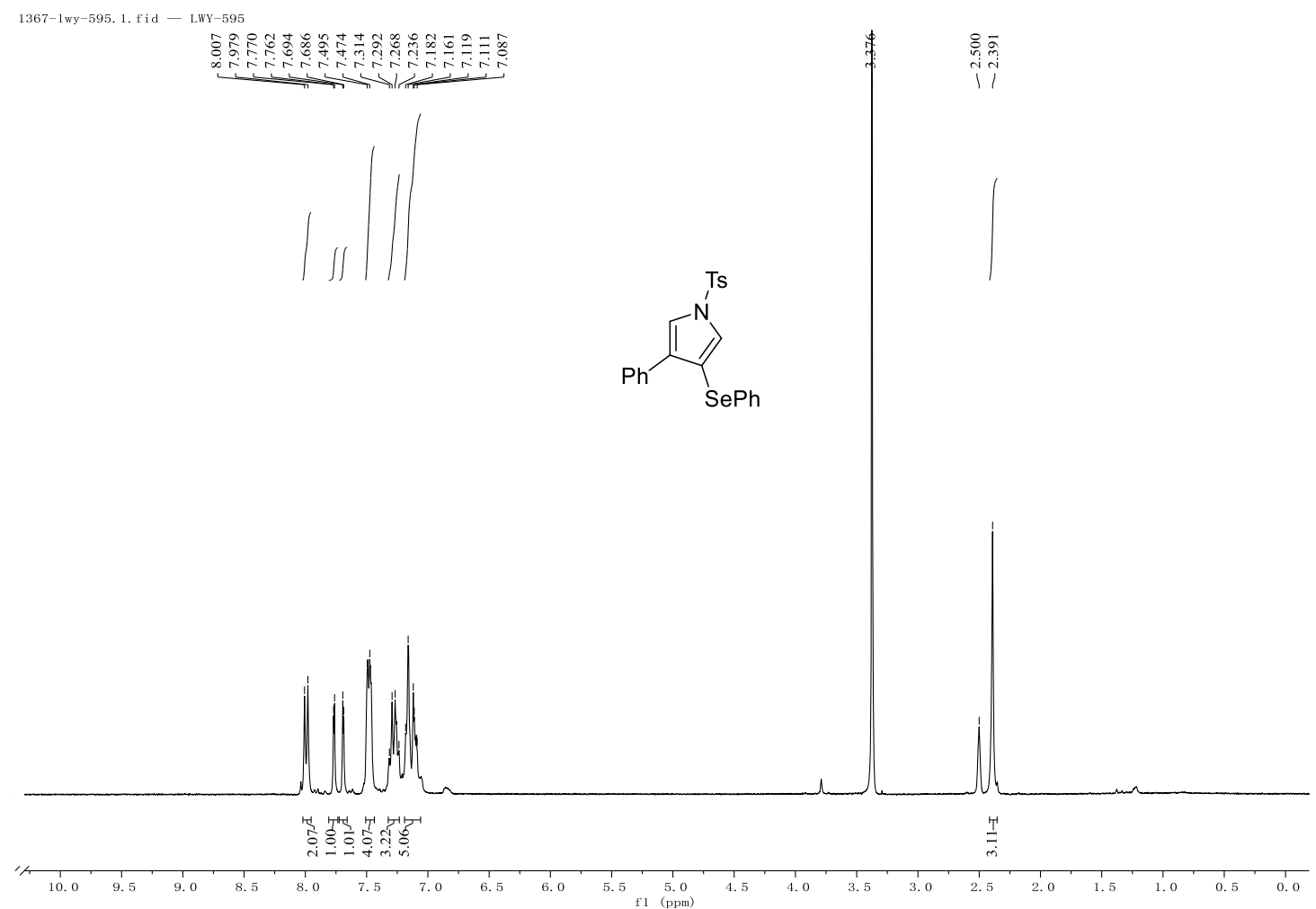

${ }^{13} \mathrm{C}\left\{{ }^{1} \mathrm{H}\right\}$ NMR (101 MHz, DMSO- $\left.d_{6}\right)$ of 9

LWY-595. 10. fid -
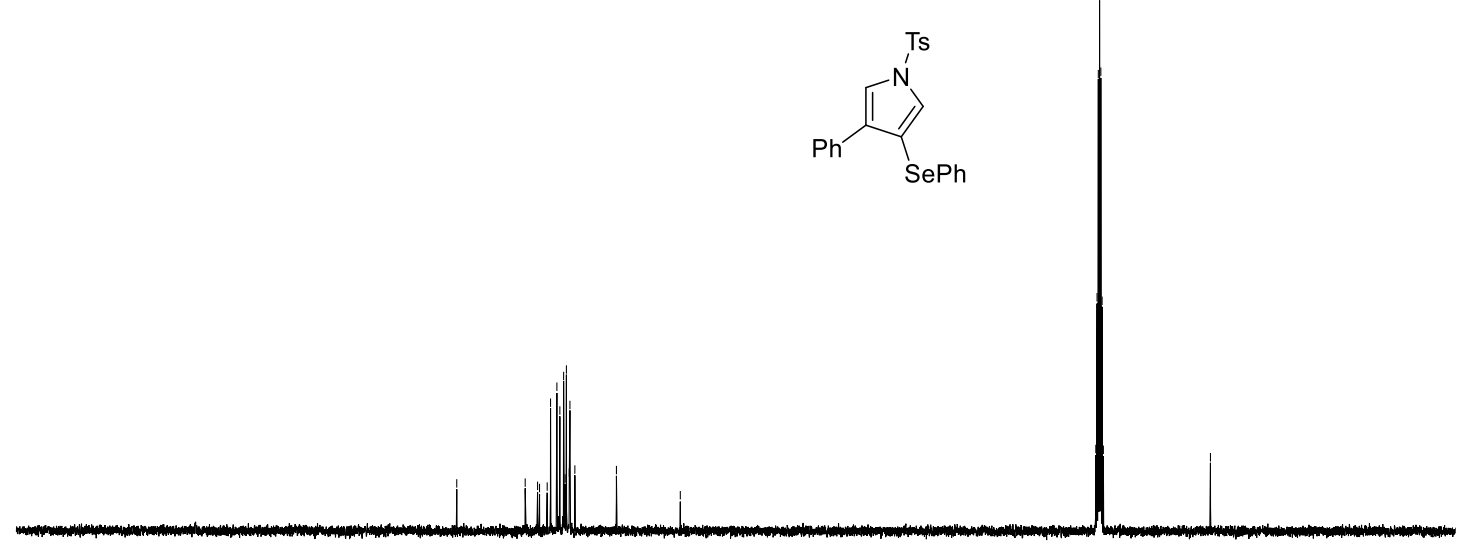